\title{
Modelling and simulation of surface morphology driven by ion bombardment
}

\author{
Dissertation \\ zur Erlangung des Doktorgrades \\ der Mathematisch-Naturwissenschaftlichen Fakultäten \\ der Georg-August-Universität zu Göttingen
}

vorgelegt von

Emmanuel Oluwole Yewande

aus Ibadan/Nigeria

Göttingen 2006 
D7

Referent: PD. Dr. Alexander K. Hartmann

Korreferent: Prof. Dr. Hans Hofsäß

Tag der mündlichen Prüfung: 02.05.2006 


\section{Abstract}

Non-equilibrum surfaces, at nanometer length scales, externally driven via bombardment with energetic particles are known to exhibit well ordered patterns with a variety of applications in nano-technology. These patterns emerge at time scales on the order of minutes. Continuum theory has been quite successful in giving a general picture of the processes that interplay to give the observed patterns, as well as how such competition might determine the properties of the nanostructures. However, continuum theoretical descriptions are ideal only in the asymptotic limit. The only other theoretical alternative, which happens to be more suitable for the characteristic lengthand time-scales of pattern formation, is Monte Carlo simulation.

In this thesis, surface morphology is studied using discrete solid-on-solid Monte Carlo models of sputtering and surface diffusion. The simulations are perfomed in the context of the continuum theories and experiments. In agreement with the experiments, the ripples coarsen with time and the ripple velocity exhibits a power-law behaviour with the ripple wavelength, in addition, the exponent was found to depend on the simulation temperature, which suggests future experimental studies of flux dependence. Moreover, a detailed exploration of possible topographies, for different sputtering conditions, corresponding to different materials, was performed. And different surface topographies e.g. holes, ripples, and dots, were found at oblique incidence, without sample rotation. With sample rotation no new topography was found, its only role being to destroy any inherent anisotropy in the system. 


\section{Dedication}

To my wife Taiwo, and children: Seun, Tobi, and Tife. 


\section{Acknowledgements}

First and foremost I would like to thank my thesis advisor PD. Dr. Alexander Hartmann for invaluable advice not only as pertains to this work, but numerical modelling and computation in general. I would like to thank Prof. Dr. Reiner Kree, Prof. Dr. Hans Hofsäß, and Dr. Rodolfo Cuerno (Madrid) for very helpful and fruitful discussions. In addition, I would like to thank Prof. Dr. Annette Zippelius, Prof. Dr. Helmut Grubmüller, Prof. Dr. Christian Griesinger, PD. Dr. Peter Müller, PD. Dr. Martin Weigt, Dr. Timo Aspelmeier, Henning Löwe, Olaf Herbst, Jens-Timo Neumann, Sebastian Fuchs, Garrit Jentsch, and all other colleagues. Special thanks to Dorothea Witt. I would also like to thank Waheed Adeagbo, Kunle, and Funmi Adegoke.

It has been a great pleasure to study and live in Göttingen, and I would like to thank all members of the Theorie Institut in this regard. In particular, I would like to thank all members (past and present) of our junior research group Complex ground states of disordered systems, headed by Alex: Dr. Bernd Burghardt, Wolgang Barthel, Carlo Amoruso, Alejandro Morales Gallardo, Stefan Wolfsheimer, Martin Zumsande, and Magnus Jungsbluth. Also, my special thanks to Frauen Gisela Mesecke, Gabriele Schubert, Katrin Glormann, and Bärbel Lütge-Hampe.

This work was funded by the German research foundation, the Deutsche forchungsgemeinscaft (DFG), within the sonderforchungsbereich (SFB) 602: Complex Structures in Con densed Matter from Atomic to Mesoscopic Scales. 


\section{List of Abbreviations}

AES: Auger electron spectroscopy

AFM: stomic force microscope

AKPZ: anisotropic KPZ (see KPZ)

BCA: binary collision approximation

BH: Bradley-Harper

CB: Cuerno-Barabási

COSIPO: computer simulation in polycrystals

$D$ : spatial dimension

DT: Das Sarma-Tamborenea

EW: Edwards-Wilkinson

FIBM: focussed ion beam microscope

HTEM: high-resolution transmission electron microscope

KMC: kinetic Monte Carlo

KPZ: Kardar-Parisi-Zhang

KS: Kuramoto-Sivashinsky

MC: Monte Carlo

MD: Molecular dynamics

PDE: partial differential equation

PSSR: profiles from simultaneous sputtering and rotation

QD: quantum dot

RHEED: reflection high-energy electron diffraction

SEM: scanning electron microscope

SIMS: secondary ion mass spectroscopy

SRIM: stopping and range of ions in matter

STM: scanning tunneling microscope

WV: Wolf-Villain 


\section{Contents}

Title i

Abstract iii

Dedication iv

Acknowledgements $\quad$ v

List of Abbreviations $\quad$ vi

Table of Contents viii

List of Figures $\quad x$

$\begin{array}{lll}1 & \text { Introduction } & 1\end{array}$

2 Experiments 4

2.1 Amorphous and SemiconductorMaterials . . . . . . . . . . . 4

2.2 Metallic Materials . . . . . . . . . . . . . . . . . 13

2.3 Summarv . . . . . . . . . . . . . . . . . . . 14

3 Continuum Theorv 16

3.1 Introduction . . . . . . . . . . . . . . . . . . . . . . . . 16

3.2 Scaling of Interface Fluctuations . . . . . . . . . . . . . . 17

3.3 Effect of Each Component . . . . . . . . . . . . . . . . . . . . 19

3.4 Linear Theorv . . . . . . . . . . . . . . . . . . . . . . 21

3.4.1 Bradlev-Harper Theorv . . . . . . . . . . . . . 21

3.4.2 Theorv of Makeev, Cuerno, and Barabási . . . . . . . . 23

3.5 Non-linear Theorv . . . . . . . . . . . . . . . 25

3.5.1 The Kardar-Parisi-Zhang Equation . . . . . . . . . . 26

3.5.2 The Kuramoto-Sivashinskv Equation . . . . . . . . . . 27

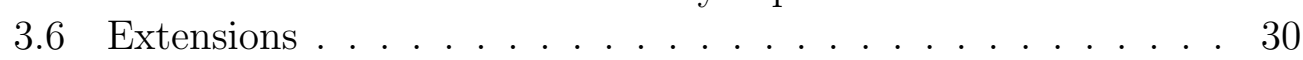

3.6.1 The Theoretical Model of Chason et. al. . . . . . . . 30

3.6.2 Normal Incidence Quantum Dot Formation. . . . . . . . 30

3.7 Theorv of Simultaneously Rotated and Sputtered Surfaces . . 32

3.7.1 Pattern Suppression. Smoothening. and Roughening . . 33

3.7.2 Topography and Dvnamic Scaling . . . . . . . . . . 35 
4 Modelling for Computer Simulations 37

4.1 Sputtering Models . . . . . . . . . . . . . . . . . . . . . . . 38

4.1 .1 Cascade Model . . . . . . . . . . . . . . . . . . . . . 39

$4.1 .2 \quad$ HKGK Model ... . . . . . . . . . . . . . . . . . . . . . . . . 39

4.1 .3 Combined Models . . . . . . . . . . . . . . . . . . . . . 40

4.2 Surface Diffusion Models . . . . . . . . . . . . . . . . . . . . 42

4.2 .1 Continuum Description . . . . . . . . . . . . . . . . 43

4.2 .2 Limited Mobilitv Models . . . . . . . . . . . . . . . . . 44

4.2 .3 The Hamiltonian Model . . . . . . . . . . . . . . . . . 47

4.2 .4 The Arrhenius Model . . . . . . . . . . . . . . . . . . . 49

5 Monte Carlo Simulations with the HKGK Model 52

5.1 Surface Erosion . . . . . . . . . . . . . . . . 53

5.1 .1 Model extension: lateral erosion _ . . . . . . . . . . 55

5.1.2 Model extension: sample rotation . . . . . . . . . . . 56

5.2 Hopping dvnamics . . . . . . . . . . . . . . . . . . . . 57

5.2 .1 Hamiltonian Model . . . . . . . . . . . . . . . . . 58

5.2 .2 Arrhenius Model . . . . . . . . . . . . . . . . . . . 59

6 Results and Discussion 63

6.1 Ripple Coarsening and Propagation . . . . . . . . . . . 64

6.1 .1 Ripple Kinematics . . . . . . . . . . . . . . . . 64

6.1 .2 Results . . . . . . . . . . . . . . . . . . . . 66

6.2 Morphological Regions . . . . . . . . . . . . . . . . . . . 71

6.2 .1 Possible Topographies . . . . . . . . . . . . . . . . . 73

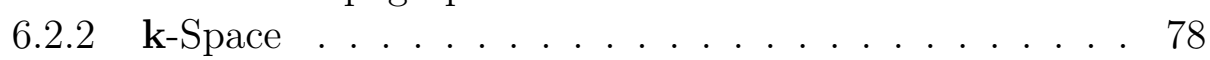

6.2 .3 Roughness and growth exponent . . . . . . . . . . 8 85

6.3 Rotated Substrate. . . . . . . . . . . . . . . . . . 86

6.3 .1 Profiles and discussion . . . . . . . . . . . . 87

7 Conclusions 94

7.1 Ripple coarsening and motion . . . . . . . . . . . . . . 95

7.2 Morphological regions and oblique incidence dot formation . . 96

7.3 Rotated substrates . . . . . . . . . . . . . . . . . . . 9 97

7.4 Outlook . . . . . . . . . . . . . . . . . . . 98

$\begin{array}{ll}\text { Bibliography } & 100\end{array}$

$\begin{array}{ll}\text { Curriculum Vitae } & 107\end{array}$ 


\section{List of Figures}

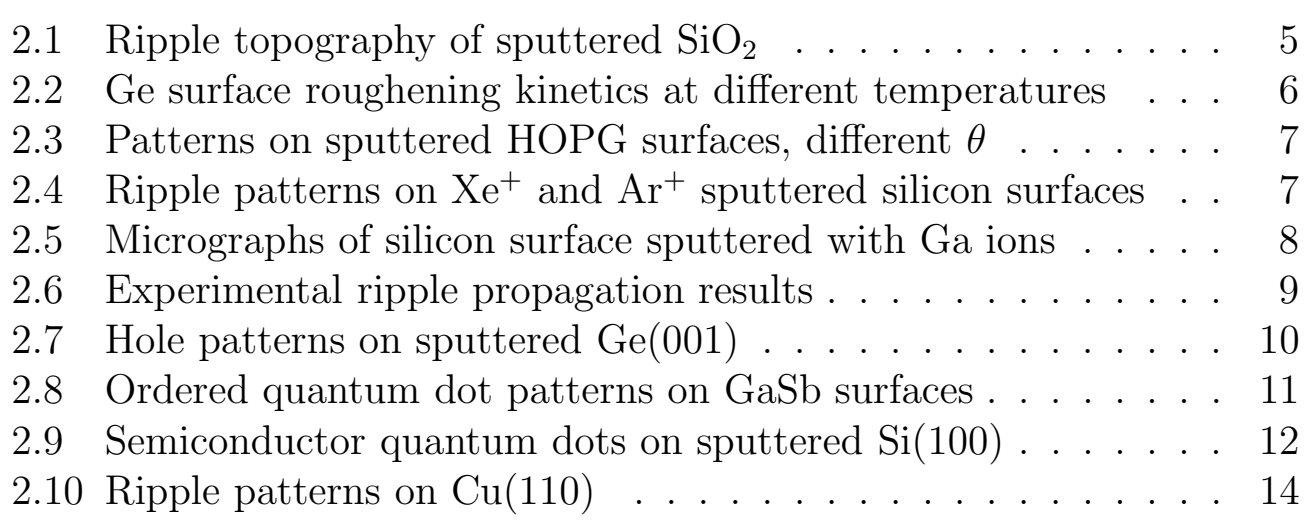

3.1 Illustration of scaling regimes . . . . . . . . . . . . . . 18

3.2 Plots of $h(x, t+\delta t)$ versus $x \ldots \ldots \ldots \ldots 20$

3.3 Ripple orientation phase diagram in the linear regime . . . . . 25

3.4 Ripple orientation phase diagram in the nonlinear regime . . . 28

3.5 Topography evolution from cellular to dot/hole structures . . 32

4.1 Discretization of a conserved growth equation . . . . . . . . . 46

4.2 The Hamiltonian model in $1 D \ldots \ldots$. . . . . . . . . . . . . . . . . . . 48

4.3 The Arrhenius model in $1 D$. . . . . . . . . . . . . 50

5.1 Reference frames and energy ellinsoid . . . . . . . . . . . . . 53

5.2 Dependence of ripple orientation on $\theta$ and $\phi$. . . . . . . 54

5.3 Inclusion of lateral erosion . . . . . . . . . . . . . . . . 55

5.4 Effect of stronger non-linearities on the surface width . . . . . 56

5.5 Effect of sample rotation . . . . . . . . . . . . . . 57

5.6 Profiles using Hamiltonian model. $T=0.2 \mathrm{Jk}_{D}^{-1}$. . . . . . . . . 58

5.7 Surface profile after long time sputtering $(\mathrm{t}=100) \ldots 60$

5.8 Profiles using Arrhenius dynamics, $T=0.1 \mathrm{eV} / k_{D} \ldots$. . . . . 61

6.1 Clustering of ripple crest points . . . . . . . . . . . 65

$6.2 \lambda(t)$ and $v(t)$. with Arrhenius dvnamics, $k_{R} T=0.1 \mathrm{eV}$. . . . 66

6.3 Higher-order linear coefficients in the continuum theory . . . . 67

$6.4 \lambda(t)$ and $v(t)$. with the Hamiltonian model. $\left.k_{R} T=0.2 \mathrm{~J}\right] . .68$

$6.5 \lambda(t)$ and $v(t)$. with Arrhenius dvnamics, $k_{R} T=0.2 \mathrm{eV}$. . . . . 69

6.6 Ripple velocity versus wavelength . . . . . . . . . . 69

6.7 Ripple velocity as a function of time, at different temperatures 70 
$6.8 \quad \lambda$ as a function of time. different T . . . . . . . . . . . 72

6.9 Sketch of the topographic phase diagram ... . . . . . . . 73

6.10 Profiles for each topographic region in Fig. 6.9 . . . . . . . 74

6.11 Surface profiles of region I (rough surface) . . . . . . . . 75

6.12 Surface profiles of the hole region (II) . . . . . . . . . . . 76

6.13 Profiles of the ripple region (III) at verv early times . . . . . 77

6.14 Profiles for a smaller $\theta$. . . . . . . . . . . . . 77

$6.152 \mathrm{D} \mathrm{S}(\mathrm{k})$ of phase space (Fig. 6.9 ) along $\mu=4$. and $\sigma=3 \ldots 79$

6.16 Separation of the $2 \mathrm{D} \mathrm{S}(\mathrm{k})$ into regions. for analvsid . . . . . 80

6.17 Plot of the quantities $a_{\mathrm{r} / \mathrm{h}}$ and $Q$ as functions of $\sigma \ldots \ldots$. . . . 81

6.18 Plot of the quantities $q_{\perp} \|$ and $Q$ as a function of $u \ldots \ldots 82$

6.19 Plot of the quantities $\alpha_{\nu_{h}}$ and $\theta_{\nu_{r}}$ as functions of $\sigma \quad \ldots .83$

6.20 Plot of $\nu_{x}$ and $\nu_{y}$ of the KS equation . . . . . . . . . 84

6.21 Time evolution of the surface width for the ripple region . . . 85

6.22 Time evolution of the surface width for the dot region . . . . . 86

6.23 Concurrent sputtering and rotation (PSSR) $\mathrm{t}=3 \ldots \ldots$. . . 88

$6.242 \mathrm{D}$ structure factor of the PSSR at $t=3 \quad \ldots \ldots . . .289$

6.25 Coefficients of the iKS equation for the rotated case . . . . . 90

6.26 Time evolution of rough surface with sample rotation . . . . . 91

6.27 Time evolution of holes with sample rotation . . . . . . . . . 92

6.28 Time evolution a non-oriented structure with sample rotation 92

6.29 Time evolution the dot structures with sample rotation . . . . 93 
Height, width, and depth

are the three phenomena

which I must transfer into

one plane to form the $a b$ -

stract surface of the pic-

ture, and thus to protect

myself from the infinity of space.

\section{Max Beckmann}

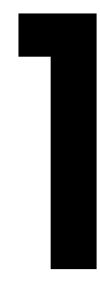

Introduction

Surface morphology evolution is a major focus of extensive research on nonequilibrium processes at surfaces. One such non-equilibrium process is surface growth, whereby a solid surface advances through the addition of new material, that crystallizes in a manner dictated by the lattice structure of the underlying solid [1. Another non-equilibrium process is surface sputtering, a process by which material is removed from a solid surface through the impact of energetic particles. Both processes drive the material surface out of equilibrium, and, apart from the academic interest in a better understanding of nonequilibrium systems, are widely studied partly due to the importance of interface structure to the synthesis of multilayer thin-film semiconductor devices, for opto-electronics and other applications.

The interest of the material physics community on surface sputtering, in particular, is partly due to its critical role in the fabrication [2] and analysis [3] of surface structures of technological importance at nanometer length scales. And partly due to the self-organization and ordering of the patterns that emerge from the stochastic ejection of material from the surface; i.e, correlation from randomness [4]. Thus, understanding the effects of particle radiation on solid surfaces is one of the fundamental problems in materials science [5, 6], and would enhance the control of the technologically important process of nanopatterning. Computer simulations are of vital importance in the study of natural phenomena, as they bridge the gap between experiments and analytic theories. This study concerns the modelling and simulation of surface features arising from ion beam bombardment, as well as the morphology of the surface itself, with prolonged sputtering. Thus, several discrete atomistic models are examined, in line with experimental results, and the continuum theory. The most successful atomistic model, for simulating ioninduced sputtering of surfaces at nanometer length scales (in particular at high fluences), is the one introduced by Hartmann, Kree, Geyer, and Kölbel, 
which we shall simply refer to as the HKGK model. This model is based on the same assumptions as the continuum models, and is applied in this work to study problems of interest. Note that the continuum theory is, so far, the most successful analytic theory that provides explanations for the behavior of sputtered, out-of-equilibrium, surfaces. Thus, it is the only analytic theory we shall consider in this study. Another possibility is to use linear response theory, but this has been ruled out as a viable quantitative theory of radiation erosion [4].

This work is organized as follows: In the next chapter, we discuss a few experiments from which we can summarize the overall, common features of sputtered surfaces. In our discussion, we consider amorphous and semiconductor (single, and AIII/BV alloys) materials, and metallic materials, as separate groups, in order not to confuse the reader with differing characteristics which at times arise due to the different classes of materials. A summary of the main features is provided at the end of the chapter, so as to indicate our focus in the discussion of the continuum theory.

In chapter 3, we shall discuss the main ideas of the linear and non-linear continuum theory, starting with the dynamic scaling of interface fluctuations. While the linear continuum theory is very successful in describing the time evolution of sputtered surfaces, and quantitatively explains the results of some experiments, its quantitative predictions do not agree with others. Thus, we shall discuss a few extensions, of the continuum theory, that has been proposed to account for the formation of semiconductor quantum dots. Finally, we shall discuss the theory of simultaneously rotated and sputtered surfaces.

Having examined the experimental results and the continuum theoretical explanations, we proceed in chapter 4, to a consideration of discrete atomistic models that may be employed to simulate the naturally discrete surface at nanometer length scales. We shall consider sputter erosion models, and surface diffusion models. Also, we shall consider those models that combine both sputtering and surface diffusion in a single iterative step. In chapter 5. we shall discuss how we have implemented the sputter-erosion model and surface diffusion models used in our work. In this chapter we focus mainly on the HKGK sputter-erosion model and the two more realistic surface diffusion models. Extensions of the HKGK model to include lateral erosion and sample rotation are also considered.

In chapter [6, the results of our simulations are presented and discussed. In the chapter, we first studied ripple motion after which we used the extended versions of the HKGK model to explore all possible topographies, constrained by experimental parameters that lead to pattern formation; as well as the effects of sample rotation on these possible topographies. Our main results 
are as follows. In our study of ripple propagation, we found a transition from the already known power-law behavior of the ripple wavelength as a function of time, at low temperatures, to a more rapid exponential behavior, at higher temperatures. The higher temperature behavior which we reported for the first time, to our knowledge, has recently received an experimental verification. However, the precise temperature at which the transition occurs remain an open question, which we believe can be answered through simulations with our model, in further studies.

By varying the collision cascade parameters in our model, we observe six different possible topographies, corresponding to six parametric regions. The variation is done within the framework of simulations of the stopping and range of ions in matter (SRIM); which reveal the values, of these collision cascade parameters, that are realistic as pertains to experiments. We found an upper bound, in the lateral straggle of the ion beam after penetration, for clear ripple formation. Beyond this upper bound, we found the formation of nano-sized islands, that are expected to exhibit quantum confinement; i.e, for off-normal incidence sputtering without sample rotation. But we found these dots to be formed with some background noise, in the form of one-dimensional structures oriented parallel to the ion beam direction. Subsequent inclusion of sample rotation then shows that rotation only destroys any anisotropy in the system.

Finally, in the last chapter, we give a general conclusion with a few suggestions for further studies. 
All life is an experiment.

The more experiments you

make the better.

Ralph Waldo Emerson

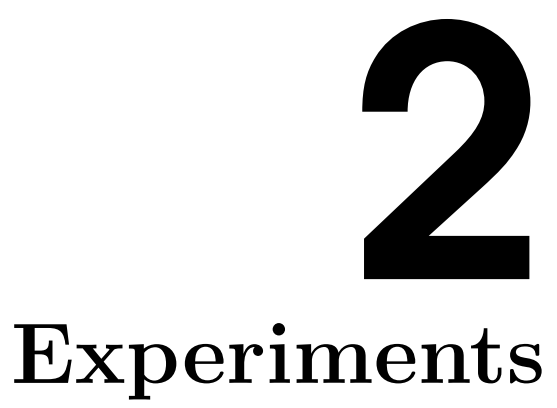

There is a huge amount of literature on the experiments studying the effects of material surface morphology induced by ion bombardment, at nanometer length scales, ion fluxes of the order of $10^{15}$ ions $\mathrm{cm}^{-2} \mathrm{sec}^{-1}$, and time scales of minutes. In this chapter, we present an overview of the common features of the topography evolution on amorphous, single semiconductor, alloy semiconductor, and metallic materials; i.e those features that are relevant to this work.

\subsection{Amorphous and Semiconductor Materials}

The bombardment of solid surfaces by a beam of energetic particles can lead to the production of atomic recoils and defects within the solid and the ejection or sputtering of surface atoms, with the consequent erosion of the solid. But, in addition, surface atomic relaxation effects which may be mediated by the irradiation, and/or mediated by surface diffusion also occur, hence, the morphology of the surface may be very complex [7].

Evidence of ripple structures on the surfaces of sputtered amorphous $\mathrm{SiO}_{2}$ and crystalline single semiconductor Ge surfaces have been provided in experiments by Chason et. al. [8, 9, 10, 11. In these experiments ion incidence angles $\theta=55^{\circ}-60^{\circ}$, and fluxes $\sim 10^{13} \mathrm{~cm}^{-2} \mathrm{sec}^{-1}$ were used. They observed a sputter yield $\approx 5 \mathrm{SiO}_{2}$ molecules/ion, which was considerably reduced to 0.1 molecules/ion with light ion $(\mathrm{H})$ bombardment. The three-dimensional structure of the roughened surfaces were examined ex-situ with atomic force microscopy (AFM), where a well defined ripple topography was found (Fig. 2.1) with the wave vector of the ripples being parallel to the direction of the incident ion beam [8]. They used in situ x-ray reflectivity to investigate $\mathrm{nm}$ 
scale variations in the morphology of $\mathrm{SiO}_{2}$ surfaces as a result of $\mathrm{Xe}$ and $\mathrm{H}$ ion bombardment, at $500^{\circ} \mathrm{C}$ [9]. During the bombardment of nominally smooth surfaces by $1 \mathrm{keV}$ Xe, they found a linear increase of the surface roughness with the fluence (the fluence is the number of incoming ions per unit area). On the other hand, bombardment of initially rough surfaces by $0.2-1 \mathrm{keV}$ $\mathrm{H}$ results in an exponential decrease in roughness with fluence, at a rate that increases with the energy. This smoothening by $\mathrm{H}$ ion bombardment has a rate that is also energy dependent, but the energy dependence is different for both cases, which rules out a simple relation between material removal and surface morphology. The observed ripple structure, however, is not resolved on a higher length scale [see Fig. 2.1 (b)].
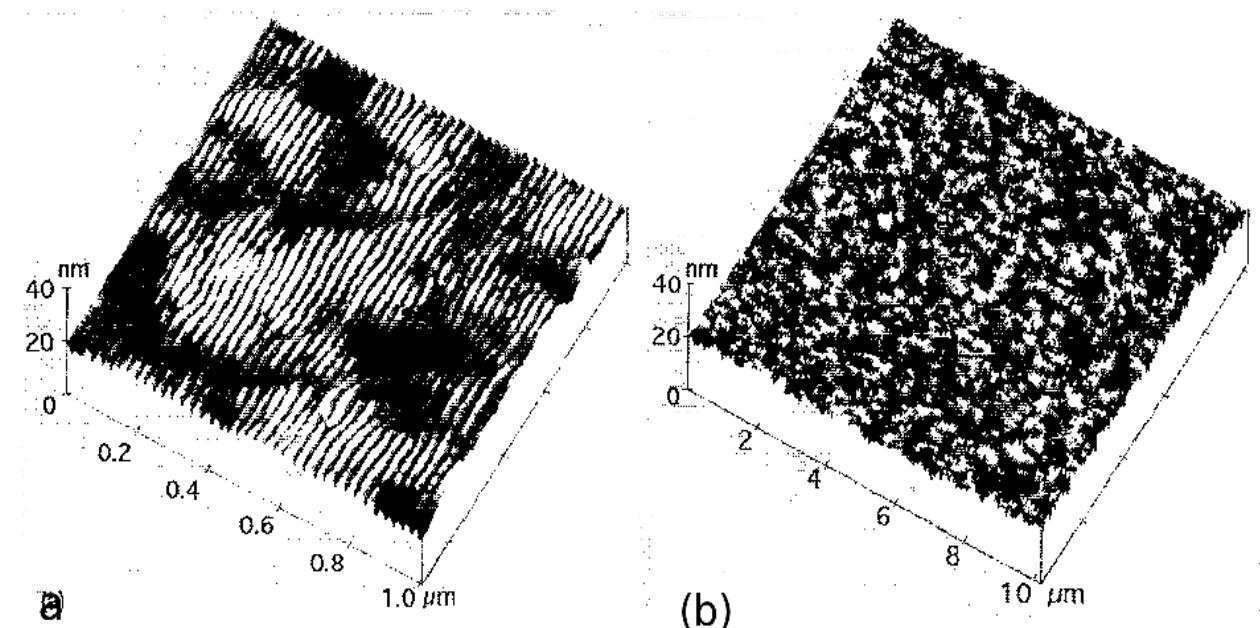

Figure 2.1: (a) $1 \mu \mathrm{m}^{2}$ AFM image of a Xe sputtered steam grown $\mathrm{SiO}_{2}$ film. The ion beam is incident at an angle of $54.9^{\circ}$ from normal (at room temperature), with surface projection perpendicular to the observed ripples. (b) $100 \mu \mathrm{m}^{2}$ AFM image of same sample as in (a); the ripple structure of (a) is not resolved on this length scale, but a random texture is quite evident with rms roughness comparable to (a), with no preferred orientation or frequency. (After Mayer et. al. [8]).

They also observed ripples, oriented perpendicular to the ion beam direction and with wavelengths on the order of $200 \mathrm{~nm}$, on sputtered crystalline Ge surfaces [11. At a higher temperature of $350^{\circ} \mathrm{C}$, the surface roughens exponentially with time, with an initial sublinear time dependence. The surface remains crystalline during sputtering at $250^{\circ} \mathrm{C}$ and above; the roughening does not saturate in this crystalline phase whereas it does in the amorphous phase, clearly demonstrating differing roughening and smoothing kinetics 
depending on the structure of the surface. At $250^{\circ} \mathrm{C}$ (see Fig. 2.2) the exponential roughening is faster. At $150^{\circ} \mathrm{C}$ and below (see Fig. 2.2), they observed that the Ge surface is amorphized by the ion impact and the roughness saturates (the flux remaining fixed at $2.5 \times 10^{12} \mathrm{~cm}^{-2} \mathrm{sec}^{-1}$ for this, and higher temperatures) to a steady state small value that does not increase even after prolonged sputtering.

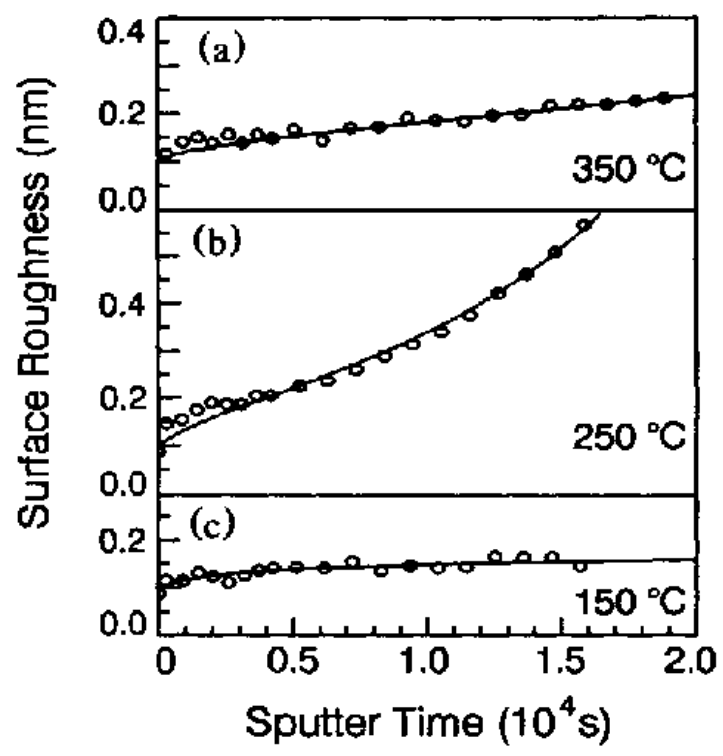

Figure 2.2: Ge surface roughening kinetics at different substrate temperatures. For $1 \mathrm{keV}$ Xe ion sputtering with flux $3.2 \times 10^{12} \mathrm{~cm}^{-2} \mathrm{sec}^{-1}$. Solid lines are the results of theoretical calculations discussed in their paper. The surface is crystalline, and the roughening is exponential in (a) and (b). The surface is amorphized in (c), and the roughening saturates. (After Chason et. al. [11])

Habenicht et. al., in their experiments on Xe-ion sputtering of graphite surfaces, found ripples oriented perpendicular to the ion beam for $\theta<\theta_{c}$ [Figs. 2.3 (a) and (b)]. For $\theta>\theta_{c}$, they found ripples oriented parallel to the ion beam direction [Fig. 2.3 (c)]. They found that $\theta_{c}$ lies between $60^{\circ}$ and $70^{\circ}$. The wavelength of these ripples were between $40-70 \mathrm{~nm}$, and the ripples were found to vanish with increasing ion fluences. At such high fluences, where the ripples vanish, they found that the surface exhibits a roughness governed by the Kardar-Parisi-Zhang (KPZ) equation. Ripple structures whose orientation depends on the ion beam direction, as described above, have been found on numerous other sputtered surfaces of amorphous 

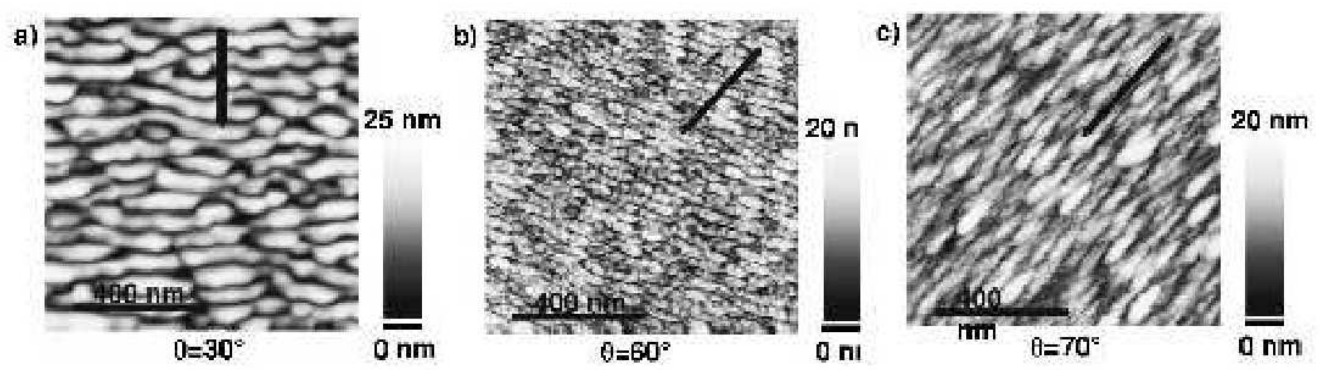

Figure 2.3: $\quad$ STM micrographs (lateral size $1 \mu \mathrm{m}$ ) of $5 \mathrm{keV} \mathrm{Xe}^{+}$eroded HOPG surfaces. (a) - (c): irradiation fluence of $3 \times 10^{17}$ ions $\mathrm{cm}^{-2}$; incident angle $\theta=$ $30^{\circ}, 60^{\circ}$, and $70^{\circ}$, respectively. (After Habenicht et. al. [12]).

materials, single semiconductor materials [13, 14, 15, 16, 17, 18, 19, and AIII/BV-alloy semiconductor materials [20]. In Ref. 20], the ripples were found to vanish with increasing temperature; and for InP, extensive cone formation (no ripples) occurs at $100^{\circ} \mathrm{C}$. Also, no ripple formation occurs when the sample is cooled to $-30^{\circ} \mathrm{C}$, where surface diffusion is limited. Thus, Ref. [20] shows the importance of surface diffusion in sputter-induced ripple and cone development.
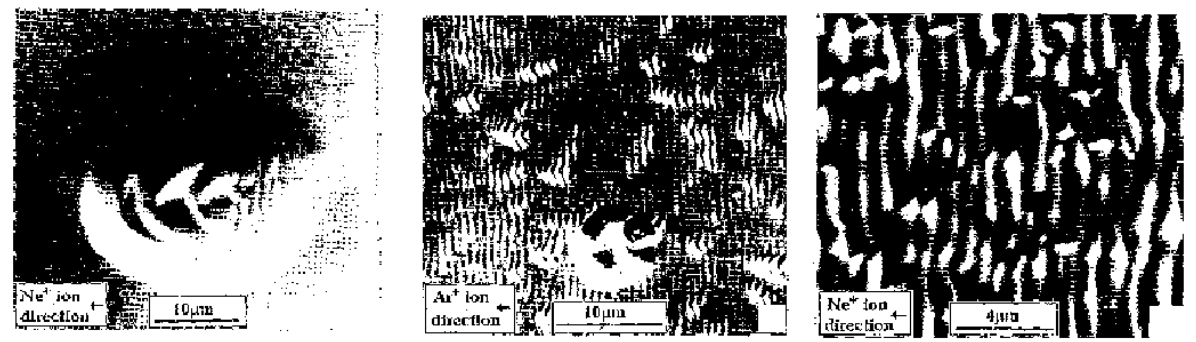

Figure 2.4: Topography of sputtered silicon surfaces. Left: implantation with 20 $\mathrm{keV} \mathrm{Ne}$ at room temperature, fluence $4 \times 10^{20}$ ions $\mathrm{cm}^{-2}$. Middle: implantation with $20 \mathrm{keV} \mathrm{Ar}{ }^{+}$at room temperature, fluence $3 \times 10^{19}$ ions $\mathrm{cm}^{-2}$. Right: implantation with $20 \mathrm{keV} \mathrm{Ne}$ at $120 \mathrm{~K}$, fluence $1.7 \times 10^{19}$ ions $\mathrm{cm}^{-2}$. In all cases $\theta=45^{\circ}$. (After Carter et. al. [21]).

In contrast to the low-energy studies, high energy bombardment of $\mathrm{Si}$ surfaces by Carter et. al. at room temperature produced ripples only with $\mathrm{Ar}$ ions 21. With the other inert gas ions (Xe, $\mathrm{Ne}$ ) ripples were found for temperatures below room temperature $(120 \mathrm{~K}$ or $200 \mathrm{~K})$, using the same ion 
characteristics (see Fig. 2.4). Their observation was done with scanning electron microscopes (SEM) and AFM. They observed that the ripples propagate on the surface with a vanishingly small velocity, which was not measured [14]. At $0^{\circ}$ incidence and to much higher fluences, surface roughness was much less and there was no sign of any periodic ripple structures. They found no ripple formation for incidence angle 0 - 40 . AFM observations of irradiated surfaces for all bombardment temperatures showed no changes in the surface morphology as a function of time after cessation of bombardment (in agreement with Ref. [8] ).

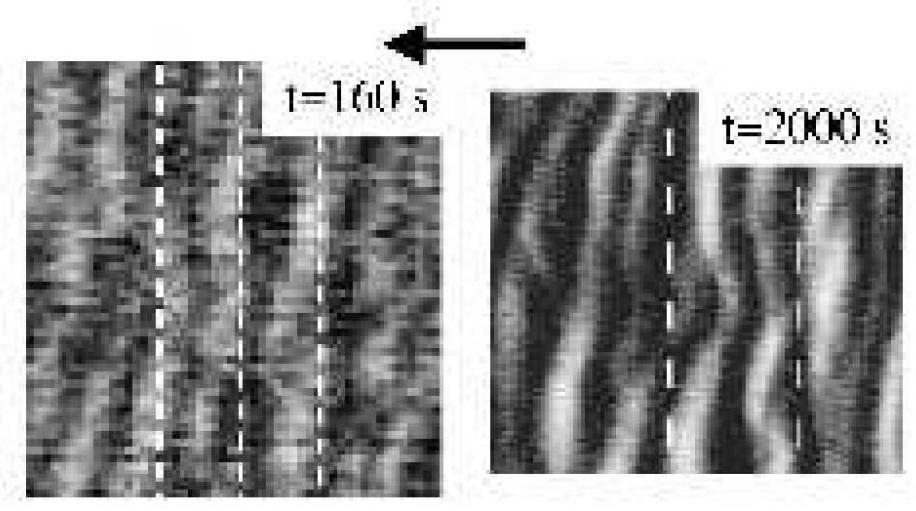

Figure 2.5: Focussed ion beam micrographs of silicon surface sputtered with 30 $\mathrm{keV} \mathrm{Ga}$ ions. The arrow indicates the projection of the ion beam onto the surface. The erosion times are indicated on the profiles. (After Habenicht et. al. [22]).

Ripple propagation was quantitatively measured by Habenicht et. al. in Ref. 22], where they also observed and measured the coarsening (i.e increasing wavelength) of the ripples with time. Later on, we compare our results with the results of this experiment, hence, we will discuss it in more detail than the other experiments. They monitored the evolution of $30 \mathrm{keV}$ Ga-ion sputtered $\operatorname{Si}(100)$ surfaces, $\theta=30^{\circ}$, in real time using $\mathrm{SEM}$ and focussed ion beam microscopy (FIBM) [22]. The typical ion current density was $7.5 \times$ $10^{14} \mathrm{~cm}^{-2} \mathrm{sec}^{-1}$; the current density has been varied between $10^{14}$ and $10^{15}$ $\mathrm{cm}^{-2} \mathrm{sec}^{-1}$. In this experiment, the ripples were observed to propagate, on the surface plane, parallel to the ion beam direction (Fig. 2.5). Contrary to the linear continuum theory, they found that the ripple wavelength $\lambda$ is not constant with time. For erosion time $t \approx 1-135 \mathrm{~min}$, a ripple coarsening (Fig. 2.5) of the form $\lambda \sim \mathrm{t}^{0.5}$ was found [see Fig. 2.6] (a)]. They also performed a quantitative study of the ripple motion and found a nonuniform ripple velocity $v$. After an initial plateau of constant velocity, for $\lambda \gtrsim 100$ 

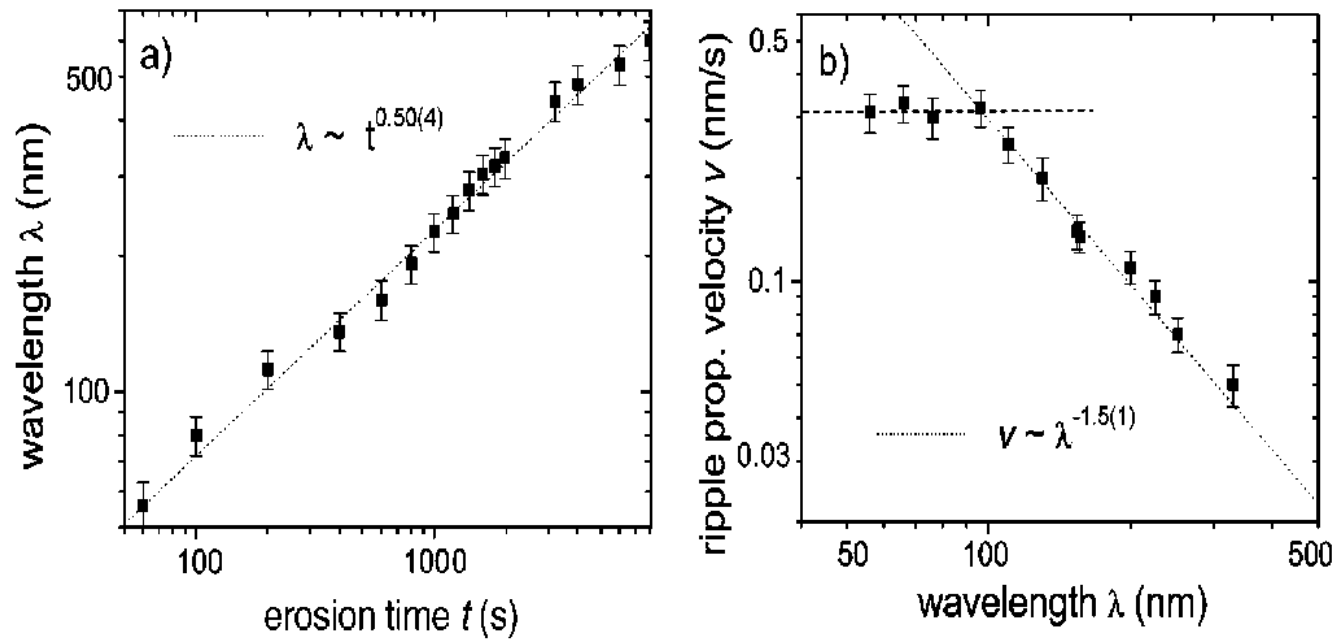

Figure 2.6: Ripple coarsening, and velocity dispersion. (a): measured ripple wavelength as a function of t. (b): measured velocity dispersion of the ripple propagation with rising wavelength. (After Habenicht et. al. [22]).

nm, a power-law behavior $v \sim \lambda^{-1.5}$ was found [seeFig. 2.6 (b)].

However, instead of ripples, a relatively disordered arrangement of mounds were found by Chey et. al. [23, 24]. They studied crystalline germanium surfaces, during low-energy sputtering, using in situ scanning tunneling microscopy (STM) 23. The Ge(001) surfaces were bombarded by $240 \mathrm{eV} \mathrm{Xe}$ ions, with a flux 1 of $1.6 \mu \mathrm{A} \mathrm{cm} \mathrm{cm}^{-2}$, and inclination $50^{\circ}$ to the vertical. They obtained a sputter yield of $0.7 \mathrm{Ge}$ atoms per Xe-ion impact. At $\mathrm{T} \approx 270^{\circ} \mathrm{C}$ the character of the surface morphology changes from the relatively disordered arrangement of mounds to a more regular pattern of pits (see Fig. 2.7).

On the other hand, no surface pattern (neither ripple nor pits) was reported in the experiments of Eklund et. al. 4], on the off-normal incidence $\left(\theta=60^{\circ}\right)$ sputtering of graphite surfaces with $5 \mathrm{keV}$ Ar ions. The experimental parameters varied in the study were the ion flux $\mathrm{J}\left(6.9 \times 10^{13}\right.$ ions $\mathrm{cm}^{-2} \mathrm{sec}^{-1}$, and $3.5 \times 10^{14}$ ions $\left.\mathrm{cm}^{-2} \mathrm{sec}^{-1}\right)$, the fluence $Q=J t\left(10^{16}\right.$, $10^{17}$ and $10^{18}$ ions $\left.\mathrm{cm}^{-2}\right)$, and the substrate temperature $T(\approx 300,600$, and $900 \mathrm{~K})$. They used a scanning tunneling microscope (STM) to examine the sputtered surfaces. Instead of ripple structures, they found rough surfaces

\footnotetext{
${ }^{1}$ an ion/charge current density of $1 \mathrm{C} /\left(\mathrm{cm}^{2} \mathrm{sec}\right) \approx 6.24 \times 10^{18} \mathrm{ions} /\left(\mathrm{cm}^{2} \mathrm{sec}\right) \approx 1 \mathrm{~A} / \mathrm{cm}^{2}$, hence, $1 \mu \mathrm{A} / \mathrm{cm}^{2} \approx 6.24 \times 10^{12}$ ions $/\left(\mathrm{cm}^{2} \mathrm{sec}\right)$
} 

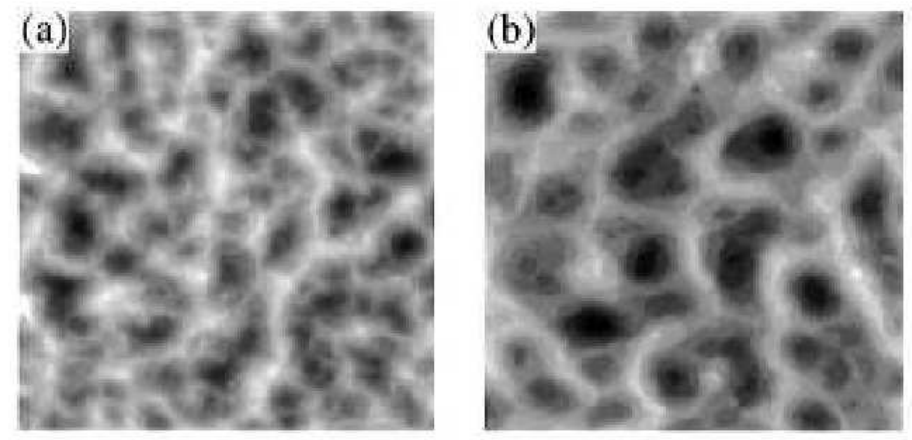

Figure 2.7: STM images of Ge(001) after $42 \mathrm{~min}$ sputtering with $240 \mathrm{eV}$ Xe ions at a substrate temperature of $270^{\circ} \mathrm{C}$. (b) Subsequent annealing at $270^{\circ} \mathrm{C}$ for 20 min. (After Chey et. al. 24]).

whose scaling is governed by the KPZ equation. They observed the slow (less than linear) divergence of the correlation length $\xi$ with the fluence; at the highest fluence $\xi$ exceeds the image size. Within the uncertainties of the experimental data, $\xi$ does not appear to depend on the flux.

Experiments by Facsko et. al. showed that normal incidence sputtering of the surfaces of semiconductor alloys, particularly gallium-antimonide (GaSb) and indium-antimonide (InSb) surfaces [25], can serve as a very attractive alternative to the production of self-organized semiconductor quantum dots in the Stranski-Krastanov growth mode in molecular-beam epitaxy and metalorganic vapor epitaxy; in which coherent island formation occurs during the growth of lattice-mismatched semiconductors. They found $\left(\theta=0^{\circ}\right)$ a highly regular pattern of hexagonal dots whose specific size and shape depended on the ion fluence (and energy, see Fig. 2.8). After about 40 secs. (equivalent to a fluence of $4 \times 10^{17} \mathrm{~cm}^{-2}$ ), small dots with an average diameter of 18 $\mathrm{nm}$ appear, as seen from the SEM images. Hexagonal ordering is already present in the shape, although some irregularities occur, and the dot density is $4.5 \times 10^{10} \mathrm{~cm}^{-2}$. The dot diameter grows to $34 \mathrm{~nm}$ up to an exposure time of 200 secs. (fluence of $2 \times 10^{18} \mathrm{~cm}^{-2}$ ). After 400 secs. the dot diameter was $50 \mathrm{~nm}$, approximately equaled the period, the hexagonal order is fully developed and represents the final stage of the dot formation.

The pattern does not change further, for longer exposure times, and the surface front propagates into the material with aconstant velocity of $1.5 \mathrm{~nm}$ $\mathrm{sec}^{-1}$ as the sample is eroded. A cross-sectional high-resolution transmission electron microscope (HTEM) image of the nanostructures (after 400 secs. exposure) revealed that the dots have a cone-like shape, and are covered by 


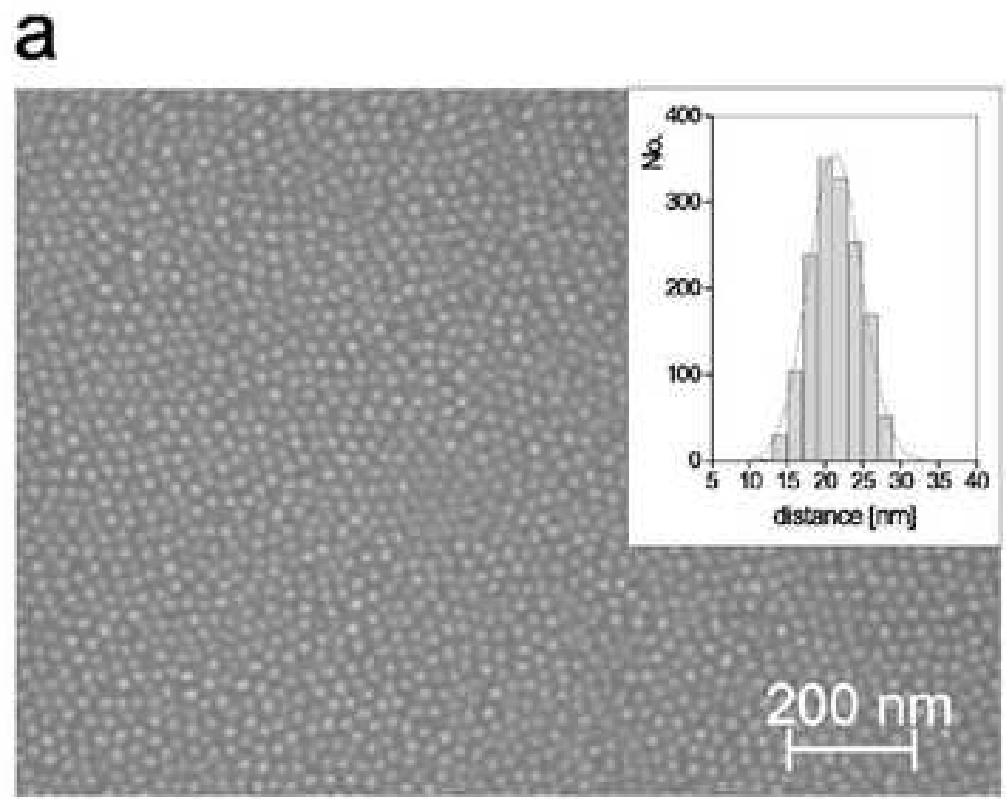

\section{b}

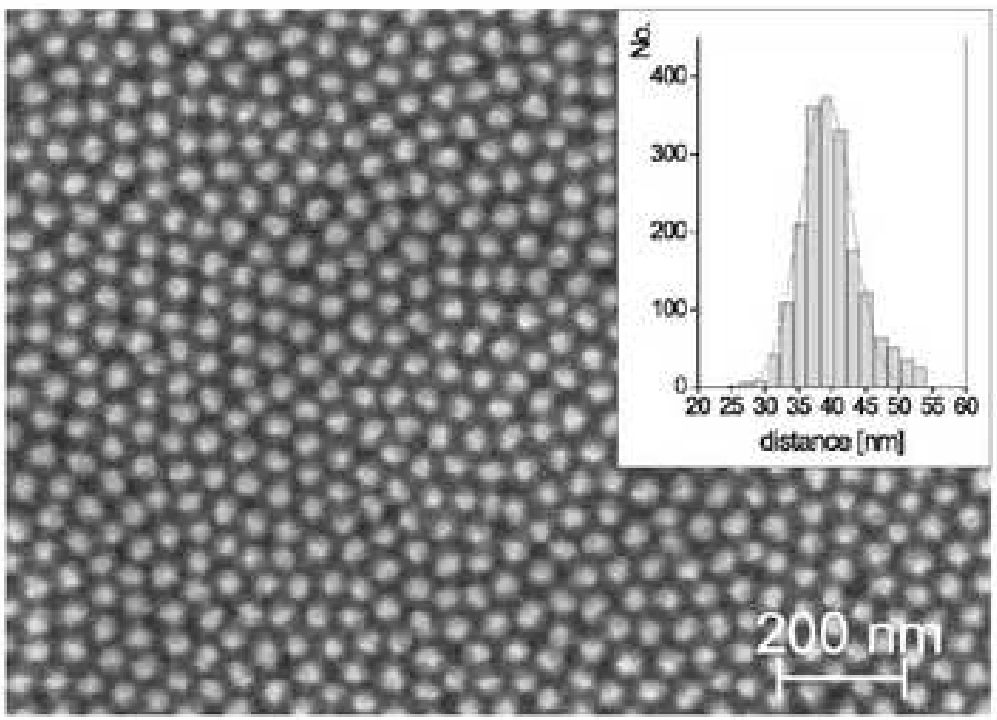

Figure 2.8: Scanning electron micrographs of quantum dot patterns on $\mathrm{GaSb}$ surfaces induced by $\mathrm{Ar}^{+}$sputtering with ion energies of (a) $100 \mathrm{eV}$, (b) $500 \mathrm{eV}$. The dots show an hexagonal ordering with a characteristic wavelength that depends on ion energy. The insets show the corresponding distribution of the nearest-neighbor distance [number of dots versus distance (nm)]. (After Facsko et. al. 27]). 
an amorphous layer of $\approx 2 \mathrm{~nm}$ thickness (a similar amorphous cover was found by Gago et. al., as shown in Fig. 2.91). The cone-like dots themselves
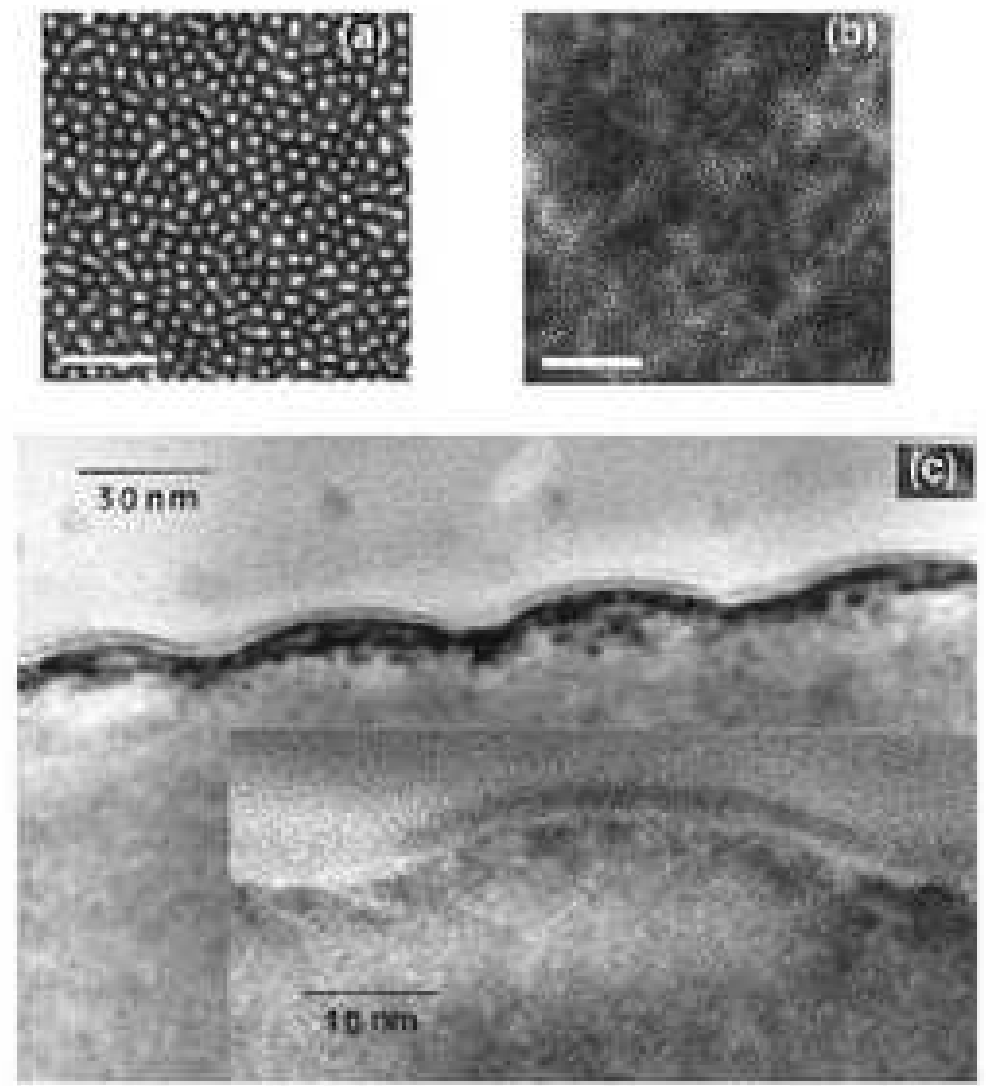

Figure 2.9: AFM images of $\mathrm{Si}(100)$ substrate sputtered by $1.2 \mathrm{keV} \mathrm{Ar}^{+}$at normal incidence for $6 \mathrm{~min}$ (a), and $960 \mathrm{~min}$ (b). The bars in (a) and (b) represent $277 \mathrm{~nm}$, and $831 \mathrm{~nm}$, respectively. (c): Cross-sectional HRTEM multibeam image along the $\langle 110\rangle$ direction of the sputtered sample; inset of (c): high-resolution image of one of the nanocrystals on the main panel. (After Gago et. al. [26]).

are crystalline, with the same orientation and crystalline structure as the GaSb substrate. For $\theta>0$, they observed ripples of wavelength $35 \mathrm{~nm}$. Similar results have been obtained by Gago et. al. [26] for normal incidence, low-energy sputtering of Si surface with Ar ions (see Fig. 2.9).

Formation of surface ripples is problematic in applications like secondary ion mass spectroscopy (SIMS), Auger electron spectroscopy (AES) and ion milling. SIMS is one of the most widely used techniques for the dopant 
profiling of semiconductors, AES is an important tool in the structural characterization of multilayers, and ion milling is a technique for shaping or removing unwanted parts on surfaces. In a typical SIMS or AES apparatus the primary ions are incident at $\theta>0$, which leads to ripple formation and, hence, to rapid degradation of the depth resolution, as well as changes in the secondary ion yield. Zalar [28] demonstrated that ripple formation can be overcome by simultaneous sputtering and sample rotation. Cirlin et. al. have shown that such sample rotation can remove ripple patterns, if they are already present on the surface before the onset of rotation, or suppress their formation, if rotation is started from the onset of sputtering [29]. In both cases the rotation can lead to a considerable smoothening of the surface. They also observed that sample rotation does not always suppress surface roughening, as the surface subjected to simultaneous sputtering and rotation still roughens in some cases, though at a slower rate than without rotation [3, 29]. With this insight from the experiments, we later implemented sample rotation in our simulations, to see its effect on the observed topographies.

With simultaneous sample rotation as the solid surface is sputtered, a highly regular hexagonal pattern of close-packed mounds or cones has been reported for oblique incidence sputtering of InP surfaces, by Frost et. al., using $500 \mathrm{eV}$ Ar ions incident at $\theta=40^{\circ}$ [30. The lateral size $\left(\lambda_{m}\right)$ and height of the cone-like structures increase with the fluence, or sputter time $t$, according to the power-law $\lambda_{m} \sim t^{0.26}$. But, for longer times, the mounds saturates (i.e. $\lambda_{m}$ becomes constant) and the topography changes from a more irregular to a highly regular hexagonal pattern of mounds. Without sample rotation, they did not find any evidence of ripple formation. Instead, they found cone-like oriented structures, where the orientation depends on the ion beam direction, as in the case of ripple formation.

\subsection{Metallic Materials}

Since this work is not concerned with metallic surfaces, the anomalous behavior of such surfaces (i.e relative to amorphous surfaces) is briefly discussed here, in order to have an overall picture of nm scale surface patterning with ion beams.

In contrast with the patterns observed on amorphous materials and semiconductors amorphized by the ion beam (described in the preceeding section), topographic features arising from ion sputtering of single metals, with isotropic surfaces, have been shown to reflect the substrate symmetry. For instance, under the same sputtering conditions as on amorphous surfaces (i.e low-energy ions, $\theta>0$ ) square pits have been observed on $\mathrm{Cu}(001)$ 31] and 
$\operatorname{Ag}(001)$ [32, 33]; hexagonal pits on $\mathrm{Pt}(111)$ [34], $\mathrm{Au}(111)$ [35], and $\mathrm{Cu}(111)$ 36. In the case of anisotropic metallic surfaces, the asymmetry induces a self-aggregation at the surface, leading to the formation of characteristic ripple-like structures whose orientation depends on a crystallographic direction. For instance, ripples with crests oriented along $\langle 1 \overline{1} 0\rangle$ have been found on $\operatorname{Ag}(110)$ 37.

The same dependence of the orientation on a crystallographic direction was observed for ripples patterned on a $\mathrm{Cu}(110)$ surface, provided $0<\theta<\theta_{c}$ (see Fig. 2.10). This was demonstrated by Rusponi et. al. in 32, 38, where
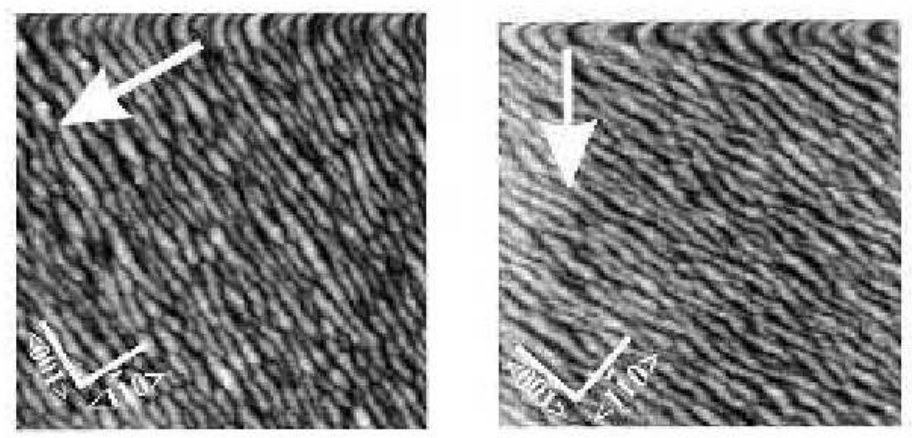

Figure 2.10: Ripple patterns on $\mathrm{Cu}(110)$ after ion sputtering at $\mathrm{T}_{S}=180 \mathrm{~K}$ for $\theta=45^{\circ}$. The arrow indicates the ion beam direction; the ripple orientation depends on a crystallographic direction. (After Rusponi et. al. [32]).

the wave-vector of ripples obtained from $1 \mathrm{keV} \mathrm{Ar}^{+}$sputtering of $\mathrm{Cu}(110)$ at $180 \mathrm{~K}$, were shown to be parallel to the $\langle 1 \overline{1} 0\rangle$ direction. But, for $\theta>\theta_{c}$, the ripple orientation depends only on the ion beam direction; independent of the crystallographic direction.

For $\theta=0$, at $T_{S}=180 \mathrm{~K}$, the surface is rough; at $250 \leq T_{S} \leq 270 \mathrm{~K}$, the surface is characterized by a well-defined ripple structure with $\lambda \approx 20$ $\mathrm{nm}$, and wave-vector parallel to $\langle 1 \overline{1} 0\rangle$; this ripple structure is degraded at $T_{S}=320 \mathrm{~K}$; and another ripple structure with $\lambda \approx 100 \mathrm{~nm}$, and wave-vector perpendicular to $\langle 1 \overline{1} 0\rangle$ is obtained for $350 \leq T_{S} \leq 360 \mathrm{~K}$.

\subsection{Summary}

In the experiments, different methods (STM, SEM, AFM, FIBM, RHEED, and x-ray reflectivity) have been used to probe sputtered surfaces at nanometerscales, and a range of ion fluences of the order of $10^{13}-10^{18}$ ions $/ \mathrm{cm}^{2}$. The 
X-ray based techniques allows the in situ and real time study of surface morphology. Whereas with the microscopes (e.g STM), the surface features have to be frozen by cooling to a lower temperature, usually around $200 \mathrm{~K}$, before the (e.g STM) topographs are taken. From studies of the time evolution of the morphology of various surfaces in these experiments one may draw the following general conclusions.

Stochastic surface particle removal or deposition roughens the surface and creates additional surface defects, whereas relaxation processes, via surface diffusion or radiation-induced viscous flow, smoothens the surface. The observed surface morphologies evolve as a result of a balance between these two processes. Normal incidence sputtering have been shown to be an attractive alternative, to lithographic techniques, in the production of uniform quantum dots on material surfaces. For off-normal incidence sputtering of amorphous/semiconductor surfaces, periodic ripples with a characteristic length scale have been observed. Such ripples are either oriented perpendicular to the ion beam projection on to the surface plane, for small incidence angles, or parallel to the projection, for incidence angles close to grazing. On other materials, also at off-normal incidence, an array of mounds and pits were found; no ripples. Other experiments have reported no surface patterns; no mounds, no ripples.

The patterns formed on metallic surfaces have been found to reflect the substrate symmetry; mound and pits on symmetric surfaces, and ripples (below room temperature) on asymmetric surfaces. For small incidence angles the ripples have been found to depend on the crystallographic direction, whereas for high incidence angles the ripples depend on the ion-beam direction. Formation of ripples on such asymmetric metallic surfaces, at $\theta=0$, depends on the substrate temperature; from rough surface at $T=180 \mathrm{~K}$, a ripple topography develops at $T=250 \mathrm{~K}$. This ripple is degraded at $320 \mathrm{~K}$, but another clear ripple of longer wavelength appears at $350 \mathrm{~K}$ with wave vector rotated by $90^{\circ}$.

As we shall see in the next chapter, some of these observations are well described by the continuum theory. In the next chapter, we shall discuss the major concepts of the continuum theory, and its successful description of surface morphology induced by ion bombardment. More detailed comparison of the theory to specific experiments can be found in Ref. 39. Also, as seen in some of the experiments, the time evolution of the surface roughness is of great interest, thus, in the next chapter, we shall first discuss the scaling of interface fluctuations before discussing the continuum theory. 
It is the theory that decides

what can be observed.

Albert Einstein

\section{3 \\ Continuum Theory}

\subsection{Introduction}

The continuum theoretical description of interface morphology pertains to modelling the evolution of the interface height by means of deterministic and stochastic partial differential equations. These equations are very powerful and successful tools for understanding the behaviour of diverse interface phenomena. They typically assume the interface to be defined by some continuous function (i.e, the interface is a continuum of points), which implies that one focusses only on the asymptotic coarse-grained properties, instead of the discrete short length-scale details; i.e, those "details" that do not affect the surface morphology. Continuum theory provides a good understanding of the interplay and competition between the processes that govern the interface evolution.

The continuum equations can be derived either from a discrete model of the system, if already known, or by exploiting symmetry principles [1]. The rest of the chapter is organized as follows: in the next section we start with a brief treatment of the scaling of interface height fluctuations, as is commonly referred to in the literature of various interface phenomena. Next, we discuss the linear continuum theory, followed by the non-linear continuum theory; as pertains to static sputtered surfaces. And, finally, we discuss the theory of rotated substrates. While these theories were proposed specifically for the self-organized nanopatterns on amorphous surfaces, a number of experiments on crystalline surfaces have demonstrated that the explanations and predictions apply to crystalline surfaces as well. 


\subsection{Scaling of Interface Fluctuations}

The roughness of many natural surfaces, or interface between two media, follow simple scaling laws, which are quantified using scaling exponents. The exponents characterize the time evolution of the roughness of an externally driven surface at small length scales; comprising of two different regimes: a growth regime (I), and a steady-state regime (II), of the surface roughness.

The surface is described by a discrete single-valued (i.e. no overhangs) function $h(\mathbf{r}, t)$ of the points $\mathbf{r}$ on the surface at time $t$. The surface roughness $W$ is defined to be the root-mean-squared (rms) fluctuation in the surface height $h(\mathbf{r}, t)$, i.e,

$$
W(L, t) \equiv \sqrt{\frac{1}{L^{d}} \sum_{\mathbf{x}}[h(\mathbf{x}, t)-\langle h(t)\rangle]^{2}},
$$

where $\langle h(t)\rangle$ is the average surface height at time $t$. The surface is expected to saturate, i.e, crossover to regime II, when the typical wavelength of these fluctuations $\xi(t)$ (also called the correlation length) is of the order of the system size; thus, the scaling hypothesis characterizes finite-size effects. Suppose we characterize regime I with the growth exponent $\beta$, then, $W=k_{1} t^{\beta}$ in regime I, and $W=k_{2}=W_{s}$ in regime II; where $k_{1}$ and $k_{2}$ are constants. Since $\xi(t)$ grows to $\xi(t) \sim L$, then we should expect the saturation time $t_{s}$ to grow with system size, which implies that $W_{s}$ must be size dependent. If we characterize this with the steady-state exponent, or (saturation) roughness exponent, $\alpha$, then $W_{s}=k_{3} L^{\alpha}$. To conclude, we can characterize the size dependence of $t_{s}$ with the time, or dynamic, exponent $z$; i.e, $t_{s}=k_{4} L^{z}$ (see Fig. 3.1). Thus,

$$
W(L, t) \sim \begin{cases}t^{\beta} & \text { for } t<<L^{z} ; \text { i.e, } t<t_{s} \\ L^{\alpha} & \text { for } t>>L^{z} ; \text { i.e, } t>t_{s}\end{cases}
$$

Indeed these power laws have been observed in numerous experiments on surface growth, e.g. 3D island formations, and 2D epitaxial growths during vapor deposition. They have also been observed in sputtering experiments. The three exponents $\alpha, \beta$ and $z$ are related, and this relation can be obtained by assuming that all scaling relations hold at the transition to steady-state roughness, i.e, combining $W \sim t_{s}^{\beta} \sim\left(L^{z}\right)^{\beta}$, with $W \sim L^{\alpha}$ gives the scaling law

$$
\alpha=\beta z .
$$

The relation (3.2) is often expressed with a scaling function $f(u)$, i.e,

$$
W(L, t)=L^{\alpha} f\left(\frac{t}{L^{z}}\right)
$$




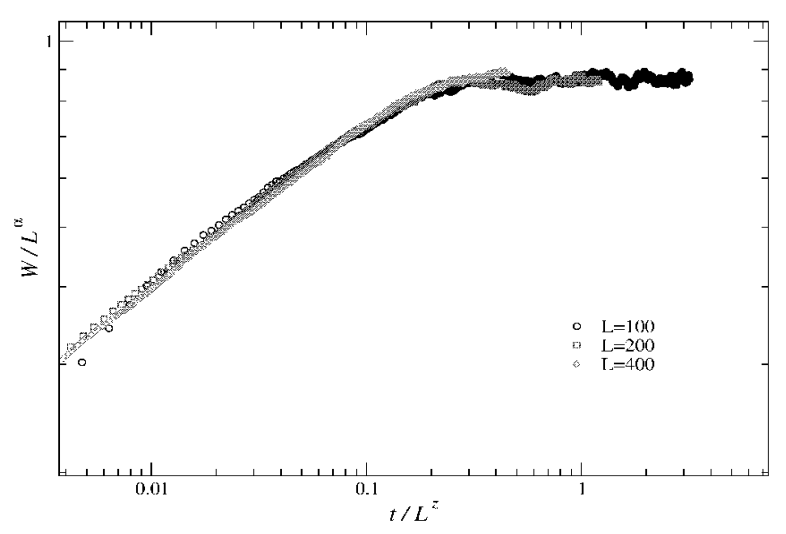

Figure 3.1: Illustration of the scaling regimes: data collapse for the ballistic deposition model in 1D. In the first regime, for the three different system sizes, the surface width $W$ increases with time $t$ as $W \sim t^{\beta}$, after which saturation is achieved at $t=t_{s} \sim L^{z}$. At $t_{s}$ a crossover to the steady state regime occurs, such that $W \sim L^{\alpha}$. To achieve a collapse of the data for the three system sizes, the width and time have been re-scaled according to: $W \rightarrow W / L^{\alpha}, t \rightarrow t / L^{z}$. We found $\alpha, \beta$, and $z$ to be $0.427,0.305$, and 1.4 , respectively.

where $f(u) \sim u^{\beta}$ for $u<<1$, and $f(u)=$ constant, for $u>>1$. The ballistic deposition growth model is a good example of the dynamic scaling, as saturation of the width is rapidly achieved. The result of simulations performed with this model is shown in Fig. 3.1 as an illustration of the two regimes.

Now, at saturation, $t=t_{s} \sim L^{z}$ due to $\xi \sim L$ (i.e, $L \sim \xi$ ). This implies $t_{s} \sim \xi^{z}$; or, $\xi \sim t_{s}^{1 / z}$. It should be noted that regime I can be splitted, for more complex systems, due to different rates, or non-linearities. For instance, the growth regime can consist of an initial transient (as in ballistic deposition, for $t<1$ [1]) in which poisson growth occurs with $\beta=1 / 2$. These exponents are believed to be universal, and unique to the specific process responsible for the surface morphology, independent of the microscopic details of the system; i.e, depending only on the conditions of height evolution (sputtering or growth).

To summarize, the morphology of the interface between two media, at least one of which is dense, exhibits dynamic scaling. The exponents of such scaling characterize, and are unique to, the specific process governing the morphology. Such a process (with exponents $\alpha_{p}$ and $z_{p}$, for instance), is universal (thus defining a universality class) in the sense that its presence elsewhere, in a seemingly completely different phenomenon, give rise to ex- 
actly the same scaling exponents $\left(\alpha_{p}\right.$ and $\left.z_{p}\right)$. At large lengthscales, however, the surface is smooth and independent of these exponents.

\subsection{Effect of Each Component of a General Continuum Equation}

In the continuum theory, the surface morphology is modelled with partial differential equations (PDE). We begin by discussing the effect of the coefficients of each terms in one such general, lowest order PDE. In a time interval, $\delta t$, the surface height changes by an amount $\delta h=h(\mathbf{r}, t)-h(\mathbf{r}, t+\delta t)$, given by

$$
\delta h=\nu \nabla^{2} h(\mathbf{r})-K \nabla^{4} h(\mathbf{r})+\frac{\lambda}{2}(\nabla h(\mathbf{r}))^{2},
$$

i.e in the absence of shot noise and anisotropies. The effects of each coefficient in Eq. (3.5) is explored in Fig. 3.2 for the 1D case. E.g, considering their effect on a single Fourier component of the height profile, $h(\mathbf{k}, t)=h_{0}(\mathbf{k}, t) \sin (\mathbf{k} \cdot \mathbf{r})$ where $\mathbf{k}=k \hat{x}$, and $\mathbf{r}=x \hat{x}$; the evolution of an arbitrary surface can then be understood as a superposition of the Fourier components. In the first term on the RHS, $\nabla^{2} h(\mathbf{r})$, being negative, relaxes the surface $h(\mathbf{r})$ by a factor of $\nu$; thus if $\nu<0$, the surface is unstable against any perturbation and the fluctuations (in the surface height) tend to grow [Fig. 3.2 (a)], thereby maximizing the exposed surface area, and reducing the ratio of the tangential force per unit area on the surface, which is why it is referred to as a negative surface tension. If $\nu$ is positive, the surface is stable against any perturbation, and relaxes as shown in Fig. 3.2 (a). In the second term, $\nabla^{4} h(\mathbf{r})$ increases $h(\mathbf{r})$ by an amount $K$. With the prefactor $-|K|$, it relaxes the surface as shown in Fig. 3.2 (b), for $K=0.1$ and 0.3. Without this [i.e $-K$ as in (3.5), the surface again grows, as for $K=-0.3$. This second term arises from a coarse grained continuity equation, and is discussed in section 4.2. The third term is the non-linear term $(\nabla h(\mathbf{r}))^{2}$, accounted for by the KPZ theory. It allows for some interesting localized control of the surface topography, as seen in the simple profile of Fig. 3.2 (c), since it can spread a surface protrusion, or flatten its peak, or reproduce two, albeit smaller but higher, copies of the protrusion, due to lateral growth, with increasing magnitude $\lambda$. However, if $\lambda<0$ [Fig. 3.2 (d)], the peak remains the same while the sides are eroded, so called lateral erosion. Note that $(\nabla h(\mathbf{r}))^{2}>0$, and tends to destabilize the surface; a trend that is reversed for $\lambda<0$.

Anisotropies give rise to different coefficients $\nu$, or $\lambda$, or $K$, along the different directions, and breaks the symmetry with respect to rotation, trans- 
(a)
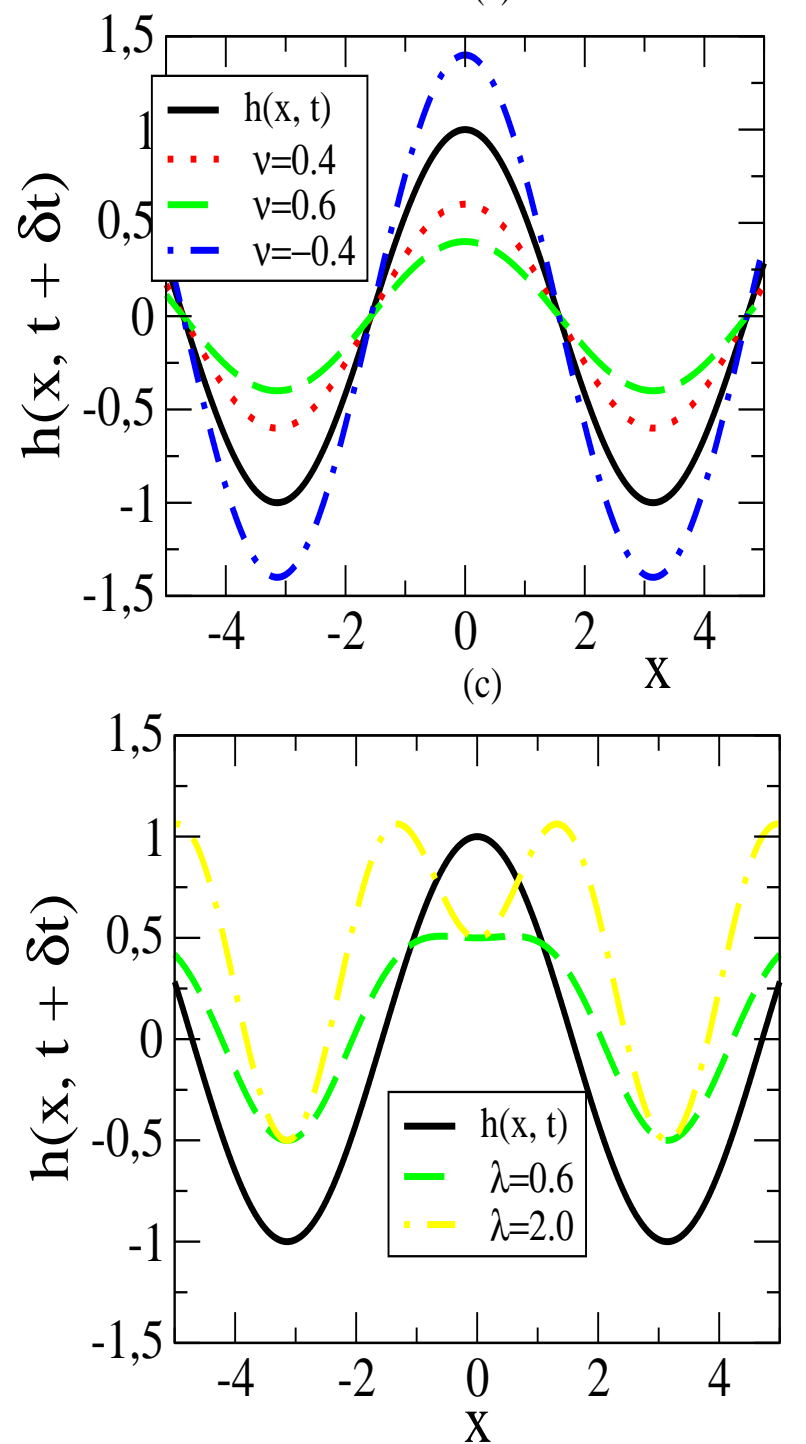

(b)

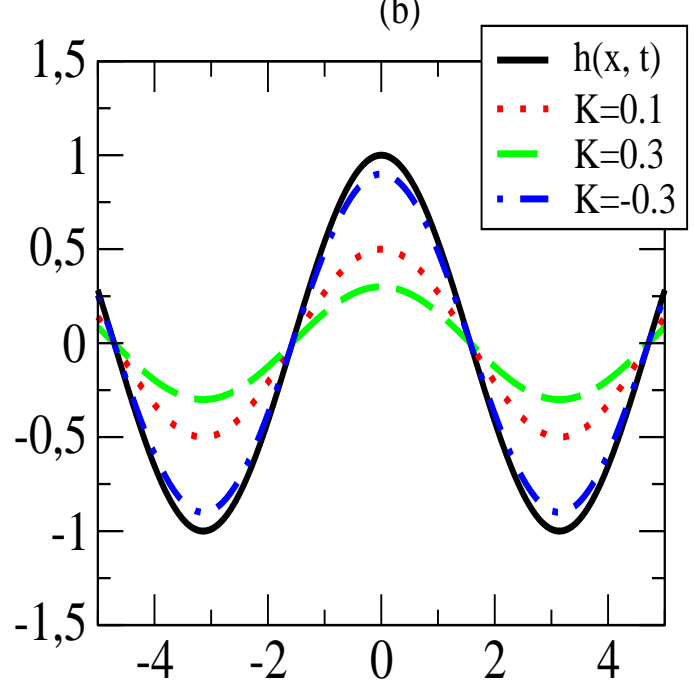

(d)

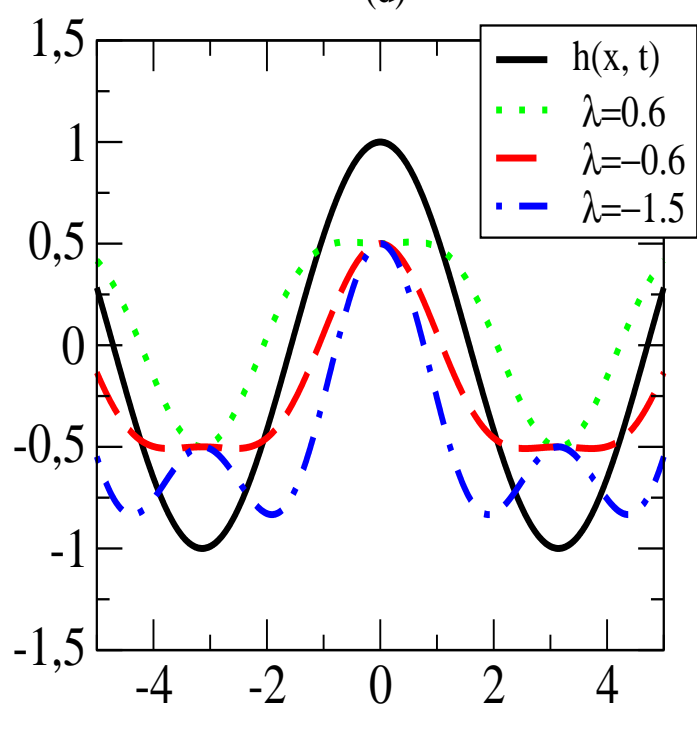

Figure 3.2: Plots of $h(x, t+\delta t)$ versus $x$, to see the effects (local) of each term of eq. 3.5 on an arbitrary surface. The black lines represent the initial, $\delta t=0$, profile $h(x, t)$. (a): $\lambda=K=0$. (b): $\nu=0.4$. (c) and (d): $\nu=0.4, K=0.1$. 
lation, reflection etc. Noise arises from the stochastic nature of local surface modification, as a result of random collisions of atoms in the near surface, induced by the ion bombardment; and makes the exact $\delta h$ in a time interval $\delta t$ non-deterministic. Finally, these terms either increase or reduce the surface width, as may be easily inferred from our discussion, as well as from Fig. 3.2 .

\subsection{Linear Theory}

In the seminal theory of P. Sigmund on ion-beam sputtering of amorphous and poly-crystalline targets, it was shown that the spatial energy distribution $E(\mathbf{r})$ of an impinging ion may be approximated by a two-dimensional Gaussian of widths $\sigma$ and $\mu$, parallel and perpendicular to the ion beam direction, respectively. Using this gaussian distributed kinetic energy [40, 41],

$$
E(\mathbf{r})=\frac{\epsilon}{(2 \pi)^{3 / 2} \sigma \mu^{2}} \exp \left(-\frac{[z+a]^{2}}{2 \sigma^{2}}-\frac{x^{2}+y^{2}}{2 \mu^{2}}\right),
$$

of an impinging ion of total energy $\epsilon$ with average penetration depth $a$, the normal erosion velocity $v=-\partial_{t} h$ at the point of impact, may be computed [42, 39] as

$$
v=p_{m} \int_{R} d \mathbf{r} \Phi(\mathbf{r}) E(\mathbf{r}) .
$$

Where the integral is taken over the region $R$ of all the points at which the deposited energy contributes to the total power $\epsilon$ of all the ions that strike the impact point within the range of the distribution. $p_{m}$, which depends on the surface binding energy and scattering cross-section, is a proportionality constant between power deposition and rate of erosion. $\Phi(\mathbf{r})$ is a local correction to the uniform flux $J_{f}$.

This provided the starting point upon which almost all the theoretical analysis of the morphology of sputtered surfaces, are based. The linear theory of Bradley and Harper (BH) is the first analytic attempt in this direction, and it is quite successful in providing an explanation of the physics behind the peculiarities of ion beam sputter erosion.

\subsubsection{Bradley-Harper Theory}

According to the $\mathrm{BH}$ theory, the time evolution of the interface height $h(\mathbf{r}, t)$ is given by the following linear deterministic equation,

$$
\partial_{t} h=-v_{0}+v_{0}^{\prime} \partial_{x} h+\zeta_{x} \partial_{x}^{2} h+\zeta_{y} \partial_{y}^{2} h-D \nabla^{2} \nabla^{2} h ;
$$


provided that there is no redeposition of eroded material, negligible reflection of ions at grazing incidence [4], and that the local slopes are small enough for non-linear effects, and shadowing effects, to be insignificant. Except for the last term, all the other terms in (3.8) arise from (3.7); i.e. since $\delta_{t} h=-v$. The last term arises from the continuity equation for diffusion of surface atoms (described in detail in section 4.2). $v_{0}(\psi)$ (a function of the local angle of incidence $\psi$ of the ion) is the erosion velocity of a flat surface; and gives an estimate of the speed of the surface while the solid is being eroded, i.e, $h(x, y, t)=h_{0}-v_{0} t$ ( $h_{0}$ is the initial height of the surface). $\zeta_{x}=J_{f} a Y_{o}(\psi) \Gamma_{x} / n=c \Gamma_{x}$ and $\zeta_{y}=c \Gamma_{y}$ are effective surface tensions, arising from the erosion process. $c=J_{f} a Y_{0}(\psi) / n, Y_{0}$ is the sputter yield of a flat surface, and $n$ is the number of atoms per unit volume in the amorphous solid. $\Gamma_{x}(\psi)$ and $\Gamma_{y}(\psi)$ are dimensionless parameters which describe the curvature dependence of the sputter yield. $D$ is the surface diffusion constant induced by thermal diffusion. A shortcoming of (3.8) is that the coefficients are expressed in terms of the local impact parameters instead of experimental parameters, e.g, $\psi$ instead of $\theta$; this is remedied in [42, 39], and discussed below.

If we consider, in $\mathbf{k}$-space a periodic perturbation, with wave vector $\mathbf{k}=$ $\left(k_{x}, k_{y}\right)$, of the form

$$
h(\mathbf{k}, t)=-v_{0} t+h_{A} \exp [i(\mathbf{k} \cdot \mathbf{r}-\omega t)],
$$

where the amplitude

$$
h_{A}(t)=h_{A}(0) \exp (R t),
$$

then (3.8) gives

$$
R=-\zeta_{x} k_{x}^{2}-\zeta_{y} k_{y}^{2}-D\left(k_{x}^{2}+k_{y}^{2}\right)^{2}, w=-v_{0}^{\prime} k_{x}
$$

i.e, since $\nabla^{2} \nabla^{2} h=\left(k_{x}^{4}+k_{x}^{2} k_{y}^{2}+k_{y}^{2} k_{x}^{2}+k_{y}^{4}\right) h_{A} \exp [i(\mathbf{k} \cdot \mathbf{r}-\omega t)]$. For $R>0$, the amplitude grows exponentially with time, hence the wave vector $\mathbf{k}^{*}$ observed experimentally is the one for which $R$ is maximum, i.e, for which $\partial_{k} R=0$, and $\partial_{k}^{2} R<0$. Below some critical angle $\psi_{c}$ both $\zeta_{x}$ and $\zeta_{y}$ are negative and in the absence of the surface diffusion term, there is no characteristic length scale (since $k^{*}=0$ or $\lambda^{*}=\infty$ ). Whereas an interplay with the diffusion term $(D>0)$ exibits a characteristic lengthscale $\lambda^{*}=2 \pi / k^{*}$, where

$$
k^{*}=\sqrt{\frac{\left|\zeta_{x / y}\right|}{2 D}}
$$

$\left(k=|\mathbf{k}|=\left|k_{x} \hat{x}\right|\right.$ or $\left.\left|k_{y} \hat{y}\right|\right)$. Suppose $\phi$ is the angle between the wave-vector $\mathbf{k}$ and the ion beam projection (which is here assumed to be parallel to the 
$x$-axis), then, $k_{x}=k \cos \phi$ and $k_{y}=k \sin \phi$. And we may re-write the ripple amplification rate $R$ (3.11), as

$$
R(\phi)=-\left(\zeta_{x} \cos ^{2} \phi+\zeta_{y} \sin ^{2} \phi\right) k^{2}-D k^{4} .
$$

We see that for $R>0$ only two orientations are possible, corresponding to the maximum of $R(\phi)$. That is, since $R(\phi)$ is either maximum when $\phi=0$ $(\mathbf{k}=k \hat{x})$ if $\left|\Gamma_{x}\right|>\left|\Gamma_{y}\right|$, in which case $R(\phi)$ decreases from $\left|\Gamma_{x}\right|$ to $\left|\Gamma_{y}\right|$ as $\phi \rightarrow 90^{\circ}$; or when $\phi=90^{\circ}(\mathbf{k}=k \hat{y})$ if $\left|\Gamma_{y}\right|>\left|\Gamma_{x}\right|$, in which case $R(\phi)$ decreases from $\left|\Gamma_{y}\right|$ to $\left|\Gamma_{x}\right|$ as $\phi \rightarrow 0$.

Note that if the curvature dependence of the sputter yield is neglected, $\Gamma_{x}=\Gamma_{y}=0$ which implies $\zeta=0$ and $R<0$, i.e, ripple formation is not possible. Thus, ripple formation is due to the curvature dependence of the sputtering yield (captured by the coefficients $\Gamma(\psi)$ ), whereby troughs are preferential eroded to crests which makes the surface unstable against periodic perturbation, with a consequent exponential growth of the amplitude. Note also that the second term on the RHS of (3.8) arises from variations (due to changing local incidence $\psi$ ) in the tilt-dependent erosion velocity along the $x$-direction, which is parallel to the projection of the ion beam direction onto the surface plane; and leads to the non-zero $w$ in (3.11), along the $x$-direction. In other words, due to the broken symmetry when tilting the ion beam 22 at an angle $\theta$ to the surface normal, in the $x-z$ plane, the ripples with $\mathbf{k}=k_{x} \hat{x}$ should propagate along the positive (negative) $x$ direction if $v_{0}^{\prime}$ is positive (negative).

Although Eq. (3.8) gives a satisfactory explanation of ripple formation, as well as a correct prediction of the ripple wavelength and orientation, it fails to explain a number of experimental features, such as the saturation of the ripple amplitude [44, 45, 46], the observation of rotated ripples [32, and the appearance of kinetic roughening [4, 47]. These shortcomings are known to be remedied with inclusion of non-linear effects into the theory.

\subsubsection{Theory of Makeev, Cuerno, and Barabási}

Makeev et. al. mapped a calculation of the coefficients, that characterize the topography evolution during sputter-erosion, from the local coordinate frame to the laboratory reference frame. They also considered higher(third)-order linear terms, and the shot noise arising from the random arrival of the ions on to the surface plane. With these calculations, they proposed the following stochastic, linear, partial differential equation for the time evolution of the surface height,

$$
\begin{array}{r}
\partial_{t} h=-v_{0}+\gamma \partial_{x} h+\nu_{x} \partial_{x}^{2} h+\nu_{y} \partial_{y}^{2} h+\Omega_{x} \partial_{x}^{3} h+\Omega_{x y} \partial_{x} \partial_{y}^{2} h \\
-K \nabla^{4} h+\eta(x, y, t) .
\end{array}
$$


Where the coefficients are explicitly given in Ref. 39, and $\eta(x, y, t)$ represents the randomness resulting from the stochastic nature of ion arrival to the surface,

$$
\left\langle\eta(\mathbf{x}, t) \eta\left(\mathbf{x}^{\prime}, t^{\prime}\right)\right\rangle=D^{\prime} \delta\left(\mathbf{x}-\mathbf{x}^{\prime}\right) \delta\left(t-t^{\prime}\right), \quad\langle\eta(\mathbf{x}, t)\rangle=0,
$$

i.e, uncorrelated both in space and time, and with zero mean. The amplitude of the fluctuations, $D^{\prime}$, is directly proportional to the average incoming particle flux. Thus, here one can obtain a continuum theoretical explanation of the experimentally observed topographies by plotting the coefficients using the experimental parameters.

Note that in (3.14) the higher (third) order linear terms merely serve as corrections to the ripple propagation velocity, which, following our treatment in section 3.4.1, leads to

$$
\omega=-\gamma k_{x}+\Omega_{x} k_{x}^{3}+\Omega_{x y} k_{x} k_{y}^{2}
$$

and have no further effect on the surface morphology. In general, odd order terms only contribute to the surface translation. (3.16) implies that, for small incidence angles, ripples propagate parallel to the ion beam direction with velocity

$$
v_{r}\left(\lambda_{x}, \lambda_{y}\right)=-\gamma+12 \pi^{2} \frac{\Omega_{x}}{\lambda_{x}^{2}}+4 \pi^{2} \frac{\Omega_{x y}}{\lambda_{y}^{2}},
$$

that is, it also depends on the most relevant Fourier mode perpendicular to ion beam direction. However, for the isotropic case the coefficients $\Omega_{x}$ and $\Omega_{x y}$ are negligible, if $\theta<60^{\circ}$, compared to $\gamma$ [39]; thus the main contribution to the ripple velocity comes from the $\gamma \partial_{x} h$ term. This implies that the interface morphology is exactly as explained in the $\mathrm{BH}$ equation in section 3.4.1. except that we now have coefficients that can be calculated directly from the experimental parameters, i.e, $v_{0}^{\prime} \rightarrow \gamma, \zeta \rightarrow \nu$, and $D \rightarrow K$, so that

$$
R=-\nu_{x} k_{x}^{2}-\nu_{y} k_{y}^{2}-K\left(k_{x}^{2}+k_{y}^{2}\right)^{2} .
$$

Thus only the surface tension coefficients determine the surface topography, and their interplay gives rise to the phase diagram shown in Fig. 3.3. In this figure, ripples oriented perpendicular to the ion beam direction are formed in region I, while ripples oriented parallel to the ion beam direction are formed in region II.

When evaluating contributions from higher-order derivative corrections to (3.14) and from the inclusion of the first-derivative component resulting from radiation-induced viscous flow in Ref. [48, it was shown that higher than fourth-order processes only weakly modify ripple parameters. The inclusion 


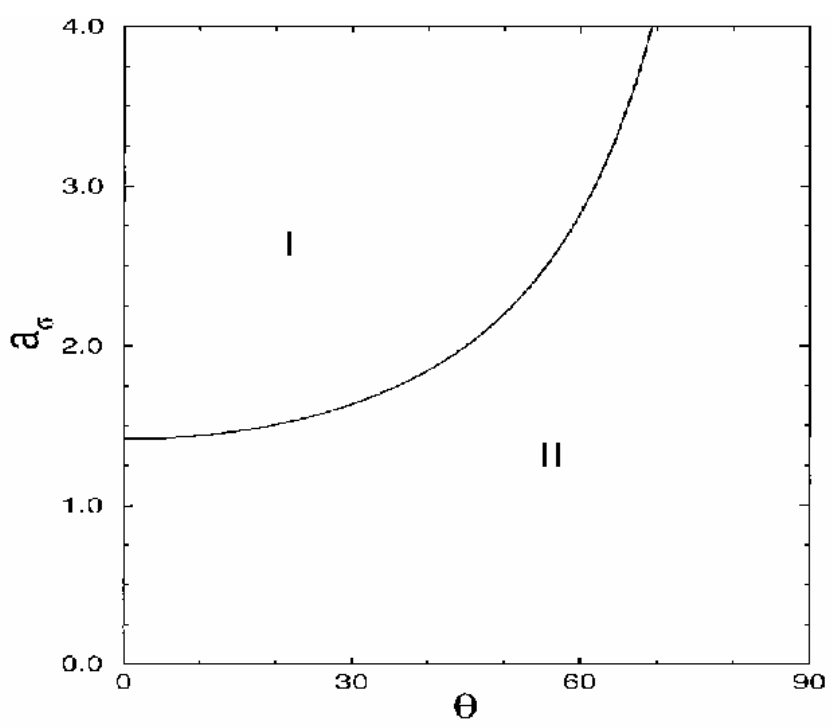

Figure 3.3: Ripple orientation phase diagram for the isotropic case $\sigma=\mu=1$, in the linear regime. Region I: $\nu_{x}<\nu_{y}<0$; region II: $\nu_{x}>\nu_{y}$. (After Makeev et. al. [39]).

of radiation-induced viscous flow is also of weak influence, though it leads to a much larger reduction in growth rate, but can attain a critical value that inhibits any ripple formation; particularly for small ion-incidence angles and energies. The inclusion of viscous-flow relaxation, however, is only relevant in radiation-amorphized materials.

\subsection{Non-linear Theory}

In the preceeding section, the interface height evolution has been described as a linear anisotropic function of the mean local slopes, in which the height fluctuations increase exponentially with the sputtering time. But, as already mentioned, some experiments have reported a saturation of the ripple amplitude, as well as the observation of kinetic roughening at long sputtering times. The correction for these limitations is discussed in the next section, where the time evolution of the interface height is seen to also depend on nonlinearities inherent in the system. But before discussing these corrections, we shall first discuss the Kardar-Parisi-Zhang (KPZ) equation, a paradigm of interface propagation, which will come up quite often in subsequent sections. 


\subsubsection{The Kardar-Parisi-Zhang Equation}

Although the KPZ equation was originally proposed as a systematic analytic treatment of the static and dynamic fluctuations of growing interfaces (e.g, eden growth model, ballistic aggregation etc.) [49] or propagating fronts (e.g, flame front, fluid flow through porous media), there are strong indications that it is applicable to the treatment of sputtered surfaces as well; at least at long time-scales. According to the KPZ equation,

$$
\partial_{t} h=\nu_{p} \nabla^{2} h+\frac{\lambda_{p}}{2}(\nabla h)^{2}+\eta,
$$

interface growth is isotropic and stable in the small slope regime, since, in the first term on the RHS, the surface is relaxed by the same amount $\nu>0$ along the $x$ and $y$ directions. The noise term $\eta$ is as defined in Eq. (3.15).

As local surface slopes increase, non-linearities become important and are responsible for the unusual properties of the interface. Note that if $\lambda_{p}=0$, (3.19) reduces to

$$
\partial_{t} h=\nu_{p} \nabla^{2} h+\eta
$$

which is known as the Edwards-Wilkinson (EW) equation. The finite size fluctuations of an interface governed by the EW equation is characterized by the exact exponents [1]

$$
\alpha=\frac{2-d}{2}, \beta=\frac{2-d}{4}, z=2 .
$$

If $\nu_{p}=\lambda_{p}=0$ in (3.19), we get the random deposition model: $\partial_{t} h=\eta$; in this case, $\alpha$ is undefined (i.e, $\alpha=\infty$ ), and $\beta=1 / 2$.

The exact scaling exponents of the KPZ equation are known only in $1+1$ dimension, where $\alpha=1 / 2, z=3 / 2$; i.e, $\alpha$ and $\beta$ satisfy the scaling relation $\alpha+z=2$. The non-linear coupling $\lambda_{p}$ is expected to be irrelevant at higher spatial dimensions (i.e, $d \geq 3$ ), due to the existence of a critical dimension at $d=2$ in which the non-linear coupling is only marginally relevant, and in which direct numerical simulations may be hampered by a large crossover regime [49]. Simulations in $2+1$ dimensions yield $z$ in the neighborhood of 1.7 [1. Due to this critical dimension, phase transitions are possible between different universality classes. That is, since the irrevance of $\lambda_{p}\left(\lambda_{p} \simeq 0\right)$ results to the EW universality class; and, the irrelevance of both coefficients $\left(\nu_{p}=\lambda_{p}=0\right)$ result to the random deposition universality class.

Assuming a quenched noise, by supposing that the sample surface is very rough initially, then the effects of shot noise (or annealed noise) in (3.19) 
may be neglected for all but the longest times [50, leaving us with the deterministic KPZ equation. Using scaling arguments, it was shown in Ref. 51] that if the initial roughness scales as $L^{\alpha_{0}}$ then

$$
z=\min \left(2,2-\alpha_{0}\right) \text { and } \alpha=2-z,
$$

i.e, $\alpha=\min \left(0, \alpha_{0}\right)$. This implies $z$ has an upper bound of 2 , below which it depends on $\alpha_{0}$ as $z=2-\alpha_{0}$; and $\alpha=0(z=2)$ for $\alpha_{0}>0$. But these predictions have not yet been tested in 2 and higher spatial dimensions.

\subsubsection{The Kuramoto-Sivashinsky Equation}

According to Cuerno and Barabási (CB) 42], the limitations of the BH model can be corrected by the inclusion of noise and non-linear terms to 3.8. In other words, the time evolution of a sputtered surface is described by the noisy Kuramoto-Sivashinsky equation (KS) [52],

$$
\begin{array}{r}
\partial_{t} h=-\left|\nu_{x}\right| \partial_{x}^{2} h-\left|\nu_{y}\right| \partial_{y}^{2} h+\frac{\chi_{x}}{2}\left(\partial_{x} h\right)^{2}+\frac{\chi_{y}}{2}\left(\partial_{y} h\right)^{2} \\
-K \nabla^{2} \nabla^{2} h+\eta .
\end{array}
$$

i.e, assuming a reference frame moving with velocity $v_{0}$ along the vertical. The non-linear coefficients $\chi_{x}$ and $\chi_{y}$ describe the tilt-dependent erosion rate, in analogy with the nonlinear coefficient in the Kardar-Parisi-Zhang (KPZ) equation. The KS equation has previously been used to model flame front (interface) propagation and diffusive instabilities of chemical waves. The linearized KS equation has a band of unstable modes with wave vector smaller than a threshold value. In the isotropic KS equation $\left(\nu_{x}=\nu_{y}=\nu\right.$; $\left.\chi_{x}=\chi_{y}=\chi\right)$, an interplay between the linear instability $\nu$ and the non-linear coupling $\chi$ between the unstable modes leads to a state of chaos is space and time 53, 50.

Applied to sputtering phenomena Eq. (3.23) is known as the CuernoBarabási (CB) continuum model. They explicitly calculated the coefficients in terms of physical (experimental) parameters characterizing the sputtering process, for $\sigma=\mu$. When fully developed, the surface has a cellular structure, where each cell is either a rounded protrusion on the surface $(\chi>0)$ or a rounded hole $(\chi<0)$. These cellular structures are not periodic in space and time, but rather, appear and disappear chaotically with time. Using the isotropic, noisy KS equation, it was shown in Ref. [50] that the mean cell width scales as $(K /|\nu|)^{1 / 2}$, and the mean cell height scales as $|\nu| /|\chi|$. And, since both $\nu$ and $\chi$ are proportional to the ion flux $J_{f}$ and are independent of the sample temperature $T$, the mean cell width does not depend on $J_{f}$ or $T$. 


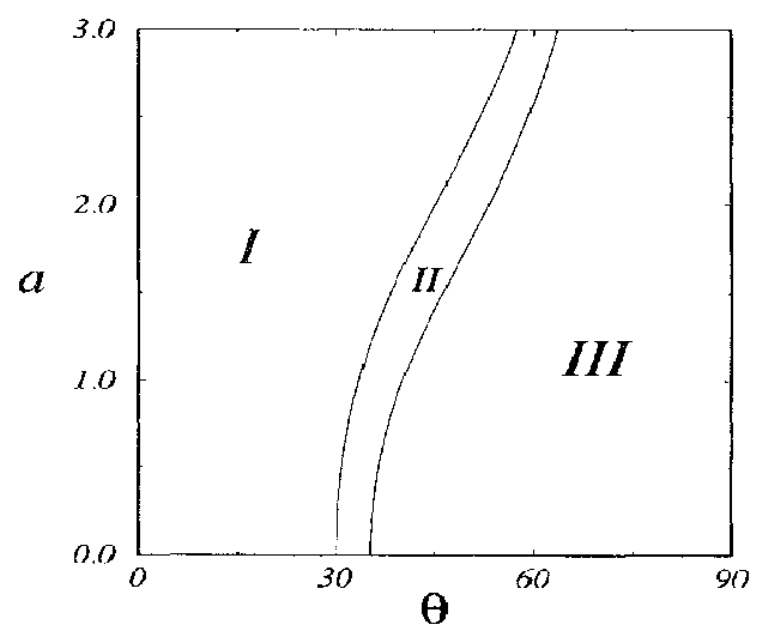

Figure 3.4: Ripple orientation phase diagram for the isotropic case $\sigma=\mu=1$, in the nonlinear regime. Region I: $\nu_{x}<0, \nu_{y}<0, \chi_{x}<0, \chi_{y}<0$. Region II: $\nu_{x}<0$, $\nu_{y}<0, \chi_{x}>0, \chi_{y}<0$. Region III: $\nu_{x}>0, \nu_{y}<0, \chi_{x}>0, \chi_{y}<0$ (After Cuerno and Barabási [4]).

As shown in Fig. 3.4, Cuerno and Barabási found the existence of three phases, with boundaries defined by $a$ and $\theta$; and with the scaling behaviour dependent on the relative sign of $\nu_{x}, \nu_{y}, \chi_{x}$, and $\chi_{y}$. Note that $\nu_{y}<0, \chi_{y}<$ 0 for $0 \leq \theta \leq \pi / 2$.

If the diffusion is isotropic, and the characteristic lengthscale $\lambda_{c}=\sqrt{K /|\nu|}$ that arises as a result of the competition and interplay between the negative surface tension and surface diffusion is of the order of the lengthscale of the system $l$ (i.e $l \sim \lambda_{c}$ ), the nonlinear terms and noise play very little or no role in the surface morphology, and the surface conforms to the $\mathrm{BH}$ linear theory, such that a ripple morphology dominates, with ripples oriented along the direction ( $\mathrm{x}$ or $\mathrm{y}$ ) which presents the largest absolute value for its surface tension coefficient(i.e ripples are expected in regions I, II and III for all theta). The (anisotropic) large lengthscale behavior $l>>\lambda_{c}$ is expected to be different, since both nonlinearities and noise may now be relevant; consequently, they discussed some possible scenarios, based on the result availlable in the literature for some of its limits, as follows.

One may expect both $\nu_{x}$ and $\nu_{y}$ to change to positive values in a renormalization group analysis of the KS equation in $2+1$ dimensions [42. Indeed, it is well established that in $1+1$ dimensions, the scaling behaviour of the 
KPZ equation is the same as that of the Kuramoto- Sivashinsky (KS) equation [54, 55, 56, 57]. Though in $2+1$ dimensions, there is evidence that $\mathrm{KPZ}$ and KS equations are in the same universality class as well [58, but it is still unclear whether, or not, this is the case; since different groups have reported different results [59, 60, 61].

Since $\nu>0$, implies a relaxation of the surface, the surface diffusion term will then be insignificant $(K \ll 1, \nu>K)$ to the relevant surface relaxation mechanism. The scaling properties are then described by the anisotropic KPZ (AKPZ) equation. The AKPZ equation predicts algebraic scaling (surface width grows algebraically, and saturates) if $\chi_{x} \chi_{y}>0$; in which case the non-linear terms are relevant. This is the case in region I, with exponents $\alpha=0.38$ and $z=1.6$; i.e, surface is characterized by KPZ equation in $2+1$ dimensions $\left[62\right.$, 63. If $\chi_{x} \chi_{y}<0$, in which case the non-linear terms are irrelevant since they tend to cancel each other, the AKPZ equation predicts a logarithmic scaling $(\alpha=0)$; i.e, width grows logarithmically.

Where $\nu_{x}>0$ and $\nu_{y}<0$ (region III), the negative $\nu_{y}$ may change to a positive value such that the scaling is again logarithmic (AKPZ), since $\chi_{x} \chi_{y}<$ 0 . But there is no consensus as yet on the asymptotic scaling behaviour of the KS equation in $(2+1)$ dimensions [64], since direct simulations 65] have not been conclusive, and analytic treatments by different groups have led to different conclusions [58, 59].

Park et. al. [66] discussed the behavior of, or relevance and non-relevance of nonlinearities and the noise in Eq. (3.23) to be governed by the time scale of modification; where they demonstrated a clear separation between the linear and nonlinear behavior. For erosion times $t<\tau$ (crossover time), the nonlinear terms are irrelevant; ripples are formed, as predicted by the linear BH theory, with an amplitude, and hence surface width, that grows exponentially with erosion time. For $t>\tau$, the nonlinear terms stabilize the surface width, and completely determine the surface morphology. They destroy the ripple morphology generated in the linear regime, when $\chi_{x} \chi_{y}>$ 0 , and lead to a rough phase governed by kinetic roughening. While they result in a long and apparently rough transient regime, followed by a new morphology of rotated ripples, when $\chi_{x} \chi_{y}<0$. This rotated ripple phase is stable against the noise (i.e the noise term is irrelevant here).

The CB model does not predict a coarsening effect $\lambda \propto t^{z}$ in time 1 , in contrast to experiments [30, 22, 32]. It also foresees $\alpha \approx 0$ for $\theta>\theta_{c}$.

\footnotetext{
${ }^{1}$ Very recently, a continuum theory has been proposed in Ref. 68 to address this issue
} 


\subsection{Extensions}

The exact forms of the continuum theory discussed in the preceeding sections are at times not satisfactory to specific problems, which neccessitates an extension of the theory by addition of relevant terms. Such pertinent extensions are discussed in this section.

\subsubsection{The Theoretical Model of Chason et. al.}

In other to account for the quantitative difference between the $\mathrm{BH}$ theory and their experiments [11, Chason et. al. proposed the following theoretical model. The model includes the effects of relaxation via viscous flow $(F)$ to the amplification rate $R_{q}$, so that the surface spatial frequency spectrum is given by

$$
|h(\mathbf{q}, t)|^{2}=\left|h_{0}(\mathbf{q})\right|^{2} \exp \left(R_{q} t\right)+\left(\alpha_{q} / R_{q}\right)\left[\exp \left(R_{q} t\right)-1\right] .
$$

$R_{q}$ represents the balance between the relaxation rates $F q$ and $D q^{4}$ by viscous flow $(F)$ and surface diffusion $(D)$ respectively, and the structure dependent roughening rate $S q^{2}$ by sputtering $(S)$. $h_{0}(\mathbf{q})$ is the initial roughness spectrum. The preferred wave vector $q^{*}$ is the resultant of the competing processes, and occurs at the maxima of $R_{q}$ (if $S$, sputtering, dominates) or minima of $R_{q}$ (if the smoothening combination of viscous flow, and diffusion, dominates). Solving the extremal equation for the crystalline phase $(F=0)$ gives $q^{*}=(S / 2 D)^{1 / 2}$ and $R_{q}^{*}=S^{2} / 4 D . S \neq S(T)$, thus, as D increases at high temperatures, $R_{q}^{*}$ decreases and the surface roughens more slowly than at a lower temperature $T \geq 250^{\circ} \mathrm{C}$. Since $S \sim f$, where $f$ is the ion flux, the model also predicts that $R_{q}^{*}$ depends on the square of the ion flux; a behavior they have observed.

\subsubsection{Normal Incidence Quantum Dot Formation}

A quantum dot (QD) is a particle of matter so small (e.g of nanometer dimensions) that the addition or removal of an electron changes its properties in some useful way. Thus, their fabrication and physical properties are of interest due to their potential applications in nanotechnology, in particular, optoelectronics. It was demonstrated by Facsko and collaborators [25] that ion beam sputtering $\left(\mathrm{Ar}^{+}\right.$on crystalline $\mathrm{GaSb}$ ) is a more attractive alternative to two previously used methods of quantum dot manufacture: Epitaxy; and lithographic techniques of spontaneous growth of semiconductor QDs in the Stranski-Krastanov growth mode. The sputtering method is cost effective, and can produce small, high density dots, with a uniform size 
distribution. It was also demonstrated, simultaneously, by Rusponi and collaborators [38, 69] that such normal incidence sputtering [of metallic copper $(\mathrm{Cu})$ and silver $(\mathrm{Ag})$ surfaces] produces uniform depressions or holes.

Kahng et. al. [70] have shown that the formation of ordered QDs instead of the random hillocks and depressions predicted by the linear theory, and observed in some experiments, is due to the relevance of non-linear terms. Assuming that ion-induced effective diffusion and thermally activated surface diffusion are the main relaxation mechanisms [71, the following equation was proposed for the height evolution of the sputtered surface,

$$
\begin{array}{r}
\partial_{t} h=\nu_{x} \partial_{x}^{2} h+\nu_{y} \partial_{y}^{2} h+\frac{\chi_{x}}{2}\left(\partial_{x} h\right)^{2}+\frac{\chi_{y}}{2}\left(\partial_{y} h\right)^{2} \\
-K_{x} \partial_{x}^{4} h-K_{y} \partial_{y}^{4} h-K_{x y} \partial_{x}^{2} \partial_{y}^{2} h+\eta
\end{array}
$$

Under normal incidence, the coefficients in this equation are isotropic, and there is rotational symmetry, thus the equation that guides the height evolution of sputtered surfaces at normal incidence, may be written as

$$
\partial_{t} h=\nu \nabla^{2} h+\frac{\chi}{2}(\nabla h)^{2}-K \nabla^{4} h+\eta,
$$

where $\nabla=i \partial_{x}+j \partial_{y}, \nabla^{2}=\nabla \cdot \nabla=\partial_{x}^{2}+\partial_{y}^{2},(\nabla h)^{2}=\nabla h \cdot \nabla h=\left(\partial_{x} h\right)^{2}+\left(\partial_{y} h\right)^{2}$, $\nu_{x}=\nu_{y}=\nu, \chi_{x}=\chi_{y}=\chi, K_{x}=K_{y}=K$, and $K_{x y}=K_{x}+K_{y}=2 K$.

The ion-induced effective diffusion is dominant at low temperatures, while thermal diffusion is dominant at high temperatures. The coefficients are given in Ref. [70. The quenched form of (3.26) was used in their numerical integration, in order improve the uniformity of the dots. The noiseless (save for the quenched noise, in the random initial surface configuration) integration reveals the non-linear nature of the QDs.

As shown in Fig. 3.5, in the early stages [Fig. 3.5 (a) and (d)], when non-linearities are irrelevant, the surface is dominated by small wavy perturbations (or non-oriented, due to the isotropy, short ripples) generated by the interplay between the ion-induced instability and surface relaxation. After some characteristic time $\tau$, these structures turn into isolated by closely packed islands, if $\chi>0$ [Fig. 3.5 (b)], or holes [Fig. 3.5 (e)], if $\chi<0$. At times $t \gg \tau$, the non-linear terms lead to kinetic roughening of the surface at large length scales [42, 70, and while the QDs or holes do not disappear [Fig. 3.5 (c) or (f), respectively], the substrate on which they exist becomes rough, destroying the overall island/hole uniformity and ordering. This loss of uniformity is shown in the increased width of the island height distribution at later times, implying a higher fluctuation in the island height at later times.

The crossover time $\tau$ can be monitored, through the surface width, as the crossover time from the regime of the exponential growth of the surface 
(a)

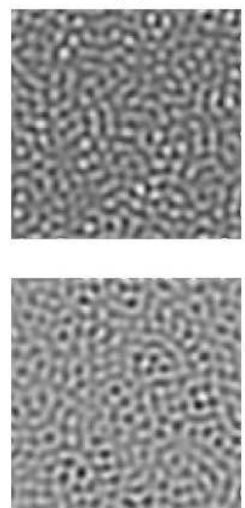

(d) (b)

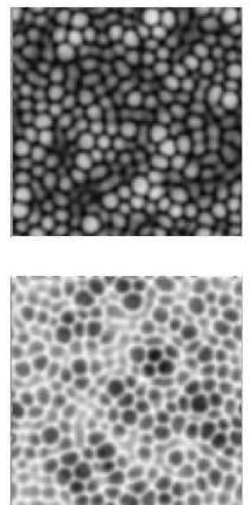

(c) (c)
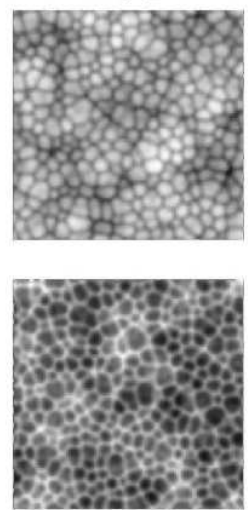

(f)

Figure 3.5: (a) - (c): Surface morphologies predicted by Eq. (3.26), for $\chi=1$ at different stages of surface evolution. The pictures correspond to $t=4.0,5.8$, and $8.0 \times 10^{4}$, respectively. (d) - (f): The same as in (a) - (c), but for $\chi=-1$. (After Park et. al. 66]).

roughness, to the regime of the steady-state roughness; since it has been demonstrated 66] that the non-linear terms are responsible for the saturation of the surface roughness. Thus, ordered and uniform QDs, or holes, can be obtained only at the crossover between the linear and non-linear regimes, i.e, at $t \simeq \tau$; and the dot size, though independent of the flux and temperature, may be tuned by changing the ion energy.

\subsection{Theory of Simultaneously Rotated and Sputtered Surfaces}

As mentioned earlier, ripple formation is a nuisance in a number of surface processing and analysis applications (e.g SIMS, AES). In this case the problematic ripples can be prevented from forming, or removed by Zalar rotation 28; ; a procedure in which the sample is simultaneously rotated as it is being sputtered. Such concurrent rotation and sputtering leads, in many cases, to remarkably smooth surfaces [72, 29]. In some instances, simultaneous rotation and sputtering still roughens the surface, albeit at a slower rate than without rotation. A theoretical understanding of the former case is provided in the next section (3.7.1), from the continuum theory; and that of the latter 
case is discussed in section 3.7 .2 .

\subsubsection{Pattern Suppression, Smoothening, and Roughening}

If the projection of the ion-beam is inclined at $\phi_{i}$ to the $x$-axis, as is the case for a rotating substrate, and the wave vector $\mathbf{k}$, of some Fourier mode, is inclined at $\phi_{k}$ to the $x$-axis, then in Eq. (13.13) $\phi \rightarrow \phi_{k}-\phi_{i}$. If we include an additional term $F$ for viscous flow [11, 8], Eq. (3.13) becomes

$$
R_{v 0}=-F k-\left[\zeta_{x} \cos ^{2}\left(\phi_{k}-\phi_{i}\right)+\zeta_{y} \sin ^{2}\left(\phi_{k}-\phi_{i}\right)\right] k^{2}-D k^{4}
$$

where $F=\gamma_{s} /\left(2 \mu_{v}\right)(F \geq 0), \gamma_{s}$ is the surface tension, and $\mu_{v}$ is the viscocity (as $\mu_{v} \rightarrow \infty, F \rightarrow 0$ ). Though $R_{v 0}=R-F k, R_{v 0}$ may still be positive and ripple formation possible in the presence of the enhanced smoothening due to ion bombardment induced viscous flow, provided that $F<F_{c}$; where $R-F_{c} k=0$. One may calculate $F_{c}$ by assuming that $R_{v 0}\left(F_{c}\right)=R_{v 0}\left(k_{m}\right)=0$, where $k_{m}$ is the value of $k$ for which $R_{v 0}$ is maximum (which for simplicity of calculation we here assume to be zero). Since we are already familiar with the maximum amplitude growth rate occuring along a direction parallel or perpendicular to the ion beam projection, and recalling that $\zeta=c \Gamma$, we may re-write (3.27) as

$$
R_{v}=-F k+c|\Gamma| k^{2}-D k^{4},
$$

which implies

$$
F_{c}=c|\Gamma| k-D k^{3} .
$$

Thus $\partial_{k} R_{v}\left(F_{c}\right)=0$ yields

$$
k_{m}=\sqrt{\frac{c|\Gamma|}{3 D}}
$$

i.e,

$$
F_{c}=c|\Gamma| \sqrt{\frac{c|\Gamma|}{3 D}}-D\left(\sqrt{\frac{c|\Gamma|}{3 D}}\right)^{3}=2 D\left(\frac{c|\Gamma|}{3 D}\right)^{3 / 2} .
$$

Thus for ripple formation to occur on materials with low viscocity $F$ must be less than $F_{c}$, since $R_{v} \leq 0$ if $F \geq F_{c}$, where $F_{c}$ is given by Eq. (3.31)

Considering Eqs. (3.8) and (3.9), if we assume a reference frame moving with velocity $v_{0}$ along the erosion direction, we have

$$
\partial_{t}|h(\mathbf{k}, t)|=R_{v 0}|h(\mathbf{k}, t)| \text {. }
$$


which has the solution $|h(\mathbf{k}, t)|=|h(\mathbf{k}, 0)| \exp \left(R_{v 0} t\right)$. Since keeping the ion beam direction fixed and rotating the substrate is equivalent to keeping the substrate fixed and rotating the ion beam, sample rotation is introduced into the theory [73] by letting $\phi_{i}$ evolve with time as $\phi_{i}(t)=\omega t+\phi_{i}(0)=\omega t$; i.e, assuming that the initial $\phi\left(\phi_{i}(0)\right)$ is zero. So that [3],

$$
|h(\mathbf{k}, t)|=|h(\mathbf{k}, 0)| \exp \left(R_{\text {rot }} t\right) \exp \left(\frac{c\left(\Gamma_{y}-\Gamma_{x}\right)}{4 \omega} k^{2} \sin \left[2\left(\omega t-\phi_{k}\right)\right]\right)
$$

where

$$
R_{\text {rot }}=-F k-\frac{c}{2}\left(\Gamma_{x}+\Gamma_{y}\right) k^{2}-D k^{4} .
$$

Thus $|h(\mathbf{k}, t)|$ either grows $\left(R_{\text {rot }}>0\right)$ or decays $\left(R_{\text {rot }}<0\right)$ exponentially with time, the second exponential factor simply superimposing a periodic oscillation on this overall increase or decrease. In other words $R_{\text {rot }}$ is the analogue of $R_{v}$ with sample rotation, and the effect of sample rotation on the ripple amplification rate is to transform $|\Gamma| \rightarrow\left|\Gamma_{\text {av }}\right|=\left|\Gamma_{x}+\Gamma_{y}\right| / 2$, which implies a second cut-off

$$
F_{c_{r o t}}=2 D\left(\frac{c\left|\Gamma_{\mathrm{av}}\right|}{3 D}\right)^{3 / 2} .
$$

$\Gamma_{x}$ and $\Gamma_{y}$ are always negative [41, 73]. Assuming $\theta<\theta_{c}$ (in which case $\Gamma_{x}<\Gamma_{y}<0$, or $\left.\left|\Gamma_{x}\right|>\left|\Gamma_{y}\right|\right)$, and suppose $F<F_{c_{\text {rot }}}$ then $0>\left(\Gamma_{x}+\Gamma_{y}\right) / 2>$ $\Gamma_{x}$. This implies $R_{\text {rot }}<R_{v 0}$ for all values of $k$, i.e, amplitude growth rate is suppressed with sample rotation, as observed in the experiments. If $F>F_{c_{\text {rot }}}$ (i.e, $0<F_{c_{\text {rot }}}<F<F_{c}$ ), $R_{\text {rot }}<0$ whereas $R_{v}>0$, which implies that the ripples that would have been produced without rotation are suppressed with rotation and a smooth surface is obtained instead; in agreement with experiments (e.g, [3, 29, 72]). If $F<F_{c_{\text {rot }}}$, there are $k$ values with $R_{\text {rot }}>0$ hence the surface roughens albeit at a slower rate than without rotation. However, even though $R_{\text {rot }}>0$ ripples can not be formed because of the rotational symmetry about the $z$-axis; the nature of the roughening in this case is influenced by non-linear corrections (i.e, as in the KS equation).

Thus, according to Ref. [73], the smoothing effects of viscous flow and surface self-diffusion can prevail over the roughening effect of curvaturedependent sputtering, when a sample is rotated while being sputtered. This leads to a smoothening of the surface, prevents ripple formation if sample is rotated from the beginning of the sputtering, or removes ripple patterns, if sample rotation begins after pattern formation. This predicts a transition from a smooth to a rough interface as the sample temperature is increased; assuming that the surface remains crystalline as it is heated to higher temperatures [73, 11]. 


\subsubsection{Topography and Dynamic Scaling}

A theory of the dynamic scaling of ion-sputtered rotating surfaces was proposed in Ref. [50]. Starting from the noiseless version of Eq. (3.23), and considering a rotating frame of reference $(X, Y, Z)$ corresponding to nonzero azimuth $\phi$ (as in rotated samples) in which the ion beam travels in the $X-Z$ plane before impact, Bradley showed that the surface height evolution is governed by the isotropic equation,

$$
\partial_{t} u=\nu_{\mathrm{av}} \nabla^{2} u-D \nabla^{2} \nabla^{2} u+\frac{1}{2} \chi \mathrm{av}(\nabla u)^{2} .
$$

where $u(x, y, t)=h(x, y, t)-h_{0}+v_{0} t . \nu_{\mathrm{av}}=\left(\nu_{x}+\nu_{y}\right), \chi_{\mathrm{av}}=\frac{1}{2}\left(\chi_{x}+\chi_{y}\right)$.

As mentioned in section (3.7.1) rotating the sample around the $z$-axis with constant angular velocity $\omega$, during sputtering, is equivalent to letting $\phi$ evolve with time as $\phi=\omega t$. When $\omega$ is small ripples can still form, with an orientation that depends on $\phi$ and rotates with the beam. But when $\omega$ is large, it is as if the sample were bombarded from all $\phi$; there is isotropy and rotational symmetry (i.e, no ripples in this case). Thus there is a maximum $\omega=\omega_{m}$ above which ripples will not be formed. Suppose we rotate at this maximum $\omega=\omega_{m}$, below which ripples can still be formed, (3.12) and (3.13) implies that the growth rate of these ripples is

$$
R_{k^{*}}=\frac{\nu_{\mathrm{av}}^{2}}{4 D}
$$

Let $A_{a}$ be the first appreciable amplitude, then (3.10) implies that the time for this to occur may be written

$$
t_{m}=\frac{4 D}{\nu_{\mathrm{av}}^{2}} \ln \frac{A_{a}}{h_{A}(0)}
$$

i.e, $t_{m} \sim D / \nu_{\text {av }}^{2}$, which implies $\omega_{m} \sim \nu_{\text {av }}^{2} / D$. Ripples are formed when $\omega<<\omega_{m}$, and are absent when $\omega>>\omega_{m}$.

Therefore, according to this theory, a simultaneously sputtered and rotated (with angular velocity $\omega>>\nu_{\mathrm{av}}^{2} / B$ ) surface has rotational symmetry. And, when surface slopes become appreciable, is either in the isotropic KS universality class if $\nu_{\mathrm{av}}<0$, or in the KPZ class if $\nu_{\mathrm{av}}>0$. As regards the topography, when non-linearities are significant and the dynamic scaling is that of the KS equation, the surface either consists of rounded protrusions (dots), if $\chi_{\mathrm{av}}>0$, or rounded basins (holes), if $\chi_{\mathrm{av}}<0$. Whereas if the dynamic scaling is that of the KPZ equation and the sputtering time is sufficiently long, the topography consists of paraboloids of revolution. In other words, the expected scaling behavior is as discussed in sections 3.5 .2 and 3.5 .1 . 
The coefficients appearing in (3.36) have been calculated in Ref. [74] where it was shown that dot formation is possible only in specific regions (e.g $\sigma<\mu$ and $a \leq \sigma$ ) of parameter space for which $\nu_{a v}<0$ and $\chi_{a v}<0$. 
Progress is possible only if we can train ourselves to think about programs without thinking of them as pieces of executable code.

Edsger Dijkstra

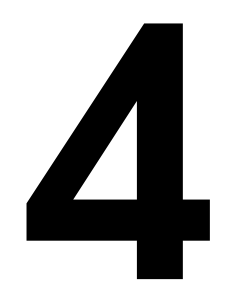

\section{Modelling for Computer Simulations}

In order to study the system at atomic length scales, where the surface is not continuous, discrete models are used. Such discrete models (e.g, as in studies of surface growth by atom deposition and aggregation) are of vital importance because they provide an essential link between the continuum theory and experiments, and, if the results are in good accord with experiments, can throw more light into the short length scale behavior of the system, and possibly also guide future experiments. As surface modification by ion bombardment is known to be governed only by the processes of sputter-erosion and surface diffusion, any model aimed at understanding the different morphologies must address these two processes.

Binary collision approximation (BCA) codes have traditionally been used as a computer simulation method for studying the effects of ion bombardment of solids, like, for instance, ranges of implanted species and damage distributions resulting from the collision cascade [75]. In the BCA, each ion trajectory is constructed as a series of repulsive two-body encounters with initially stationary target atoms, and with straight line motion between collisions. Its major limitations are that it breaks down: at low energies; when the ion has simultaneous interactions with more than one target atom; and, when collisions between moving atoms become significant. These limitations can not be ignored in this study, expecially the first one, since most surface patterns are formed from low energy sputtering.

Molecular dynamics (MD) simulation can, in principle, be applied since it has the advantage of being truly dynamical; in the sense that Newton's equations are solved to obtain the dynamical evolution of the system. However, it is quite impractical, at least in the length- and time-scales for which experimental observations are possible, due to a number of limitations: fore- 
most is the fact that MD simulations are extremely time consuming, and, even with the most sophisticated state-of-the-art supercomputers [76 one must use (e.g, in MBE) MD rates which are about 10 orders of magnitude faster than typical MBE growth rates, to speed up the simulation. Also, the typical MD simulation time is of the order of $10^{-9}-10^{-6}$ secs. [77], far below the time at which relevant surface features develop.

In addition, one needs to know the accurate interatomic potential, which is, in general not known. Though, the problem of an accurate interatomic potential maybe solved via ab-initio (or first principles, or Car-Parrinello) MD, where the forces are calculated directly, from electronic structure calculations that are performed as the MD trajectory is generated, thus considering the electronic variables as active degrees of freedom (for a review of ab-initio methods, see Ref. [8]). As a result of these limitations, MD simulations have only been used to study adatom creation, and crater formation on time scales on the order of pico-seconds $\left(10^{-12}\right.$ secs.) after ion impact [79].

Hence, in this study we only consider simulations using the Monte Carlo method. However, this approach has its limitations too, as will be seen later, but it suits our purpose considerably since the surface morphology, as a whole, is independent of the details of the dynamics of single constituents of the interface but rather, that the interactions between them either roughens or relaxes the interface. The resulting configurations can then be sampled by assigning statistical weights to them as in simulated annealing. Also, determining the overall scaling behavior of the surface might, depending on non-linearities, require simulations at (long) times outside the reach of $\mathrm{BCA}$ and MD.

\subsection{Sputtering Models}

In this section, we shall consider some discrete models of sputtering, and discuss their implementation on the computer. And in section 4.2, due to the important role of surface diffusion, we will look at different discrete models of surface migration used in computer simulations of epitaxial growth on vicinal surfaces; as well as their continuum theoretical interpretation. Any of these surface migration models can be combined with a model of the sputtering process to accurately simulate the nanometer length scale topographies arising from sputter-erosion; depending on the experimental constraints. 


\subsubsection{Cascade Model}

In this model 80, the collision cascades, arising from impinging ions, are simulated in the binary collision approximation, using a screened Coulomb potential, identical to the Krypton-Carbon (Kr-C) potential, to describe the interactions between colliding atoms. Atoms emerging, outwards from the local surface, from these cascades are allowed to sputter when their energy exceeds the surface binding energy $E_{b}=7.5 \mathrm{eV}$, derived from the heat of sublimation; redeposition of sputtered atoms is not allowed. The simulation has been done using a modification of the COSIPO (computer simulation in polycrystals) code, with the surface area limited to $L^{2}=100 \times 100$ atoms (about $20 \times 20 \mathrm{~nm}^{2}$ ), and the target divided into boxes which contain a single carbon atom, with periodic boundary conditions. Actually, the code is designed for ion energies above $1 \mathrm{keV}$ (i.e, since BCA breaks down at low energies). According to them, shadowing effects are taken into account by demanding that all collisions occur below the instantaneous surface position at the impact site.

After the completion of a collision cascade originated by each impinging ion, they simulate surface diffusion with a Wolf-Villain type model (see 4.2.2). The simulations suggest that ion bombardment creates self-affine, rough surfaces, with scaling exponents $\left[W(\langle h\rangle) \sim\langle h\rangle^{\beta^{\prime}}, W \sim L^{\alpha}\right] \alpha \simeq 0.25-0.47$, independent of relaxation mechanism, for angles of incidence in the range $0^{\circ}-60^{\circ}$, smoother surfaces (lower $\alpha$ ) corresponding to larger angles; $\beta^{\prime} \simeq 0.3$, $0.20-0.23\left(z^{\prime}=1.6-2.1\right), 0.14-0.17\left(z^{\prime}=1.9-2.6\right)$, without relaxation, with $l=1$ (nearest neighbor hopping), and with $l=2$ hopping, respectively, independent of $\theta$ [81]. These scaling exponents do not conform to any known nonequilibrium growth model.

\subsubsection{HKGK Model}

During the sputtering process, the rate of erosion of surface material, at a point on the surface, is proportional to the average power deposited there by impinging ions of uniform flux $J_{f}$ (see section 3.4). Thus, one may simulate the sputtering process by defining the surface as a $d$-dimensional grid $(1 \leq$ $d \leq 2$ ) and then eroding surface particles with a probability $p_{e}$ proportional to the power deposited there by impinging ions. This approach, with $p_{e} \propto E(\mathbf{r})$, is referred to as the Gaussian model, due to the Gaussian form $E(\mathbf{r})$ in Eq. (3.6). In other words, it simulates directly the surface morphology arising from the spatial distribution (3.6) of the kinetic energy of an impinging ion.

This model was introduced by Hartmann, Kree, Geyer, and Kölbel 82 . to simulate sputter erosion, and a similar approach has subsequently been 
taken by other simulation studies of ion-beam sputtering (see section 4.1.3). In the next section, we shall discuss some variants of this model. All of them assume the Gaussian distributed energy of an impinging ion within the bulk of the material, such that the energy received by a substrate atom decays exponentially with increasing distance from the centroid.

\subsubsection{Combined Models}

Some models have combined the two competing processes of sputtering and surface diffusion such that either of them can occur in a single iteration. Such models are discussed as follows

\section{Stochastic Model of Cuerno et al.}

The $(1+1) D$ model of Cuerno et al. [86] is defined on a square lattice of lateral size $L$, with periodic boundary conditions in the horizontal direction, which represents a $1 D$ surface, with surface points $i(1 \leq i \leq L)$; the vertical direction being the surface heights, $h_{i}$. A site is randomly chosen and subject to erosion with probability $p$, or diffusion with probability $1-p$; both being mutually exclusive events.

The sputter yield $Y(\phi)$ may be written as [87, 88,

$$
Y(\phi)=y_{0}+y_{1} \phi^{2}+y_{2} \phi^{4}
$$

where $y_{0}, y_{1}$ and $y_{2}$ are constants, which are chosen here such that $Y(0)=0.5$, $Y(\pi / 2)=0$, and with a maximum value $Y\left(\phi_{m}\right)=1$ for $\pi / 3<\phi_{m}<4 \pi / 9$ $\mathrm{rad}$. The general requirement is that $y_{0}>0, y_{1}>0$, and $y_{2}<0$ [87. Note, due to the discrete nature and the normalization constant of 7 (no overhang), only $\phi=0,45^{\circ}, 63.4^{\circ}, 71.6^{\circ}$ are possible; e.g in both cases of Fig. 1(b) in their paper, $Y(\phi)=0.5$.

If erosion is chosen, the local angle of the ion trajectories to the surface normal, $\phi \equiv \tan ^{-1}\left[\left(h_{i+1}-h_{i-1}\right) / 2 l_{h}\right]\left(l_{h}\right.$ is a unit lattice spacing along the horizontal direction), is computed and the erosion rule is applied with probability $Y(\phi)$; i.e, if random number is greater than $Y(\phi)$, abort, else proceed to erosion rule. Erosion rule (box rule): Count the number of occupied neighbors inside a square box of size $3 \times 3$ lattice spacing centered on the chosen site $i$, and erode with probability $p_{e}$, which is proportional to the number of cells in the box [see Fig. 1(b) of [86]]. Thus, this box rule favors erosion of troughs in preference to crests, thereby creating the well-known instability. An analytic form for $p_{e}$ is given in Ref. [88].

If, on the other hand, diffusion is favored, a diffusive move of the particle $i$ to a nearest neighbor site is attempted with hopping probability (i.e, 
normalized hopping rates) $w_{i \rightarrow f}=\left[1+\exp \left(\triangle H_{i \rightarrow f} / k_{B} T\right)\right]^{-1}$, where $\triangle H_{i \rightarrow f}$ is the energy difference between the final and initial states of the move; $H=\left(J / l_{v}^{2}\right) \sum_{\langle i, j\rangle}\left(h_{i}-h_{j}\right)^{2}, l_{v}$ is a unit lattice spacing along the vertical direction, and $J$ is a coupling constant.

The continuum equation for this model has been derived in [88, using the master equation approach, based on the assumption that the interface evolution is a Markov process. It was shown that the model belongs to the isotropic KS universality class [i.e, described by (3.26)] .

\section{Variants of the HKGK Model}

In Ref. 64, sputtering and diffusion are mutually exclusive events. In each MC iteration, a surface site is randomly picked for impact $\left(\theta=0^{\circ}\right)$, and a Gaussian distribution, of width $\sigma$, centered on a point $d$ units below the impact point, selects a solid particle. If the particle selected is active, i.e if it has at least a vacant nearest-neighbour or next-nearest-neighbour site to which it can migrate, then it is ejected if sputtering is the chosen event, otherwise the iteration ends; the choice is governed by a parameter $r=$ $r_{S} / r_{D}$, where $r_{S}$ is the attempt rate for a sputtering event per site, and $r_{D}(=1)$ is the attempt rate for a diffusive event of an active particle; such that the probability of eroding the particle is $p_{S}=r N /\left(r N+N_{a}\right)$, where $N=L_{x} \mathrm{x} L_{y}$, and $N_{a}$ is the number of active particles. If diffusion is the chosen event, an active particle is randomly selected from the list of all active particles, and migration to a randomly selected vacant neighboring site is allowed/disallowed based on the metropolis algorithm. Note, if $N_{a}=0$ (flat surface), $p_{S}=1$; they have considered $r_{S}<<r_{D}$; and used $a=\sigma=1$, the value of $\mu$ used was not reported, could be $\mu=\sigma$. The temperature has been arbitrarily chosen, bearing in mind that, it must be set sufficiently low in order for the solid not to melt or evaporate; but if too low, diffusion attempts are rarely successful and the rate of thermal diffusion is reduced. Furthermore, $N_{a}$ increases with simulation time, hence sputtering becomes less likely and diffusion becomes more and more likely.

Similar to Ref. 82, an atomistic model that describes the surface as a height field $h(i, j)$, where $i, j$ denote atomized cells of a $2 D$ grid with periodic boundary conditions, was developed in Ref. [83. However, when an ion reaches the surface, they allow energy deposition with a probability that depends on the local incident angle and is defined through an approach reported elsewhere; but if energy deposition occurs, then surface atoms are eroded with a probability proportional to the Sigmund distribution, with parameters, obtained through a SRIM [84] simulation of $1 \mathrm{keV}$ Ar-ion sputtering of $\mathrm{Cu}, a=3.5, \sigma=3.0$, and $\mu=2.6$. Their surface mobility entails 
discrete jumps, from a randomly chosen site $i, j$ to a randomly chosen nearest neighbor site $k, l$, if $\left|\nabla^{2} h(i, j)\right|$ or $\left|\nabla^{2} h(k, l)\right|$ (which represents the local curvature) exceeds a given threshold value $\kappa_{\max }=3$; provided that this increases the difference $\left|\nabla^{2} h(i, j)\right|-\nabla^{2} h(k, l) \mid$.

The kinetic Monte Carlo (KMC) model in Ref. 113 uses the same sputtering mechanism as in Ref. [82, but the choice of the reference frame is different. Here, they used the same reference frame as in Ref. [39], to describe the local coordinates of the incident ion relative to the impact point; and they scaled the sputter yield for a flat surface by a factor equal to the ratio of the energy deposited on a rough surface to the energy deposited on a flat surface. Surface diffusion is described by assigning an Arrhenius type hopping rate, $\Gamma=\nu e^{-\left(E_{d}+n E_{b}\right) / k_{B} T}$, to each surface atom with an activation energy proportional to the local bond coordination; where $\nu=10^{13} \mathrm{~s}^{-1}$ is the attempt frequency, $E_{d}$ is the activation energy for adatom hopping, $n$ is the number of nearest neighbors, and $E_{b}$ is a bond energy. The effects of energy deposited by ions, on diffusion, was ignored as it is dissipated quickly, relative to the rate of any diffusive event.

\subsection{Surface Diffusion Models}

It was shown by Krug et. al. 89] that nonequilibrum conditions generically generate a surface diffusion current, which only depends on the local surface inclination $\nabla h$, and can not be derived from a generalized chemical potential as expressed in Mullins' theory of equilibrium surface diffusion. In other words, the microscopic origin of the diffusion current in out-of-equilibrium surfaces, is in the tilting (local or non-local) of the surface, $h \rightarrow h+\partial \mathbf{r} \cdot \nabla h$, which induces a preference for particles to move either uphill or downhill (see section 4.2.1). In the case of particle deposition, the height increases with each (local) additional particle, whereas in particle removal, the height decreases with each particle loss (i.e provided there is no redeposition, as might be the case in the vicinity of a crater).

Much insight, into the nature of this diffusion current of adatoms has been gained by simulations of growth by MBE. In such simulations, implementation of adatom creation via atom deposition is a trivial increment of the height of a randomly chosen $\mathbf{i}$ by one (i.e, $h(\mathbf{i}, t) \rightarrow h(\mathbf{i}, t)+1)$; which, by itself, represents a time evolution of the surface height. However, such height evolution does not conform to experimental findings; despite the inclusion of noise. Extensive research has shown that inclusion of local constraints, which allow or disallow a displacement of the adatom, from the chosen site $\mathbf{i}$, are crucial; and in fact are responsible for the resulting time evolution of the 
interface. Hence, there exists some discrete models that capture the essential physics of surface migration, where such migration is viewed as either a free or a constrained random walk of adatoms (which maybe reversible or irreversible) in search of highly coordinated positions, guided by the rule to maximize the bonding or number of the nearest neighbors.

Since surface diffusion also play a crucial role in the determination of the surface morphology of sputtered materials, the intensive study of the process in MBE simulations affords us ready-made models to be inculcated into our simulations. Hence we take a look at the relevant mechanisms of a few such models, starting with a continuum description.

\subsubsection{Continuum Description}

In discrete limited-mobility atomistic surface growth models, surface diffusion implies the migration of each adatom (additional atom), until incorporation onto the surface, according to some constraint; e.g, surface energy minimization, increase (or maximization) of local coordination, minimization of some scalar field, etc. Such constraints classify the model, with unique scaling exponents which differ in each spatial dimension. Due to the SOS constraint, growth can be described on a coarse-grained level by the continuity equation (for conserved particle current)

$$
\frac{\partial h}{\partial t}=-\nabla \cdot \mathbf{j}+F+\eta(\mathbf{x}, t),
$$

where $\mathbf{j}$ is the local diffusion current, a function of the derivatives of $h, \eta$ has the same properties as (3.15), with $D=F \Omega^{2} ; F$ is the incoming particle flux, and $\Omega=a_{\perp} a_{\|}^{d}$ is the surface cell volume with lattice constants $a_{\perp}$ and $a_{\|}$in the growth direction and in the $d$-dimensional substrate, respectively.

If we define a scalar field $K$, as in Ref. 90, which is a function of $h$ and its various differential forms $\nabla^{m} h,(\nabla h)^{n}$, where $m$ and $n$ are integers, and the acceptable form is subject to symmetry considerations, then $\mathbf{j}$ may be expressed as a gradient of the scalar field $K$; i.e,

$$
\mathbf{j}(\mathbf{r}, t)=-\nabla K
$$

where

$$
K=\kappa h-v \nabla^{2} h+\varrho(\nabla h)^{2}+\cdots .
$$

In other words there is a net current downhill for positive slope of $K$, which increases with increasing slope; or, a net current uphill for negative slope of $K$ which increases with decreasing slope. Physically, the downhill (uphill) 
current constitute a current of adatoms (vacancies). The most general form of the continuity equation (4.2) can be written as

$$
\frac{\partial h}{\partial t}=\kappa \nabla^{2} h-v \nabla^{4} h+\varrho \nabla^{2}(\nabla h)^{2}+\cdots+F+\eta,
$$

where all coefficients are positive. Eq. (4.5) describes the time evolution of the height of a growing interface with conserved growth kinetics in the asymptotic limit.

In the discrete SOS models, the adatoms are allowed to hop within a finite diffusion length (discrete points) of $l$ sites. These models fall into either of two sets: limited mobility and unlimited mobility models.

\subsubsection{Limited Mobility Models}

These are the irreversible, limited mobility, dynamical growth models in which only newly arrived particles (adatoms) can diffuse. On arrival, the adatom diffusion is instantaneous, and it is permanently incorporated into the substrate without any further possibility of motion, once it has found its final site (which choice depends on the constraints of the model, as already mentioned).

\section{Wolf-Villain (WV) Model}

In this model [91], adatom diffusion is constrained by the the need to maximize the local coordination number. A newly arrived particle is incorporated into the substrate, at the deposition site, if the local coordination number is maximum at, within a finite distance $l$ from, this site; otherwise, it is allowed to diffuse to the site with the highest coordination number within a distance $l$. Here, an incorporated adatom with lateral nearest neighbors can still diffuse, if it can find a final site with higher coordination number. This model has been claimed to asymptotically belong to the $E W$ (see section 3.5.1) universality [89].

\section{Das Sarma-Tamborenea (DT) Model}

In this model 92, a newly arrived particle sticks to the deposition site if, on deposition, it will have at least a lateral nearest neighbor; i.e, a coordination number of 2 or more, arising from the neighbor beneath it and one, or more, in-plane nearest neighbors. Otherwise, it is allowed to search, within a finite diffusion length $l$, and diffuse to a final site with higher coordination number than at the original deposition site. Note that if the deposition site 
has a coordination site of 2 , and there is a site, within a distance $l$, with a coordination number of 4 (maximum), the adatom is still incorporated at the deposition site. In other words, this model tries to increase, but not neccessarily maximize, the local coordination number, in contrast with the WV model. The $D T$ and $W V$ models, though with very similar behaviors in $(1+1) D$ simulations, are quite distinct in $(2+1) D$ systems, where they behave differently after an initial crossover 93]; with suppression of nucleation and deposition noise, via the noise reduction technique [94]. A continuum description of this model via the scalar field $K$ is 95

$$
K=-v \nabla^{2} h+\frac{\varrho}{C}\left[1-\exp \left(-C|\nabla h|^{2}\right)\right] .
$$

\section{Discrete Models for Conserved Growth Equations}

The basic idea, according to Kim and Das Sarma [90, is to design discrete growth algorithms that are replicas of the corresponding continuum growth equations on a discretized system. This will enhance an extraction of the scaling exponents of these continuum models from simulations. Comparing Eqs. (4.2) and (4.3) in the absence of particle influx, yields

$$
\left\langle\frac{\partial h}{\partial t}\right\rangle=\left\langle\nabla^{2} K\right\rangle
$$

thus $\left\langle\frac{\partial h}{\partial t}\right\rangle<0,=0,>0$, for $K$ on maxima, intermediate, and minima, respectively. Depositing particles on surfaces inevitably leads to height variations, with local maxima, intermediate and minima. If, as in Fig. 4.1, we assume one layer deposition per unit time, then in unit time, on average, 0 , 1, and 2 particles are deposited on local maxima, intermediate, and minima respectively [90]. This implies, using total derivatives for this discrete system, $\left\langle\frac{d h}{d t}\right\rangle-1<0,=0,>0$, for local surface maxima, intermediate, and minima respectively. Thus $\left\langle\frac{d h}{d t}\right\rangle-1$ is approximately proportional to $\left\langle\frac{\partial h}{\partial t}\right\rangle$. But in order to have the exact form of the continuum equation, $K$ must be computed to determine which points are the true local minima of $K$, since this will be different for different models [depending on which terms of Eq. (4.4) are relevant].

The simulation algorithm is as follows: a surface site $i$ is randomly selected, and the scalar field $K_{x}$ is computed for both the selected site $i$ and its nearest neighbors, assuming that one particle has been added on the site $x$. Then, let the incoming particle diffuse, and stick, to a randomly selected site among the sites with $K_{x}<K_{i}$. If, however, $K_{i}<K_{x}$, then the newly arrived particle is incorporated into the substrate at site $i$, instead. The derivatives are calculated by noting that for a discretized system $h(x)$ is not continuous, 


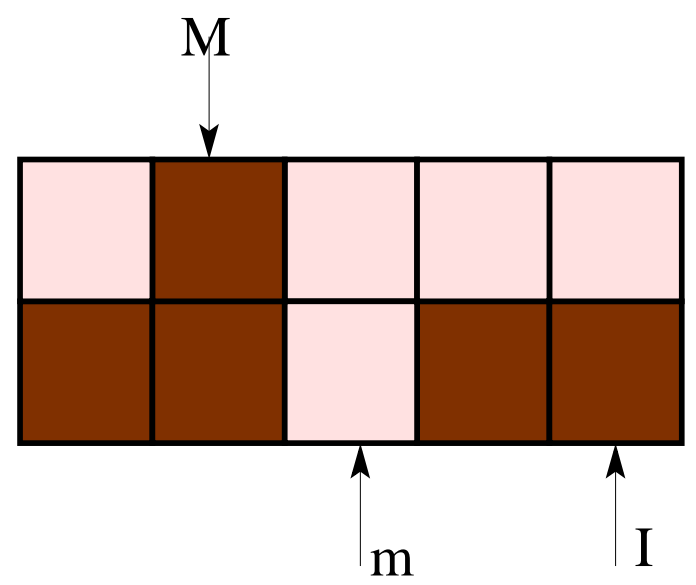

Figure 4.1: An arbirary growth profile, after a monolayer deposition in unit time. The darker colour represents the initial height profile. $M, m$, and $I$, are local maxima, minima, and intermediate points respectively.

hence the smallest increment (in the derivative) $\triangle x \rightarrow a^{\prime}$ (the lattice spacing $a^{\prime}=1$, by convention), which implies

$$
\begin{array}{r}
\nabla h=\frac{1}{2}\{[h(\mathbf{r}+\mathbf{1})-h(\mathbf{r})]+[h(\mathbf{r}-\mathbf{1})-h(\mathbf{r})]\}, \\
\nabla^{2} h=\nabla h(\mathbf{r}+\mathbf{1})-\nabla h(\mathbf{r})=h(\mathbf{r}+\mathbf{2})-2 h(\mathbf{r}+\mathbf{1})+h(\mathbf{r}) .
\end{array}
$$

Among this set are

Family Model. In this model [96, the adatoms diffuse to the local height minima sites within range $l$; thus $K=\kappa h$, and the constraint is local height minimization, or local surface energy minimization for surface hamiltonian $H \propto \triangle h$. The model is well studied and is described on a coarse-grained level by the EW equation (3.20) [97, hence the model asymptotically belongs to the EW universality class.

Larger Curvature Model. In this case adatoms diffuse to sites with minimum $K=-\nabla^{2} h$, i.e, maximum curvature [90]. The dynamical exponents associated with this model are

$$
\alpha=(4-d) / 2 ; \quad z=4 ; \beta=(4-d) / 8,
$$

Non-linear Curvature Model. In this model, $K=-v \nabla^{2} h+\varrho(\nabla h)^{2}$. Renormalization group analysis of this model by Lai and Das Sarma yield 
exponents

$$
\alpha=(4-d) / 3 ; \quad z=(8+d) / 3 ; \beta=(4-d) /(8+d),
$$

where $d$ is the substrate dimension 98.

All three cases pertain to conserved current and nonconserved noise; the noise is not conserved since particles are continually coming onto the surface from the incident flux. As regards the scaling, however, it had been pointed out in [99] (see also [100]) that several of the limited mobility models do not obey conventional scaling, and that those which do scale are expected to belong to the $E W$ universality.

In the following two sections, we shall consider the unlimited mobility surface diffusion models. In this set, all particles that are not fully coordinated, whether newly arrived or previously deposited, can diffuse by nearestneighbor hopping between deposition events. A newly deposited particle might excite a surface atom to hop into some neighboring site, hence, in the absence of this excitation, the model must ensure that the appropriate equilibrium phase is recovered; i.e, detailed balance is required. In other words, surface hopping is a reversible process in this case.

\subsubsection{The Hamiltonian Model}

Here, it is assumed that surface diffusion is controlled by the same Hamiltonian $H$ that controls the roughening of a facet in the absence of deposition (to simulate surface growth, random deposition is added to it). This is implemented on the computer by randomly selecting an adatom (which may be a newly deposited particle or a surface particle that can still diffuse) on a lattice site $i$, and allowing it to make a hopping attempt to a randomly selected nearest-neighbor site $j$, with probability $1-p$; where $p(p<1)$ is the probability that it does not attempt the hop. If it does attempt the hop, then the hopping is accepted with probability $p_{i \rightarrow j} . \quad p$ is determined from a correspondence between the ratio $(1-p) / p$ and the ratio $D / F$ of the diffusion constant of particles on a flat surface to the incoming particle flux, determined from experiments. For isotropic diffusion on terraces, with dimers as stable clusters, experiments yield $D / F=10^{6}-10^{12}$ [101]; i.e $p \sim 10^{-12}-10^{-6}$, which implies hopping attempts are amost always accepted, as expected. In order to observe the effects of kinetic roughening within reasonable time in simulations, much smaller ratios $D / F$ are used.

The Metropolis algorithm is known to preserve detailed balance, and to accurately sample, in a short time, the thermodynamic states in which a 
system spends most of its time, thus, it can be implemented, to sample the hopping history of the adatoms, with the normalized hopping rates 102

$$
p_{i \rightarrow j}=\left[1+\exp \left(\frac{1}{k_{B} T} \triangle H_{i \rightarrow j}\right)\right]^{-1}=\frac{1}{2}\left[1-\tanh \left(\frac{1}{2 k_{B} T} \triangle H_{i \rightarrow j}\right)\right],
$$

where $\triangle H_{i \rightarrow j}=H_{f}-H_{i}$ is the energy difference between the final and initial states of the move, and the surface energy is defined through the Hamiltonian of an SOS model [102, 103.

$$
H=\frac{1}{2} J \sum_{\langle i, j\rangle}\left|h_{i}-h_{j}\right|^{n}
$$

where $\langle i, j\rangle$ implies that a single term in the sum arise from two neighboring sites, and the $1 / 2$ takes care of the double counting due to index reversal in the summation (i.e, since $\langle i, j\rangle=\langle j, i\rangle$ ). The integer $n$ is unrestricted, the choice of its value depends on the required tendency of adatom diffusion.

For instance, we consider the $n=1$ and $n>1$ models in $1 D$ on a vicinal surface of large terraces separated by single steps. Such terraces (see Fig. 1 of [37]) are atomically smooth regions on the surface, and serve as the initially flat surface prerequisite to deposition, and sputtering experiments. They are obtained via ion beam treatment (bombardment) of the surface, followed by annealing by heating the sample to very high temperatures, and finally, cooling it to low temperatures.

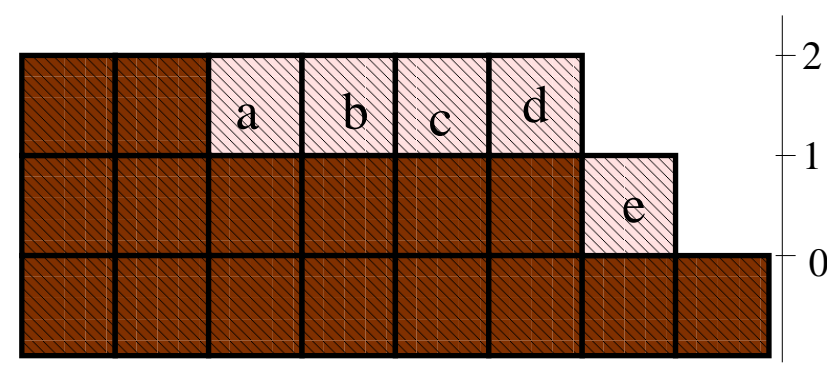

Figure 4.2: An illustration, for the Hamiltonian model in $1 D$. The light-colored, lettered, squares are possible adatom positions, as referred to in the text. The dark (brown) squares are parts of the substrate. A height scale is shown on the right.

In the following, we use the illustration in Fig. 4.2 to see the significance of $n$. Away from a step edge $(b \rightarrow c)$, diffusion is isotropic, i.e, $\triangle H=0$ and $w_{i \rightarrow j}=1 / 2$ (this is true for all $n$ ), since surface is flat. $\triangle H=-2 J$ (i.e, $\left.H_{f}-H_{i}=2 J-4 J\right)$ for a hop to an ascending step edge ( $b \rightarrow a$ in Fig. 4.2), 
and $+2 J$ (i.e, $H_{f}-H_{i}=4 J-2 J$ ) for a hop away from an ascending step edge $(a \rightarrow b)$. A hop onto an ascending step $(e \rightarrow d)$ is unfavored $\left(w_{i \rightarrow j}<1 / 2\right)$; for instance, $\triangle H=2 J$ (i.e, $H_{f}-H_{i}=4 J-2 J$ ) in the $n=1$ model, but $\triangle H>2 J$ (i.e $2^{n} J$ ) for $n>1$, with higher $n$ s requiring higher energy. In the $n=1$ model this hop requires the same amount of energy as a hop away from the ascending-step edge, whereas for $n>1$, this hop requires a higher amount of energy than a hop away from a step edge.

Thus, ascending-step edge attraction, or attachment, is strong for all $n$; but while a hop away from a step edge is equally probable in both opposing directions [step climb (higher-plane) and in-plane hop] for $n=1$, it is more favored for in-plane hop than higher-plane hop in $n>1$ models. On the other hand, a hop down a descending step $(d \rightarrow e)$ is more favored $\left(w_{i \rightarrow j}>1 / 2\right)$, as it lowers the surface energy $(\triangle H=-2 J)$. Though the surface energy is unchanged for a hop away from a descending step edge $(d \rightarrow c)$ in the $n=1$ model, it is actually preferred in the $n>1$ model. Likewise, a hop to a descending step edge $(c \rightarrow d)$ has no effect on the surface energy for the $n=1$ model, whereas it is unfavored for $n>1$.

A summary of the properties of the Hamiltonian model is as follows: in all cases, a diffusion bias occurs at step edges only, where there is a strong tendency for adatom attachment to an ascending step. For all $n$, diffusion is completely isotropic, at least two lattice spacings, away from an ascending step edge. This isotropy in diffusion extends up to a descending step in the $n=1$ model, distinct from $n>1$ which discourages hopping to a descending step edge (with decreasing likelihood of hopping as $n$ increases); i.e, for $n>$ $1(n=1)$ diffusion is isotropic up to at least two (zero) lattice spacings from a descending step. Though, a hop up an ascending step is unfavored, a hop down a descending step is favored. However, it was shown in Ref. 102 that a preference for uphill diffusion, exhibited in the ascending step edge attraction, and repulsion from a descending step edge, both of which constitute Schwoebel effect, is negative when $n=1$; absent for $n=2$; and positive for $n>2$. This slighly different scenario has been attributed to simulation configurations of lower step separation, than in the above picture, which considers configurations with widely separated steps. Nevertheless, the above argument qualitatively explains the increase of the Schwoebel barriers.

\subsubsection{The Arrhenius Model}

In this model, the hopping rate $k(E, T)$ of adatoms is taken to be of Arrhenius form 104

$$
k(E, T)=k_{o} \exp \left(-E / k_{B} T\right)
$$


where the vibrational frequency of a surface adatom $k_{o}=2 k_{B} T / \hbar, T$ is the substrate temperature, and $\hbar$ is planck's constant, and $E$ is an energy barrier to hopping. The energy barrier comprises of the energy needed to break the bond with the vertical nearest neighbor $\left(E_{v n}\right)$, i.e substrate atom immediately below the hopping atom, to break possible bonding with lateral (inplane) nearest neighbors $\left(E_{l n}\right.$, assuming a negligible next-nearest neighbor coupling), and barrier to possible step-edge diffusion (i.e, Schwoebel energy barrier $\left.E_{s e}\right)$.

In Ref. 104, the energy barriers add up to $E=E_{v n}+n_{n} E_{l n}+E_{s e}$, where $n_{n}=0, \ldots, 2 d$ is the number of in-plane nearest neighbors $(d=1,2$ is the substrate dimension). Only positive Schwoebel energy barriers are considered, and hops up are more difficult (see below) to prevent unphysical growth behaviour which otherwise result at higher temperatures due to reducing only downward interlayer transport. This model seems to be the most realistic of all surface diffusion models, since surface diffusion is an activated process, obeying Arrhenius dynamics. For instance, even if two sites are equivalent sites on a terrace, a particle must, in general, overcome an energy barrier in order to hop from one site to another.

The algorithm proposed by [104 for detecting the vicinity of a step edge is based on the expected coupling, of the hopping adatom, to the number of next nearest neighbors $\left(b_{f}\right)$ in the plane below its final site; if $b_{f}<2 \mathrm{~d}$ and the number of next nearest neighbors $b_{i}$ in the plane below the initial site is $2 \mathrm{~d}$, then there must be a lower-plane vacancy (or step edge) in the vicinity of that final site. Thus, an adatom that attempts to hop to the vicinity of a step edge, must overcome a step edge barrier, i.e, $E_{s e}>0$ if $(2 d)=b_{i}>b_{f}$, and zero otherwise. An illustration, in the $1 D$ case, is given in Fig. 4.3. The

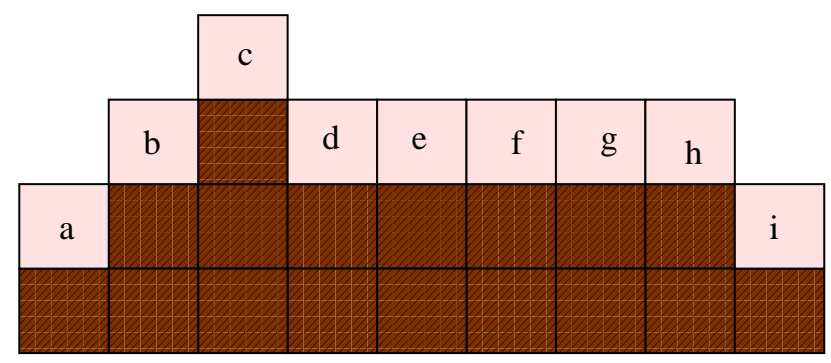

Figure 4.3: An illustration, for the Arrhenius model in 1D. The light-colored, lettered, squares are possible adatom positions, as referred to in the text. The dark (brown) squares are parts of the substrate.

SOS constraint implies $E_{v n}=k_{2}>0$ in all cases. $E_{s e}=0$ for hop $f \leftrightarrow e$ since $b_{i}=b_{f}=2$; and $b \rightarrow a$ since $b_{i}=1, b_{f}=2$. Whereas $E_{s e}>0$ for hops 
$g \rightarrow h$, since $b_{i}=2$ and $b_{f}=1 ;$ and $d \rightarrow c$ since $b_{i}=2$ and $b_{f}=0$. Note that $n_{n}=0$ for $f \rightarrow e ; n_{n}=1$ for $d \rightarrow e$ and $b \rightarrow c$. 


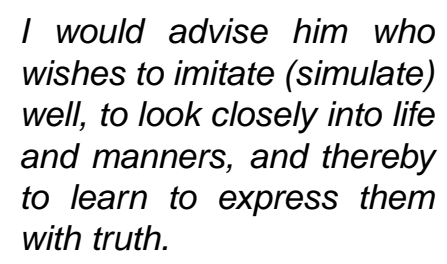

In chapter 4 we described different atomistic solid-on-solid models used in Monte Carlo simulation studies of the morphology of surfaces externally driven out of equilibrium with energetic particles. In this chapter we shall discuss how we have implemented the models applied in our study. To model surface evolution driven by ion sputtering, we have included surface roughening via sputtering and surface smoothing via surface diffusion. A simulation step consists of: (i) a sputtering attempt of surface atoms within a finite area $\left(\ll L^{2}\right)$, in the vicinity of the ion impact; and, (ii) a possible diffusion sweep. The simulation time $t$ is measured in terms of these steps (i.e. in units of the number of ions/surface atom) and corresponds to the fluence in experiments. The ratio of sputtering step/diffusion sweep is as governed by typical experimental rates. The sputtering process is simulated as a combination of ion motion and erosion of atoms [105, 106]. In our study, we have focussed on the HKGK sputter-erosion model. Our results are discussed in chapter 6. In this chapter, we mention a few details, which have not already been mentioned in chapter 4 , that are specific to our implementation of the models.

In the introduction of the HKGK model to the literature, only the erosion of the uppermost surface atoms were considered, which implies weaker nonlinear effects. Non-linear effects are known to be crucial to surface morphology, hence, later on in section 5.1.1, we account for stronger non-linearities by extending the model to include lateral erosion (see Fig. 5.31). Thus, in addition to the previous erosion of only the uppermost atoms in a multi-layered column (see Fig. [5.3), we also consider the erosion of lower surface atoms in the column; provided that they have at least a vacant lateral nearest neighbor.

The rest of the chapter is organized as follows: in section 5.1, we give 
details of our simulations using the previous version of the HKGK model. In sections 5.1.1, and 5.1.2, we discuss our extension of the model to include lateral erosion, and sample rotation, respectively. Finally, in section 5.2 we will discuss specific details of how we have implemented the surface diffusion models, as well as the values of the parameters, used in this work.

\subsection{Surface Erosion}

In our simulations of surface topography evolution using the HKGK model (described in section 4.1.2), the material surface is defined by a time-dependent discrete height function $h(x, y, t)[(2+1) \mathrm{D}$ model $]$ which is initially flat, i.e, $h(x, y, 0)=$ constant. Periodic boundary conditions are assumed on the surface plane.

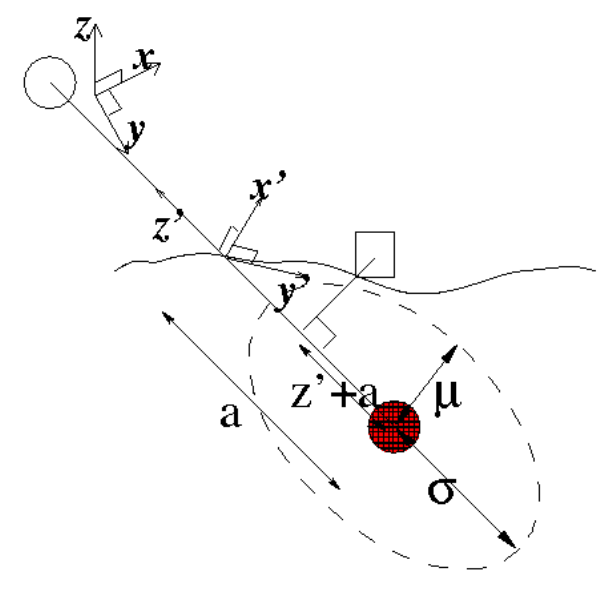

Figure 5.1: Sketch showing the reference frames, and the ellipsoid of energy distribution.

The ion source, at some random position in a plane directly above the surface plane, projects the ions along a trajectory inclined at $\theta$ to the vertical and with azimuth $\phi$. For the ion motion, periodic boundary conditions are assumed along the horizontal (i.e. parallel to the surface plane). After penetrating the solid through a depth $a$, measured along the ion trajectory, each ion comes to rest and distributes its kinetic energy $\epsilon$ within an ellipsoid with the final stopping position of the ion as centroid (see Fig. 5.1). A surface atom is eroded with a probability proportional to the energy $E(\mathbf{r})$ deposited 
there, as given by Eq. (3.6), i.e.

$$
E(\mathbf{r})=\frac{\epsilon}{(2 \pi)^{3 / 2} \sigma \mu^{2}} \exp \left(-\frac{[z+a]^{2}}{2 \sigma^{2}}-\frac{x^{2}+y^{2}}{2 \mu^{2}}\right) .
$$

We use a primed reference frame $\left(x^{\prime}, y^{\prime}, z^{\prime}\right)$, with origin at the impact point and in which the $z^{\prime}$-axis coincides with the ion trajectory, for the local

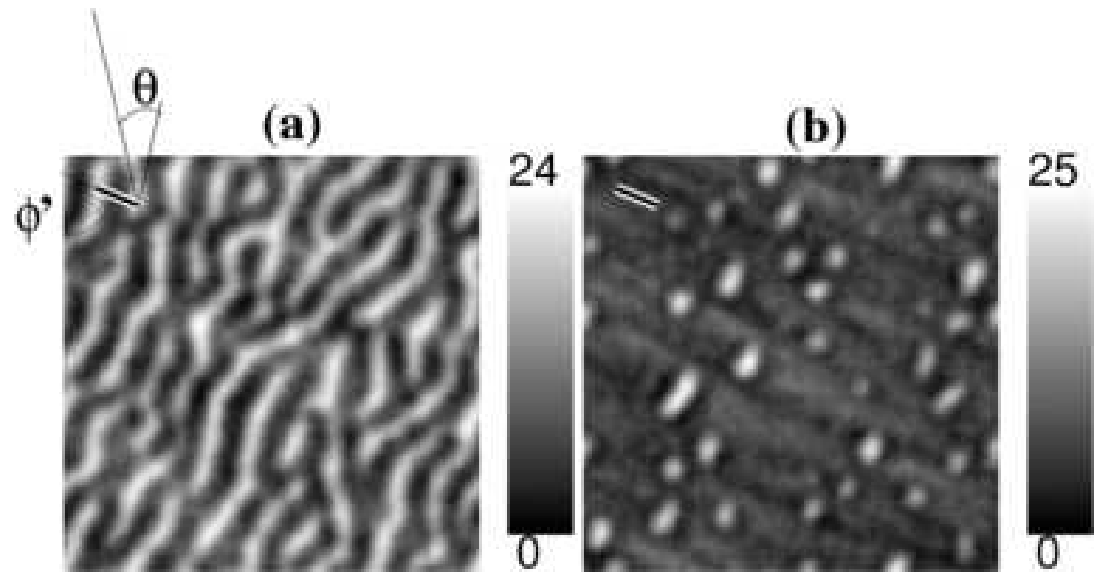

Figure 5.2: Dependence of ripple orientation on incidence angle $\theta$, and azimuth $\phi$. The bar represents a projection of the ion beam direction on to the surface plane. This projection makes angle $\phi^{\prime}=\pi / 2-\phi$ with the left boundary, as shown in (a). $\phi=22^{\circ} ; \theta=50^{\circ}$ and $80^{\circ}$ in (a) and (b) respectively. The chart gives a rough estimate of the number of monolayers eroded.

coordinates of a surface point; whereas for the ion motion, before penetration, we use the real cartesian coordinate $(x, y, z)$ system [82, 105] (see Figs. [5.1] and 5.3). All parameters depend on the experimental parameters like type of material, ion energy, and angle of incidence. For amorphous materials they can be estimated using the SRIM (the stopping and range of ions in matter) simulation package [84]. Except where otherwise stated, $a=6.0, \theta=50.0^{\circ}$, $\phi=22.0^{\circ}$, and $\epsilon$ is chosen to be $(2 \pi)^{3 / 2} \sigma \mu^{2}$. Lateral system sizes $L$ up to 512 have been studied.

We obtained ripple topographies, at off-normal incidence, whose orientation depends on both $\theta$ and $\phi$ (see Fig. 5.2). For small $\theta$ [Fig. [5.2 (a)], the ripple wave vector $\mathbf{k}$ is parallel to the projection; whereas for large $\theta$ [Fig. [5.2 (b)], $\mathbf{k}$ is perpendicular to the projection. Note that the ripples (whose troughs are the dark lines) obtained in Fig. 5.2 (b) are different from the ones obtained in 5.2 (a). In this model, it is assumed that bulk vacancies do not contribute to the surface topography, and overhangs are not allowed 
[i.e, solid-on-solid (SOS)]. Shadowing effects are included, and desorption excluded; thus enabling comparison with continuum models.

\subsubsection{Model extension: lateral erosion}

In this section, we extend the HKGK sputter-model with the inclusion of lateral erosion. This has the effect of relaxing the surface, expecially as local surface slopes increase. That is, since lower (surface) particles in a column may now be eroded due to their being closer to the stopping point of ion than topmost atom in column. As an illustration, see erosion of atoms at positions 1 and 2 in Fig. 5.3. In the previous version of the model, only the topmost surface atoms were considered for erosion; i.e, only the scenario occuring at positions 3 or 4 in Fig. 5.3, without that occuring at 1 or 2 . In the illustration (Fig. 5.3) $), d_{\|}=z+a$ is the distance of the surface atom, from the final stopping point of the ion, along the ion trajectory. And $d_{\perp}=\sqrt{{x^{\prime 2}}^{2}+y^{\prime 2}}$, is the distance of the surface atom perpendicular to the ion trajectory.

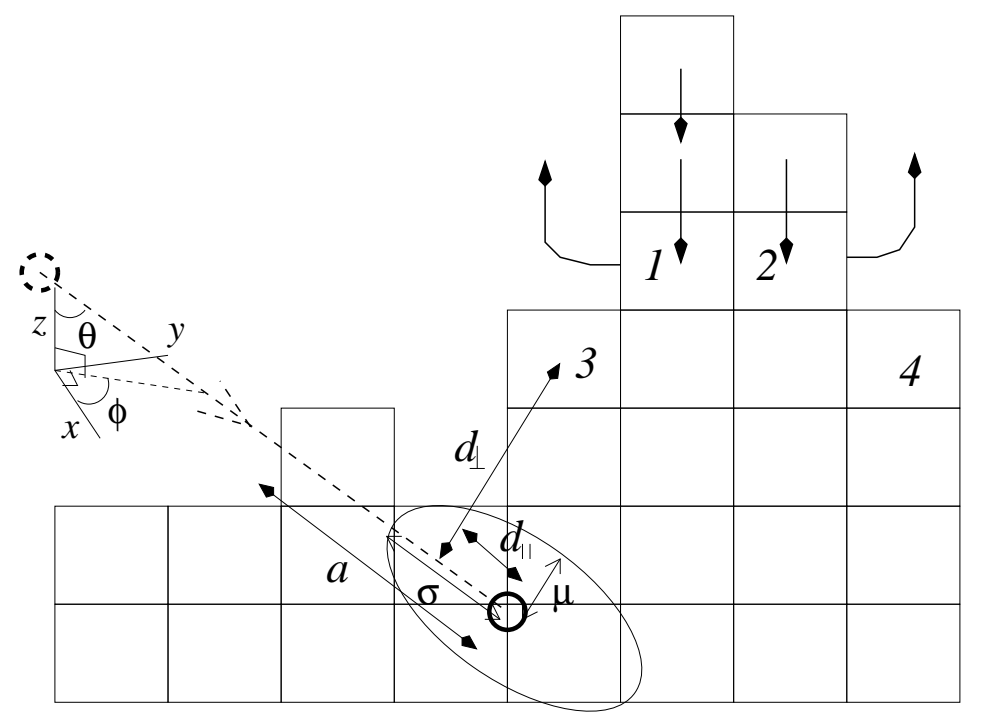

Figure 5.3: Sketch showing collision cascade ellipsoid, and relaxation of surface as a result of lateral erosion. Erosion of atom at position 1 or 2 triggers the relaxation, which ensures there is no overhang; the atom at position 3 or 4 is only eroded, without surface relaxation.

In analogy with the continuum theory, this modification (representative of non-linear effects) leads to a more rapid saturation of the surface width as shown in Fig. 5.4. As seen in the figure, the previous version (without lateral 
erosion) also stabilizes the rapid growth of the surface roughness, albeit at much longer times than in the extended version (with lateral erosion). Which shows that the previous version only has weak nonlinearities whereas the extended version accounts for stronger non-linear effects. This extension of the model is used in sections 6.2 and 6.3 .

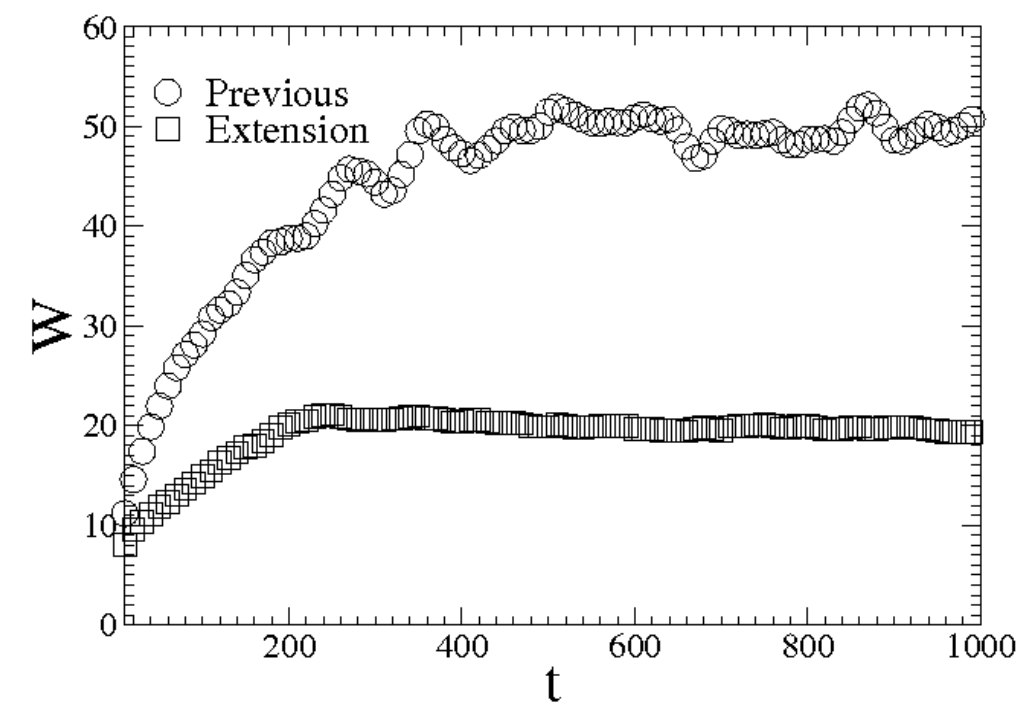

Figure 5.4: Effect of the inclusion of lateral erosion on the surface width. The circle symbols represent data (average of 50 realizations) obtained using the previous version, and the square symbols represent data obtained using the extended (i.e. including lateral erosion) version. $\sigma=3.3, \mu=1.7, a=5.4$, and $k_{B} T=0.1$ $\mathrm{eV}$.

\subsubsection{Model extension: sample rotation}

A number of experiments have reported the effects of rotation on surface morphology, with explanations from the continuum theory. As we shall see later in section 6.3. there is the need to implement Zalar rotation in the HKGK sputter-erosion model. Such Zalar rotation, at constant angular velocity $\omega$, is implemented by keeping the substrate static and then rotating the ion beam (which is equivalent to keeping the ion beam direction fixed and rotating the substrate [73]). This we simulate by choosing $\phi$ randomly such that $0 \leq \phi<2 \pi$, since, in the large $\omega$ limit, it is as if the solid were 
sputtered from all angles $\phi[50$. Surface profiles without, and with, rotation are shown in Figs. 5.5 (a) and (b), respectively.

This implementation of sample rotation is used to obtain the results presented in section 6.3.

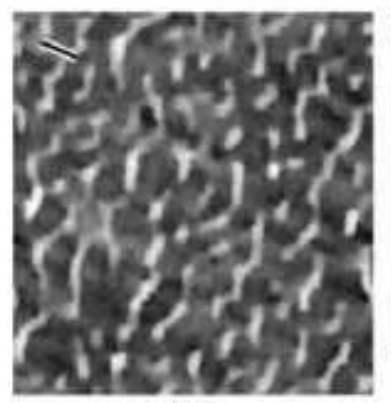

(a)

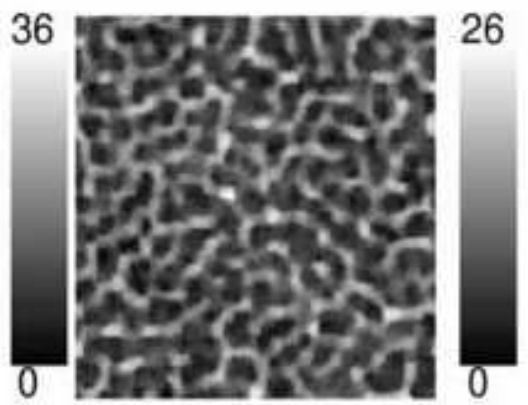

(b)

Figure 5.5: Effect of sample rotation, as implemented in the simulation. (a) Sputtering alone. (b) Simultaneous sputtering and rotation. $t=3, a=6, \theta=$ $50^{\circ}, \sigma=3, \mu=1.5, k_{B} T=0.1 \mathrm{eV}$.

\section{$5.2 \quad$ Hopping dynamics}

Surface diffusion is believed to be a thermally activated, unlimited(-mobility) process; driven only by the need to minimize the surface energy. But, in the introduction of the HKGK model to the literature of surface sputtering phenomena, the crucial role of surface diffusion mechanisms, on the resulting surface morphology, was emphasized; while using the Hamiltonian and the Wolf-Villain surface diffusion models. So, we first consider, in section 6.1. both unlimited mobility models in our study of ripple propagation and coarsening, where we found the $n=2$ Hamiltonian model to be qualitatively similar to the Arrhenius model (without Schwoebel barriers). Hence, later on (expecially in sections 6.2 and 6.3), we focus mainly on the thermally activated Arrhenius surface diffusion model. These surface migration models are fully described in sections 5.2.1 and 5.2.2. Here, we only mention the specific points as regards our work. 


\subsubsection{Hamiltonian Model}

With this model, we simulate surface diffusion precisely as described in section 5.2.1, using a temperature of $T=0.2 \mathrm{~J} / k_{B}$; i.e, using the same temperature as in Ref. [82]. A diffusion sweep is initiated after every $0.001 \mathrm{~L}^{2}$ erosion attempts. Although no exact mapping is possible we can estimate that the temperature $T=0.2 \mathrm{~J} / k_{B}$ in this model corresponds roughly to the temperature $T=0.2 \mathrm{eV} / k_{B}$ used in the second model below; i.e, it is above room temperature, and mimics thermal spikes (see below). The estimate is based on a comparison of the pure diffusion mechanism without sputtering such that they lead to comparable values of the roughness. Note that this temperature is below the roughening transition of this model [102].

Initially, for times less than about 1.4 ions/lattice site, the surface is rough and then the formation of ripples starts. In Fig. 5.6 the time development of

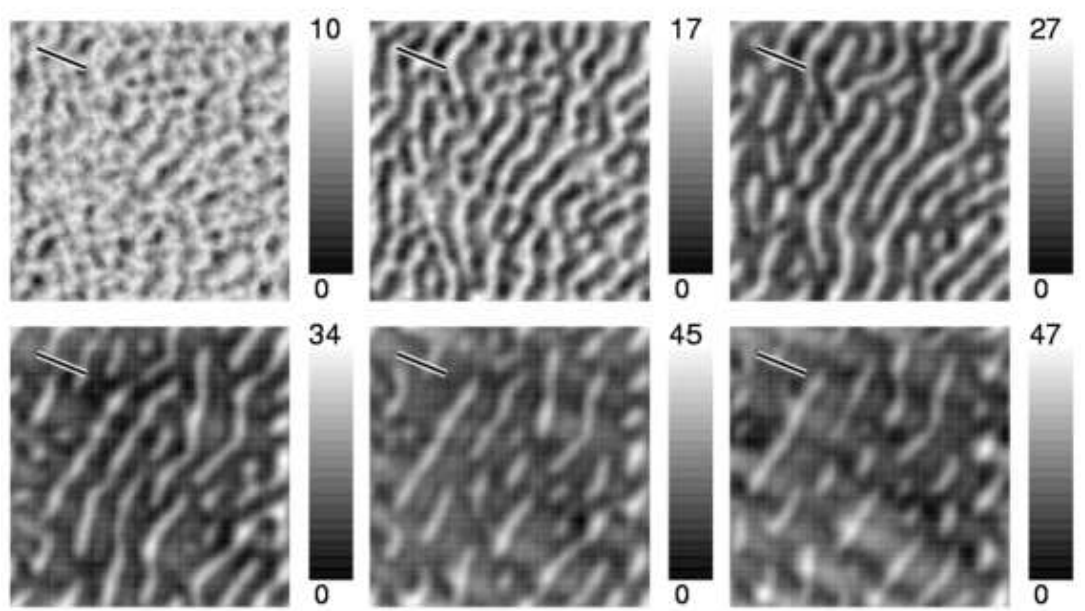

Figure 5.6: Surface profiles at a substrate temperature of $0.2 \mathrm{Jk}_{B}^{-1}$ and at different times. Starting from top-bottom, left-right, $\mathrm{t}=0.5,1.5,4.0,9.0,14.0$ and 20.0 ions/atom. Ion beam direction, indicated by the bar, is perpendicular to ripple orientation. The scales show the surface height measured from the lowest height.

a sample surface topography is shown for the Hamiltonian diffusion model. Initially ripples are formed, they propagate slowly and then disappear at longer times. We observed the same scenario for the Arrhenius model (presented below), hence, we do not include the corresponding figure for $T=0.2$ $\mathrm{eV} / k_{B}$ here. The long-time behavior, where the ripples have disappeared, 
has already been studied in Ref. [82].

\subsubsection{Arrhenius Model}

Our temperature is measured in units of $\mathrm{eV} / k_{B}$ in this model, where $T \approx$ $0.02 \mathrm{eV} / k_{B}$ corresponds to room temperature. $k_{0}=2 k_{B} T / h$ is the vibrational frequency of a surface adatom, i.e. a hopping attempt rate; $h$ is Planck's constant. The hopping attempt rate is very high, with a corresponding low hopping probability that results from Eq. (4.13); which slows down the simulation. Thus we incorporate the factor $\exp \left(-E_{S} / k_{B} T\right)$ into the rescaled attempt rate such that the hopping rate reads

$$
k(E, T)=k 1 \exp \left(-\frac{\triangle E}{k_{B} T}\right)
$$

where $k 1=k 0 \exp \left(-\frac{E_{S}}{k_{B} T}\right)$ is a much lower hopping attempt rate, $\triangle E=$ $n_{n} E_{N N}+E_{S B}$. This physical attempt rate, in comparison with the ion current density used in experiments, determines the ratio between the number of sputtering steps and the number of surface diffusion steps made in the simulation. A discussion of parameter optimization and a rescaling of the temperature with the parameters is given in Ref. [107]. Note, finally, that for atoms on top of planes, which are far from down edges, $\triangle E=0$, i.e. each hop is accepted, independently of the temperature.

In the experiments, we typically have $N=1 \times 10^{15}$ atoms $/ \mathrm{cm}^{2}$ on the surface. Since the typical experimental ion current density is of the order $F=7.5 \times 10^{14}$ ions $/\left(\mathrm{cm}^{2} \mathrm{sec}\right)$, this implies a flux of $\phi=F / N \approx 0.75$ ion atom $^{-1} \mathrm{sec}^{-1}$. From the values given above, we get effective hopping attempt rates $k_{1}$ of around $2001 / \mathrm{s}$ for room temperature, hence 200 sweeps of the diffusion mechanism correspond to 0.75 ions per surface atom. Thus, with this model, we initiate a diffusion sweep every $\phi L^{2} / k 1=0.0037 \mathrm{~L}^{2}$ erosion steps.

Here, one can choose a temperature corresponding to the physical temperature present in the experimental system. A naive guess is to use room temperature $k_{B} T=0.02 \mathrm{eV}$, at which the experiments usually are carried through. The resulting structures are shown in Fig. 5.7, for intermediate as well after long sputtering times. We cannot observe clean ripples. The reason is that this kind of diffusion mechanism is too slow at room temperature to effectively counteract the strong roughening due to our model of sputtering, which possesses a particularly high sputtering yield. Hops are almost always prevented if an atom has in-plane neighbors, so the mechanism is not very effective on a rough surface. Since the surface relaxation is essential for the 


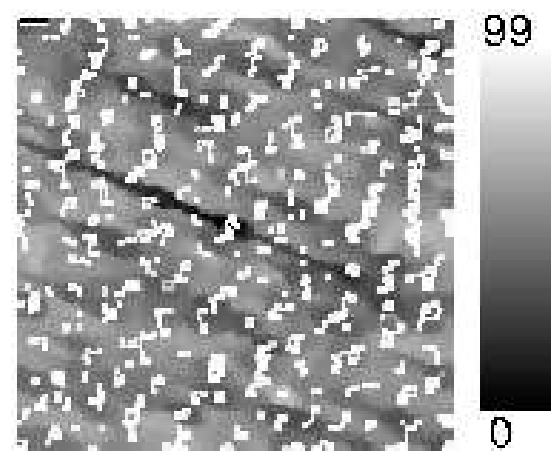

Figure 5.7: Sample surface topography for a small system $(\mathrm{L}=128)$, for the Arrhenius $\mathrm{MC}$ diffusion mechanism at surface temperature equal to room temperature, after $t=100$ ions/atom. No clear ripples can be observed. Similar results were observed for almost all time, except the very early ones.

formation of ripples [4], it needs locally higher than room temperatures to produce clean ripples in our model. This happens indeed in experiments, since most of the kinetic energy, carried by the incoming ion, is converted into lattice vibrations, hence the surface is locally strongly heated. Here, we do not know the spatio-temporal distribution of the local temperature. Either one would have to perform MD simulations, or include heat conduction in the model, both making the treatment of large systems over long time scales infeasible. Instead, we are choosing a higher but constant effective temperature $T$, which is a good first approximation.

Now, we want to estimate this effective temperature. The most basic approach is to describe the energy carried by the ions as a constant inflow of energy at the surface, fix the temperature far away from the surface to room temperature and solve the stationary heat-conduction equation to calculate the temperature at the surface 108. The resulting temperature depends strongly on the ion energy, the ion current density, and the thermal conductivity of the material. For experimentally reported parameters, temperature rises up to $1500 \mathrm{~K}\left(0.155 \mathrm{eVk}_{B}^{-1}\right)$ are found [108. This shows that high efficient temperatures, even in the stationary state, may be achieved. However, in the experiments of Habenicht et al. [22] only small average ion current densities have been used, which result in a temperature rise at the surface of only few K.

This does not mean that one can use a temperature close to room temperature as effective temperature. The reason is that right after impact, the surface is strongly heated close to the melting temperature and the quickly cooled again, i.e. a thermal spike occurs [109]. Furthermore, the surface is 
sputtered using a focused ion beam (of diameter $30 \mathrm{~nm}$ ), which is moved relatively slowly over the surface and which exhibits a large spot current of $15 \mu \mathrm{A} / \mathrm{cm}^{2}$. Hence, under the ion beam, for several short time intervals, surface diffusion is greatly enhanced. Marks has calculated [110] the spatiotemporal development of the temperature after ion impact by solving the dynamic heat-conduction equation, resulting in a temperature profile $\tilde{T}(r, t)$ as function of time $t$ and distance $r$ from the point of impact. The initial distribution $\tilde{T}(r, 0)$ is given by a step function with $\tilde{T}(r, 0)$ being the melting temperature of the material for $r \leq r_{0}$ and being the room temperature elsewhere. The initial radius $r_{0}$ is determined such that the thermal energy inside this semi sphere equals to the energy carried by the ion. Marks found that the surface is heated strongly right after the impact and is cooled down to temperatures close to room temperature within few ps. Qualitatively and

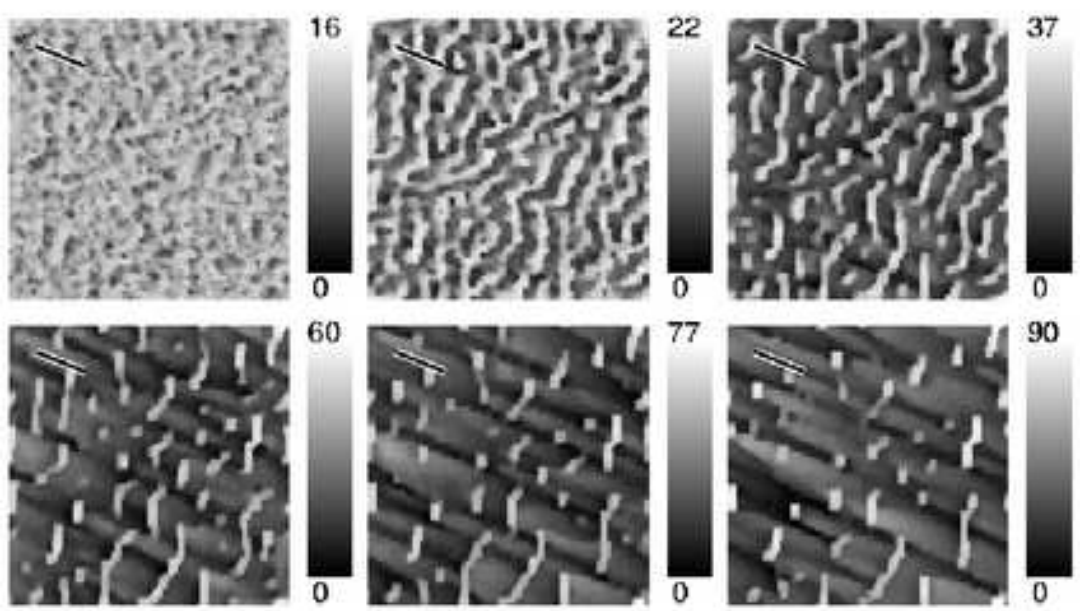

Figure 5.8: Surface profiles at a substrate temperature of $0.1 \mathrm{eV} / k_{B}$ with the Arrhenius diffusion model. Starting from top-bottom, left-right, $\mathrm{t}=0.5,1.5,4.0$, 9.0, 14.0 and 20.0 ions/atom. In both cases, depicted here and in Fig. 1, ripples propagate along a direction opposite to that of the ion beam.

quantitatively similar profiles have been observed in MD simulations [11] as well. We apply his equation, using the parameters for ion energy and ion current density in the spot as given above, to determine an effective temperature (with $r_{0}=1.56 \mathrm{~nm}$ in our case). The basic idea is that in a time interval $\Delta t$, the number of hops governed by the temperature $\tilde{T}(0, t)$ at the 
impact point should be the same as under the effective temperature $T$ :

$$
\int_{0}^{\Delta t} k_{0} \exp \left(-\frac{\Delta E}{k_{B} \tilde{T}(0, t)}\right) d t=\Delta t k_{0} \exp \left(-\frac{\Delta E}{k_{B} T}\right)
$$

We have neglected here the temperature dependence of $k_{0}$. When including it, we found that the resulting effective temperature changes only slightly. We have chosen $\Delta t$, as the average time between two ions arriving in a circle with area $\pi r_{0}^{2}$ under the ion beam spot, resulting in $\Delta t=1.4 \times 10^{5} \mathrm{ps}$. For the energy barrier, we have chosen $\Delta E=E_{S B}+3 E_{N N}+E_{S}$, which corresponds to atoms along edges of islands/steps. Using these parameters, we found an effective surface temperature of $T=1200 \mathrm{~K}$, i.e. considerably higher than room temperature. In this calculation it is assumed that only the energy carried by the ions hitting the "target area" $\pi r_{0}^{2}$ contribute to the heating of the surface inside the area. If one takes into account that also the ions hitting the neighborhood of the target area contribute to the heating inside the area, even higher effective temperatures can expected.

The exact effective temperature depends on many parameters as ion energy, ion current density, heat conduction, surface roughness etc. We are here interested only in universal effects, not in modelling a specific experimental setup. For this reason, we use the above result only as a guideline and study several temperatures of, and above, this order of magnitude. Hence, for the further analysis of ripple movement, we consider high effective temperatures for the Arrhenius MC model, such that the surface diffusion is indeed able to act as an effective smoothing mechanism (see Fig. 5.8). At such higher temperatures we observe some universal features for both diffusion mechanisms, as presented in section 6.2 .

For surface diffusion we start to concentrate on the Arrhenius diffusion mechanism from section 6.2. for the rest of this work. We use an effective temperature of $k_{B} T=0.1 \mathrm{eV}$. 
This most beautiful system

[The Universe] could only

proceed from the dominion

of an intelligent and power-

ful being

Sir Isaac Newton

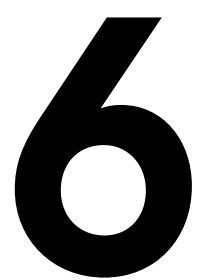

\section{Results and Discussion}

In this chapter we shall present and discuss the results of our study [105, 106] of self-organized pattern formation on, and the morphology of, ion sputtered surfaces by means of Monte Carlo simulations. The sputtering and surface diffusion models, as well as the simulation parameters, used in this study have been discussed in chapter 5 .

Motivated by the experiment of Ref. 22, we first study ripple propagation and coarsening. In this experiment, the observed velocity dispersion of propagating ripples raised the question of whether, or not, it was due to a crossover to the non-linear regime. In the KPZ model, non-linearities are shown to arise from the attachment of newly deposited particles, to the sides of protrusions on growing surfaces; this is analogous to lateral erosion of particles. Indeed, it was demonstrated in section 3.3 (see Fig. 3.2), that lateral erosion of a surface protrusion occurs if the nonlinear coefficient is negative. But in the earlier version of the HKGK model [82, no such lateral erosion of particles was considered. Thus, one may expect weak nonlinearities in the earlier version, which implies that it should not be able to reproduce the velocity dispersion; if strong or increasing non-linearities are responsible for it. Hence, in the next section, we have used the earlier version of the HKGK model, in which the erosion of only the topmost surface atoms are considered.

Using an extension of the HKGK model that includes lateral erosion (see section 5.1.1), we explore the full topographic features of the model in section 6.2, vis-a-vis the effects of collision cascade parameters on the surface morphology. By so doing, in a certain parameter regime, we obtained oblique incidence dot formation, without sample rotation. Since dots have previously been found to arise from simultaneously rotated and sputtered surfaces, this raises the question of the role of concurrent rotation as the surface is sputtered. Does rotation widen the sputtering conditions under which dots are 
formed? Does it create different and/or "better" dots? Or, since sample rotation has been shown, in experiments and theory, to considerably smoothen surfaces that are simultaneously sputtered, does it then reduce the height and/or width of the dots?

In order to answer these questions we used another extension of the HKGK model, described in section 5.1.2, in which sample rotation was incorporated (according to a hint given in the theory in section 3.7.2). The results of such simulation of simultaneous sputtering and rotation are presented in section 6.3, which rounds up the chapter.

\subsection{Ripple Coarsening and Propagation}

In the experiments of Ref. 22 the ripples generated during gallium ion beam erosion of silicon were observed to propagate with a velocity that scales with the ripple wavelength as $v \sim \lambda^{k}$, where $k \approx 0$ initially, and $k=-1.5$ after a crossover wavelength $\lambda_{c} \approx 100 \mathrm{~nm}$. In this section we focus on intermediate times, where the transition, observed in the experiments, may likely occur. Our results corroborate the experimental observation, but in addition, we find that while the ripple coarsening $[\lambda(\mathrm{t})]$ follow a power law at low temperatures, the trend is exponential at high temperatures; where the ripples first come to rest before vanishing.

Some profiles of the time evolution of the surface topography have already been shown in sections 5.2.1 and 5.2.2 (Figs. 5.6 and 5.8, respectively). In this section we first explain how we study the movement of the ripples. Then, we present and discuss our simulation results.

\subsubsection{Ripple Kinematics}

In order to monitor the ripple propagation on the computer, we assign the crest points of the ripples to clusters (see Fig. 6.1), and then monitor the motion of these clusters. A cluster of crest points is defined as the set of surface points with height $h(x, y, t) \geq h_{c}$ and nearest neighbor distance $l \leq l_{c}$, where $h_{c}$ and $l_{c}$ are cut-off surface height and distance between neighboring cluster points respectively. We have chosen our cut-off height to be a function of the average height $\langle h\rangle$ of the configuration, and the height difference $d_{h}$ between the maxima and minima of the surface; i.e, $h_{c}=\langle h\rangle+p d_{h}$, where $p$ is some fixed percentage. In this way clusters with about the same proportionate sizes can be followed from the beginning of ripple formation until complete disappearance of the ripples. Furthermore, we have used $l_{c}=2$. Different, unconnected ripples should, in general, generate different clusters. We also 
require that the number $N$ of elements in a cluster be large enough to allow for statistical analysis, here we have chosen $N \geq 10$ elements.
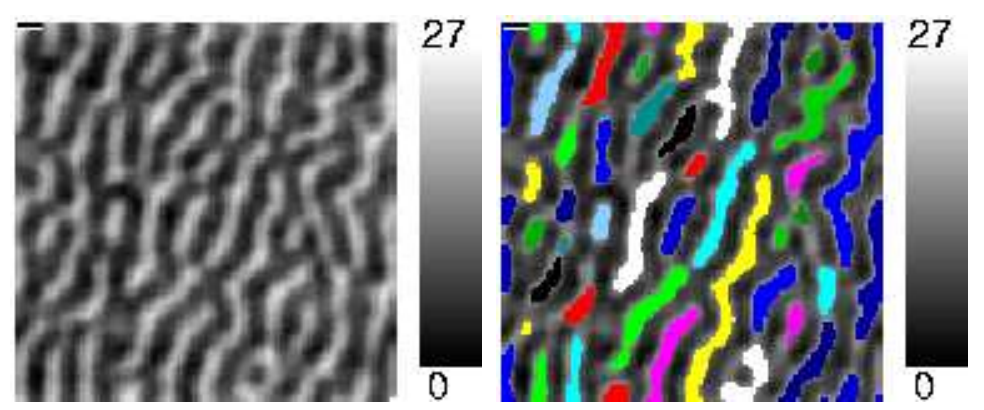

Figure 6.1: Surface profile for time 3 ions/atom (thermodynamic diffusion model, $T=0.2 \mathrm{~J} / k_{B}, L=128$ ). In the second figure (on the right) the clusters formed from the profile on the first figure are printed on top of the profile.

The propagation of the ripples is studied by calculating the time rate of change of the position of the centre of mass of a cluster

$$
\dot{\mathbf{x}}_{C M}=\frac{\sum_{i} m_{i} \dot{\mathbf{x}}_{i}}{\sum_{i} m_{i}},
$$

where the summation is over all the elements of the cluster. We have assumed a homogeneous system composed of unit mass particles, such that the center of mass of a cluster is $\mathbf{x}_{C M}=N^{-1} \sum_{i} \mathbf{x}_{i}$. The ripple wavelength is given by $\lambda=2 \pi / \eta, \eta$ being the average expectation value of the Gaussian fitted to the peak of the structure factor $S(\mathbf{k}, t)=|h(\mathbf{k}, t)|^{2}$. Where $h(\mathbf{k}, t)$, the fourier transform of the surface height $h(\mathbf{r}, t)$ at time $t$, with mean $\langle h\rangle$, is given by

$$
h(\mathbf{k}, t)=L^{-d / 2} \sum_{\mathbf{r}}[h(\mathbf{r}, t)-\langle h\rangle] e^{i \mathbf{k} \cdot \mathbf{r}} .
$$

Fig. 6.1 shows one profile of the surface for system size $128 \times 128$ at time $\mathrm{t}=3$ ions/atom; in the second figure, we print the clusters on top of their corresponding ripples. As seen in the figure of the clusters, application of periodic boundary conditions neccessitates the need to first unfold toroidal clusters before calculating the position of their center of mass. As time increases, local surface slopes $\nabla h$ increase, and since the non-linear effects depend on the square of $\nabla h$ they will dominate by scaling down surface relaxation mechanisms [1. These non-linear effects are responsible for the disappearance of ripples (Fig. [5.6) at long times, and for the transition of the surface topography from a periodic ripple pattern to a rough topography 
with self-affine scaling [39, 4]. We thus expect fluctuations in the position of the centre of mass due to disappearing ripples; the fluctuations are averaged out by using systems of size $512 \times 512$ with a large number of clusters such that the ripple velocity at any time is an average of the velocities of all the ripples at this time.

\subsubsection{Results}

The results are obtained, as already mentioned, for square lattices of size $512 \times 512$, with periodic boundary conditions, and as an average over fifty different realizations.

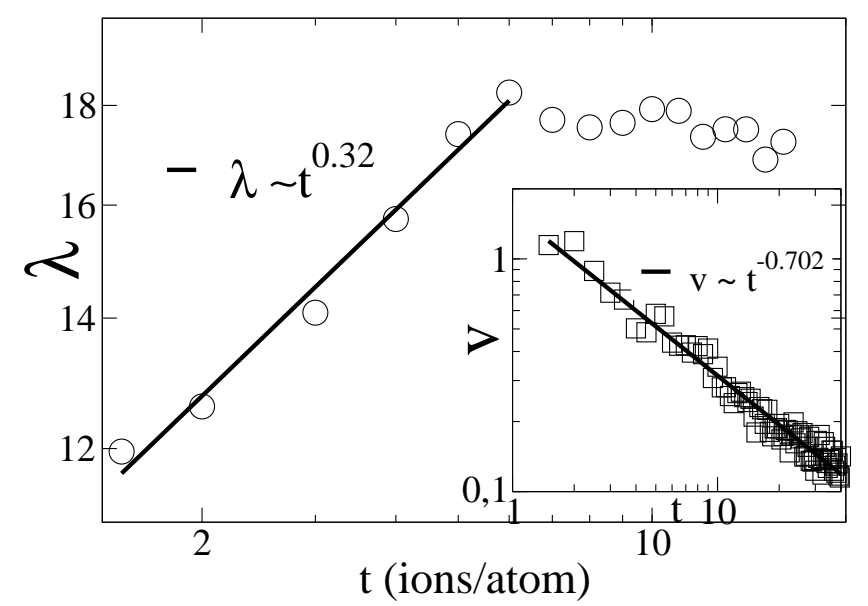

Figure 6.2: Ripple wavelength, $\lambda$, measured in lattice units, as a function of time, $t$. The inset shows the time dependence of the ripple propagation velocity, $v$ (measured in lattice units per ion per atom). Both results are for the kinetic diffusion mechanism, at a substrate temperature of $k_{B} T=0.1 \mathrm{eV}$.

Figure 6.2 is the plot of the ripple wavelength (circle symbols) versus time measured in units of the number of ions per atom; its inset is a plot of the projection of the ripple velocity along the ion beam direction, versus time, both at the estimated effective temperature of $k_{B} \mathrm{~T}=0.1 \mathrm{eV}$ corresponding to the experimental conditions from Ref. [22].

A plot of wavelength versus time in Fig. 6.2 reveals that for short times $\lambda \sim t^{\varepsilon}$, where $\varepsilon=0.32$, consistent with experimental results 0.5 and 0.26 of Ref. [22] and Refs. [38, 30]. But we observed a power-law behavior only in 
the initial stages of ripple formation, the wavelength becoming constant in time at the later stage.

The velocity shows a power-law behavior over a larger time interval, resulting in $v \sim t^{-0.7}$ as obtained from inset of Fig. 6.2. This is in excellent agreement with the experimental result $v \sim t^{-0.75}$ of Habenicht et al. [22]. A difference is that for smaller times a constant velocity was observed in the experiments, while we do not see any clean ripples for smaller times than the power-law regime. Combining both scaling results gives $v \sim \lambda^{-2.19}$, in good

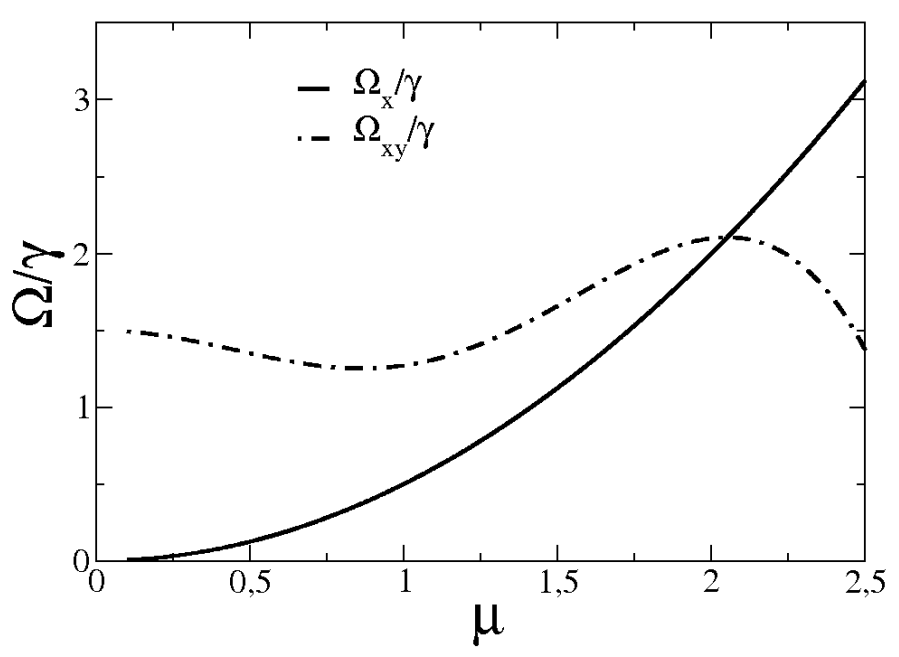

Figure 6.3: Plot of the ratios $\Omega_{x} / \gamma$ and $\Omega_{x y} / \gamma$, of the linear coefficients appearing in Eq. (3.17). $\sigma=3.0, \mu=1-2.5$.

agreement with the exponent -2 of continuum theory [39] [see Eq. (3.17) in section 3.4.2, provided that the linear coefficients $\Omega_{x}$ and $\Omega_{x y}$ are not negligible in this anisotropic collision cascade case. A calculation of the higher-order linear coefficients $\Omega_{x}$ and $\Omega_{x y}$ for the anisotropic collision cascade cases $\sigma=$ 3.0 and $\mu$, ranging from 1 to 2.5, indeed reveals their significance, relative to the first-order linear coefficient $\gamma$, for our simulation parameters (see Fig. 6.3). Thus, our result for the velocity dispersion is in agreement with both the experiment and the continuum theory. Which implies that higher-order linearities are responsible for the observed dispersion.

Now we turn to higher effective subtrate temperatures, corresponding e.g. to higher ion currents and/or materials with lower heat-conductivity. Figures 6.4 and 6.5 are plots of the ripple wavelength (circle symbols) as a function of time, at respective temperatures $k_{B} T=0.2 \mathrm{~J}$ and $k_{B} T=0.2 \mathrm{eV}$; 


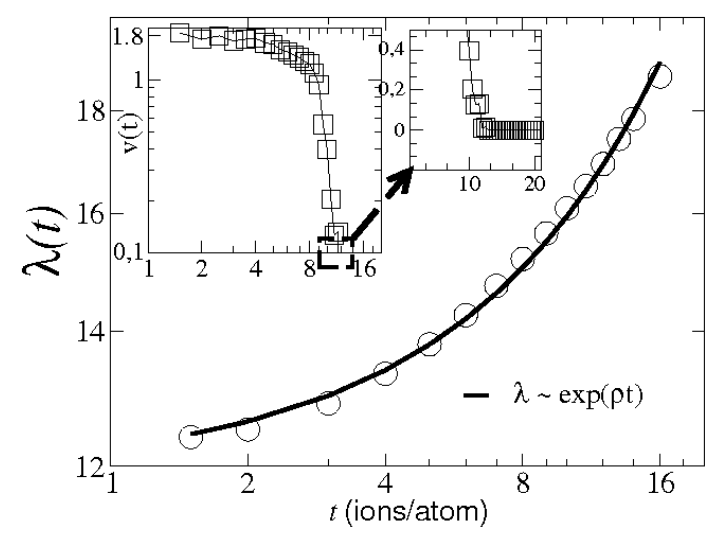

Figure 6.4: Ripple wavelength, $\lambda$, measured in lattice units, as a function of time, $t$. The main inset shows the time dependence of the ripple propagation velocity, $v$ (measured in lattice units per ion per atom); the smaller inset is a magnified view of $v(t) \approx 0$ in the main inset. The bold line in the main plot is a fit of the simulation data to an exponential function. The line drawn in the insets are to guide the eyes. Both results are for the thermodynamic diffusion mechanism, at a substrate temperature of $0.2 J k_{B}^{-1}$.

using the first and second models of surface diffusion respectively. In both models, the ripples disappear after a while, i.e. the ripple wavelength diverges. Considering the lifetime of the ripples from first appearance to annihilation, the wavelength increases exponentially with time as $\lambda \sim \exp (\rho t), \rho=0.029$ (Fig. 6.4 ), in the first model, while it increases with time according to the inverse law $\lambda(t) \sim 1 /\left(c_{1}-c_{2} t\right)$ with $c_{1}=0.083$ and $c 2=0.0036$ (Fig. 6.5) in the second model. To investigate the origin of the difference, we performed also simulations with the Arrhenius model, but with the Schwoebel term set to zero. In this case the result was very similar to result in Fig. 6.4 of the thermodynamic model (which has no Schwoebel term here), and we obtained a behavior $\lambda \sim \exp (0.036 t)$. On the other hand, when we set the energy in the Schwoebel term to twice its value, $E_{S B}=0.3 \mathrm{eV}$, the result is very similar to $E_{S B}=0.15 \mathrm{eV}$. This shows that the Schwoebel barrier plays an important role in the pattern formation process.

The insets of Figs. 6.4 and 6.5 are plots of the ripple velocity (line with square symbols) as a function of time. Irrespective of which surface diffusion mechanism is employed, the velocity is at first almost independent of time, then it disperses after a transition time $t_{r}$. This initial plateau is similar to the plateau observed in the experiments, but the drop in velocity is very 


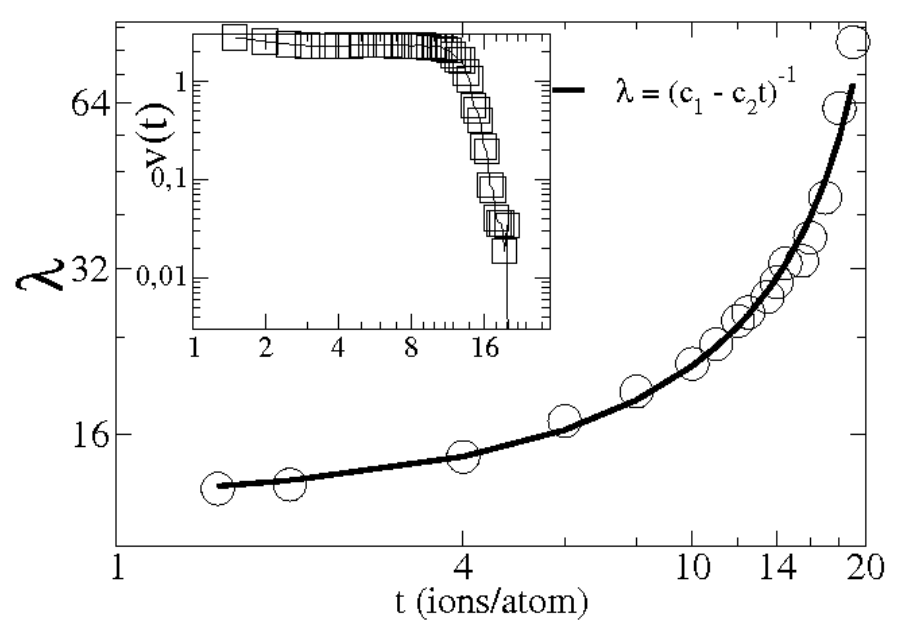

Figure 6.5: Same plot as in Fig. 6.4 but for the Arrhenius diffusion mechanism, for a substrate temperature of $0.2 \mathrm{eV} / k_{B}$. In both figures, the line with circle symbols represent the wavelength while the line with square symbols represent the velocity. In the main plot the bold line is a fit of the data to an inverse law.

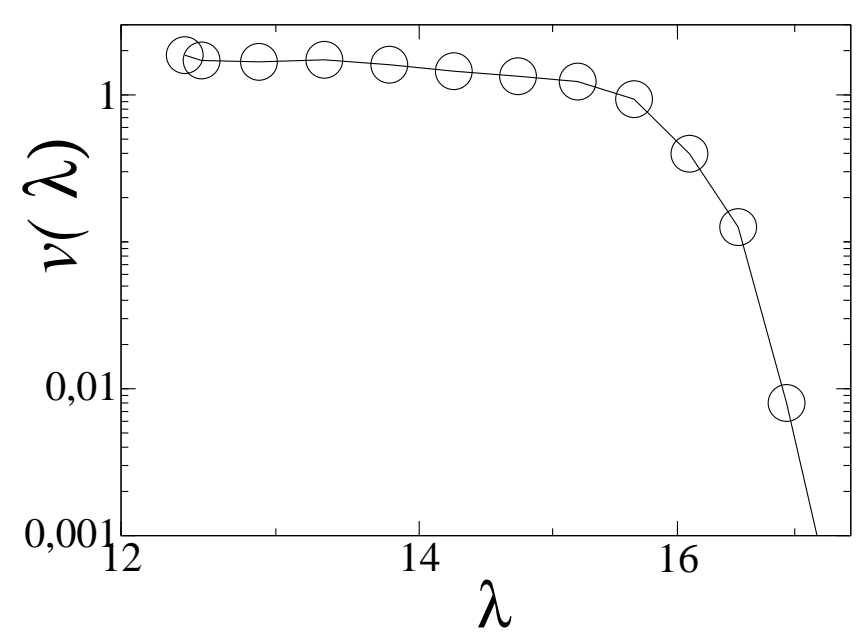

Figure 6.6: Ripple velocity as a function of ripple wavelength, for the thermodynamic and, in the inset, for the Arrhenius surface diffusion mechanism. 
abrupt, no clear power law is visible then. Moreover, the ripples finally come to rest before completely disappearing, as seen in the smaller inset of Fig. 6.4. We find, however, that at the lower temperature in the kinetic model, the ripples do not stop moving until their disappearance. Figure 6.6] shows the dependence of the ripple velocity on the wavelength for $k_{B} T=0.2 \mathrm{eV}$ resp $0.2 J$, their order of magnitude relationship is about the same as in the experiment. We see in Fig. 6.7 that the trend in velocity variation is the same at high temperatures but the magnitude increases with temperature, as one would expect from the temperature dependence of the surface diffusion. But we only observed a power-law scaling at temperatures below $k_{B} T \approx 0.18 \mathrm{eV}$. This indicates that the presence of power-law scaling of ripple wavelength and velocity, and the corresponding exponents, depend on the time scale of observation (Fig. 6.2), as well as on the effective temperature.

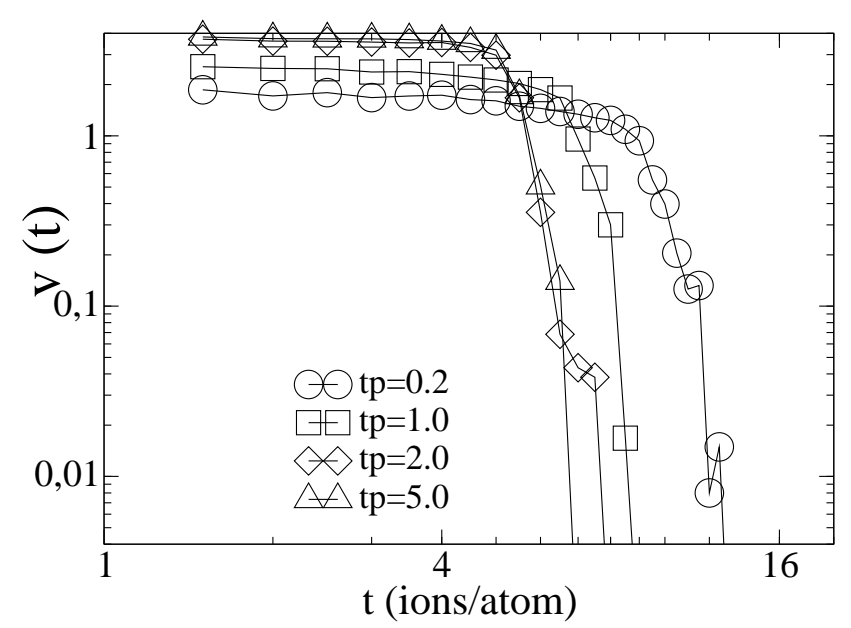

Figure 6.7: Temperature dependence of the ripple velocity; for the Hamiltonian model. Temperature is in units of $J k_{B}^{-1}$.

It seems that the increase in magnitude of the velocity, when measured at same time $\left(t<t_{r}\right)$ but different temperatures, does not continue indefinitely in our model. In Fig. 6.7 there is very little difference in the magnitudes of the velocity at temperatures 2.0 and $5.0 \mathrm{Jk}_{B}^{-1}$; even though the temperature difference is very high. This saturation behaviour is also displayed in the ripple wavelength at the same higher temperatures, as seen in Fig. 6.8. In principle, one can still fit an exponential law to the data, except that the decay constant $\rho$ in the exponential becomes very small (It has the respec- 
tive values of $0.029,0.018,0.0031$, and 0.003 from the lowest to the highest temperature.).

So for very high effective temperatures we could equally well fit a powerlaw. Hence, there may be some "critical substrate temperature", above which the wavelength remains nearly constant in time; and the velocity, after some time $t_{r}$, drops instantaneously to zero. Nevertheless, the temperature where such a "transition" will take place, is probably unphysically high (see below), so that the material used in the experiment would start to evaporate before reaching this point. But other materials, in combination with high ion currents, might quite display such a behavior. Up to the publication of this work [105], we were only aware of one set of room-temperature experiments [22]. But recent experiments [85, 113] on sputtered silicon surfaces at elevated temperatures $\left(500^{\circ} \mathrm{C}\right.$ and above) have confirmed our results of the exponential increase of the wavelength (or exponential decrease of $|\mathbf{k}|$ ) with time at higher temperatures. It would still be very interesting to see, whether the disappearance of the coarsening can be seen in experiments at higher effective temperatures corresponding to high ion currents and/or higher lab temperatures.

Our results for the second diffusion model also indicate that in $\lambda(t) \approx$ $1 /\left(c_{1}-c_{2} t\right), c_{2}$ approaches zero with increasing temperature. Here, where we can measure the temperature in real units, it is clear that the "transition" to almost non-coarsening ripples, takes place at unrealistically high temperatures $2-5 \mathrm{eV} / k_{B}$, where the material starts to evaporate. To summarize, ripple propagation depends on the effective substrate temperature as well as diffusion mechanism. At around so-far experimentally realized temperatures, ripples propagate, from first appearance, with decreasing velocity until disappearance without full cessation of motion. At high effective temperatures, however, immediately after ripple formation, the ripples move with constant velocity for some time, after which they begin to deccelerate (insets of Figs. 6.4, 6.5) and after some time, depending on the diffusion model, the ripples stop moving but keep disappearing gradually. At the same time the ripple structure is gradually being washed out, and in the final stage the ripples are completely wiped out. The ripple wavelength is always increasing in time at high temperatures, while at low effective temperatures it initially increases with time, and later becomes constant. At long times the ripples vanish [82].

\subsection{Morphological Regions}

According to the continuum theory (see sections 3.4.2 and 3.5.2), ripples arise, for all $\theta$, from the curvature dependence of the sputter yield. In the 


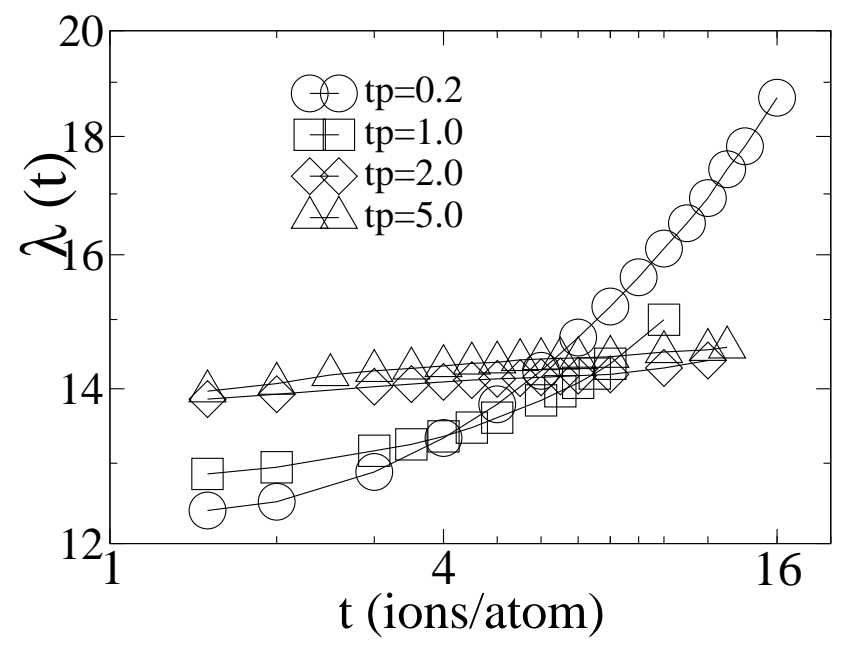

Figure 6.8: Temperature dependence of the ripple wavelength; for the Hamiltonian model.

absence of non-linearities, the $\theta$ dependent negative surface tension coefficients $\nu$, being different along the parallel and perpendicular directions to ion projection, govern the ripple orientation. However, contrary to the predictions of the continuum theory (see Figs. 3.3 and 3.4), no ripples were observed in Ref. 14] for $\theta \lesssim 40^{\circ}$ under Xe ion irradiation of Si.

In this section, we dicuss possible topographies, relevant to experimental sputtering conditions, that are obtainable from this model. We show that the angle $\theta_{r}$ around which ripple formation occurs depends on the longitudinal straggle $\sigma$ of the ion beam, with higher $\sigma$ resulting in lower $\theta_{r}$; and our results indicate the crucial role of $\mu$ to ripple formation. We found a transition from hole topography at an early time, to ripple topography at a later time, for the same cascade parameters. Also, in this section, we show dots/nanosized islands similar to those observed in Refs. [25, 30], and predicted in Refs. [70. 74] without sample rotation; arising from a transition from the ripple phase, to the QD phase, as the cascade parameters are varied beyond the isotropic case of the continuum theory.

The rest of the section is as follows: first we present the profiles obtained from our simulation, using the HKGK model with lateral erosion (as discussed in section 5.1.1). These profiles show the possible topographies for collision cascade parameter combinations accessible to experiments. This will be followed by an analysis of the $2 \mathrm{D}$ power spectral density, which allows us 
to determine quantitatively an upper bound for clear ripple formation, and other results of the cascade parameters for which oblique incidence dot formation is possible, without sample rotation.

\subsubsection{Possible Topographies}

In this section we obtain the possible topographies relevant to the experiments, by systematically varying the values of $\sigma$ and $\mu$, mimicing different material combinations; in contrast with previous studies where only single values of these parameters were used. This leads to different surface topographies, as shown below.

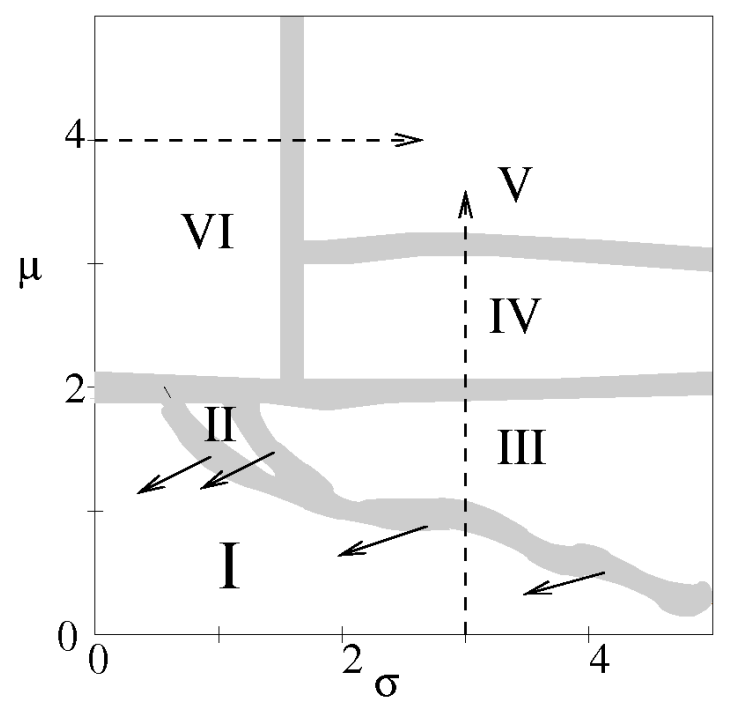

Figure 6.9: Different topographic regions $\left(\theta=50^{\circ}, a=6\right)$. Region I: rough surface; II: holes; III: clear ripples oriented perpendicular to ion beam direction; IV: short ripples (resulting from increased $\mu$ ); V: dots; VI: non-oriented structures. The short arrows indicate the evolution of the boundaries between different regions with respect to time. Hence, region III grows at the expense of region I, while region II describes only a short transient. The long dashed arrows indicate the directions referred to in Fig. 6.15.

We have studied about fifty different $(\sigma, \mu)$ combinations. We observed six different types of the qualitative behavior, corresponding to six different regions in the $(\sigma, \mu)$ space. In Fig. 6.9, these six topographic regions are indicated for $t=3$ ions/surface atom, at which almost all the surface to- 
pographic features are distinct; the corresponding profiles are shown in Fig. 6.10. The boundaries shown in this sketch do not represent sharp transitions from one topography to another, as we shall see below (in Figs. 6.17 and 6.18). Rather we observe often a smooth crossover from one behavior to the other. For this reason, we have represented them in the manner shown in Fig. 6.9. Also, we have focussed on a typical time, corresponding to time scales often used in experiments, which exhibit a rich behavior as a function of the straggling parameters $\mu$ and $\sigma$. Only one systematic change of the boundaries occurs with time, which is described below. Finally, although the sketch is specifically for $\theta=50^{\circ}$, similar "phases" also occur at other values of $\theta$, with slight deviations at the boundaries. We performed SRIM simulations 84 to estimate the following experimental parameters, that might yield parameter combinations shown in the figure. Please note that the following list is just to give a few examples. Region V: $1.5-1.7 \mathrm{keV}$ Ne-ion sputtering of $\mathrm{Cu} ; 1.2-1.4 \mathrm{keV}$ Ne-ion sputtering of Ge. Region IV: 650 - 800 eV Ne-ion sputtering of Si. Region III: $800 \mathrm{eV}$ - $1.1 \mathrm{keV}$ Ar-ion sputtering of Si; $550-700 \mathrm{eV}$ Ne-ion sputtering of C. With fluences of the order of $10^{14}$ - $10^{15}$ ions/cm2. Note that for most materials and parameter combinations $\sigma \leq \mu$, hence the region VI might be difficult to access.

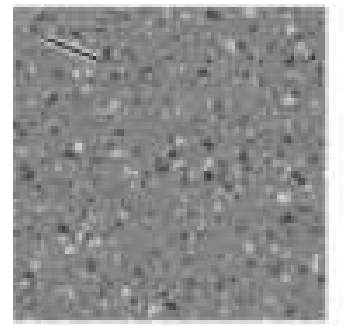

(a)

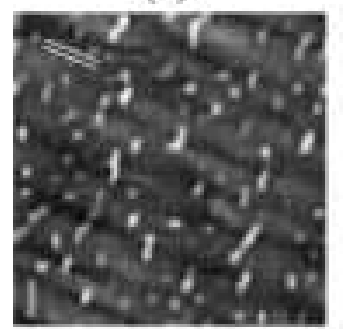

(d)
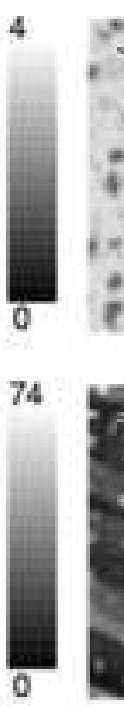

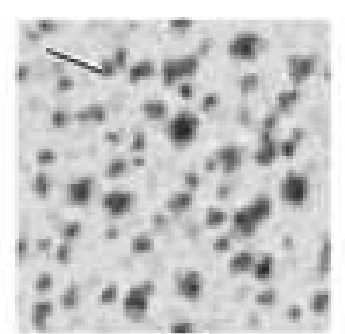

(b)

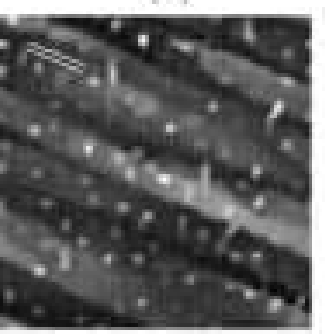

(e)
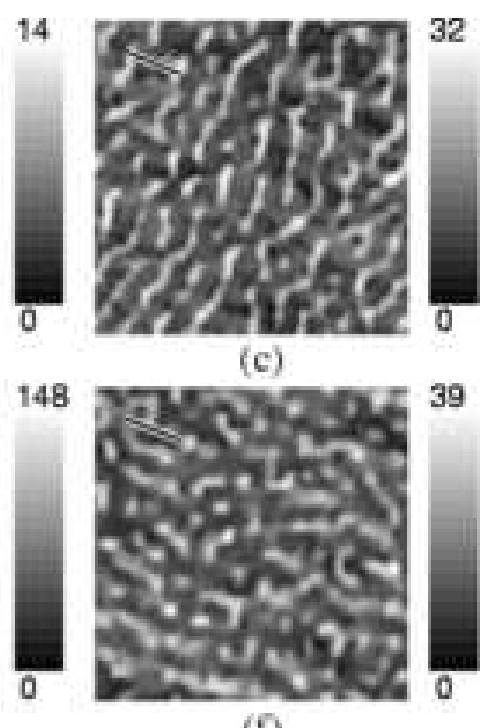

(f)

Figure 6.10: Profiles for parameters chosen from each topographic region in Fig. 6.9] $\theta=50^{\circ}, a=6, t=3.0$. I: $\sigma=1, \mu=0.5$; II: $\sigma=1, \mu=1.5$; III: $\sigma=3$, $\mu=1.5$; IV: $\sigma=4, \mu=2.5$; V: $\sigma=5, \mu=5$; VI: $\sigma=0.5, \mu=5$. The bar denotes the ion beam direction. 
Also, SRIM simulations reveal that very large $\sigma$ and $\mu$, i.e, beyond the values considered here, are impractical, since they can only occur for a higher $a$. Whereas, the value of $a$ is itself restricted by the range of ion energies that lead to ripple formation. We are not aware of an experimental study of the sputtering behavior where the parameters are varied systematically in the $\sigma$ - $\mu$ plane. Hence, when using these parameters in experiments, one might be able to observe new surface topographies.

A brief description of each topographic region in Fig. 6.10, including the behavior at later times $t \geq 3$ (ions/surface atom), is as follows:

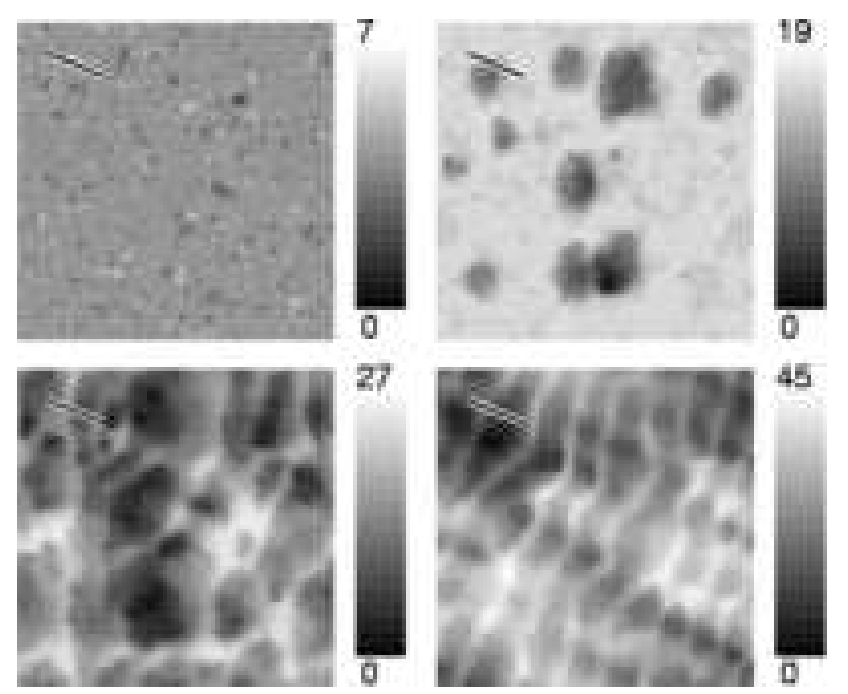

Figure 6.11: Surface profiles of region I (relatively smooth) of Fig. 6.9, $\sigma=1$, $\mu=1$. From top-bottom, left-right, $t=3,20,40$, and 90 . The bar denotes the ion beam direction. The topography evolves from a relatively smoother surface, at early times, to hole topography at intermediate times, and finally, to ripples at longer times.

Region I: rough surface [see Fig. 6.10(I)] which, as time increases, evolves to a hole topography. The sizes of the holes grow and finally coalesce to a rippled topography at long times (Fig. 6.11). Note that the surface is rough here only in comparison to its initial flatness. It is actually smoother, relative to the other regions, as can be seen from the height scales (see also the calculation in Fig. 6.20).

Region II: holes are already prominent in this region [see Fig. 6.10 (II)]; here the number of holes increase with time, and again ripples are formed at long times, but at an earlier time than as in region I (not shown as separate figure). The number of holes decrease when increasing the sputtering depth 


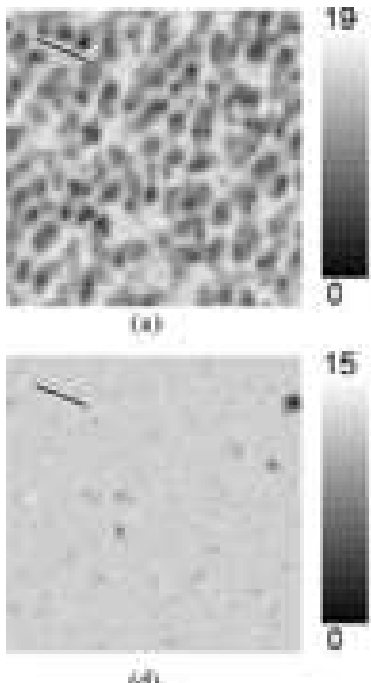

(d)
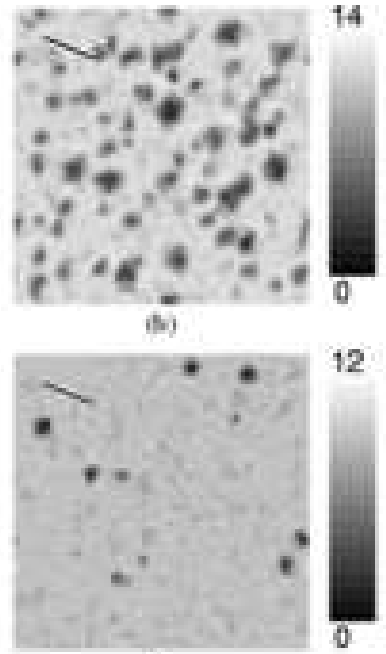

ies

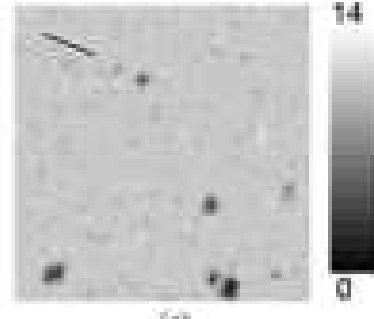

(c)

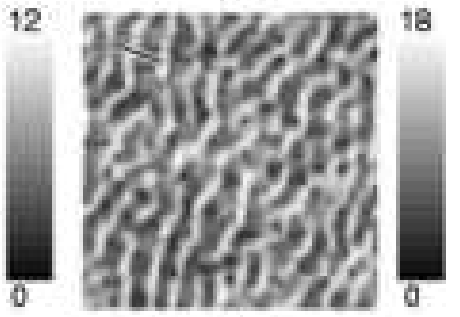

if

Figure 6.12: Surface profiles of region $I I$ (holes), $t=3$ ions/atom $(\sigma=1$, $\mu=1.5$ ). Top row; $\theta=50^{\circ}, a=5$ (a), $a=6$ (b), and $a=7$ (c). Bottom row; $a=6, \theta=40^{\circ}(\mathrm{d}), 45^{\circ}(\mathrm{e})$, and $60^{\circ}$ (f). In (a) - (c), the number of holes decrease with increasing penetration depth a, while in (d) - (f), the number of holes increase with increasing $\theta$, until ripple formation occurs at $\theta=60^{\circ}$ (f).

$a$ [Fig. 6.12 (a) - (c)]. On the other hand, if we vary $\theta$, the number of holes increase with increasing $\theta$ [Fig. 6.12 (d) - (f)]. Ripples can be formed here at this time $(t=3)$ if $\theta$ is increased beyond $\theta_{r} \approx 60^{\circ}$.

Region III: the ripple phase [82, 105]. Having observed in regions I and II that holes evolve into ripples with time, we studied this region from the very earliest times $(t=0-3)$ but found only very tiny holes, i.e not as pronounced as in region II, in the course of ripple formation (see Fig. 6.13). $\theta_{r} \approx 30^{\circ}$ in this region. Thus, comparing regions I, II and III, there seem to be two different processes of ripple formation. Ripples can be formed quickly by evolving directly from a slightly rough surface, or they can be formed slowly via the creation of holes, which coalesce to ripples at longer times. Note that in regions I and II, the resulting ripple wavelength is smaller than the size of the holes generated at smaller time, while in region III the ripple wavelength is larger than the tiny holes.

To summarize the dynamical behavior observed in regions I - III, for long (but finite) times ripples are formed everywhere, but region III grows at the expense of the two other regions. Region II represents only a short transient behavior. It might extend further to larger values of $\sigma$ in a very narrow range between regions I and III, but we cannot resolve this with our limited 

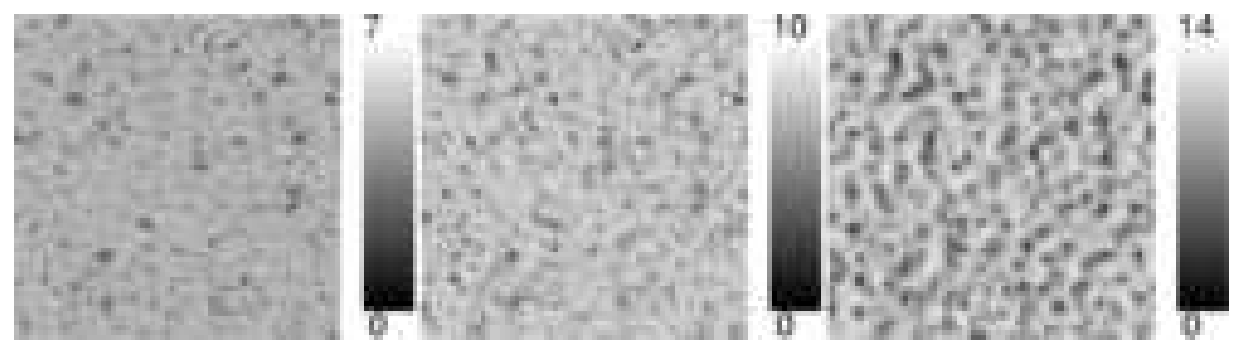

Figure 6.13: Surface profiles of region $I I I$, at very early times. From left-right, $\mathrm{t}=0.1,0.2$ and 0.5 ions/atom $\left(\sigma=3, \mu=1.5, \theta=50^{\circ}, a=6\right)$. The surface remains rough, i.e, no hole topography of the type shown in Figs. 6.11 and 6.12,

number of parameter combinations. Please note that at very long times, beyond the usual time scales accessible in standard experiments, nonlinear effects become more important and the ripples vanish [82, 105. Also, it was shown in the simulations of Ref. [82, that the ripples in this region rotate by $90^{\circ}$ with increasing fluence (the same result was obtained in the experiments of Ref. [85, and predicted in Ref. [66]).

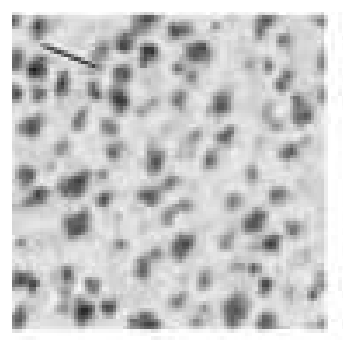

(a)
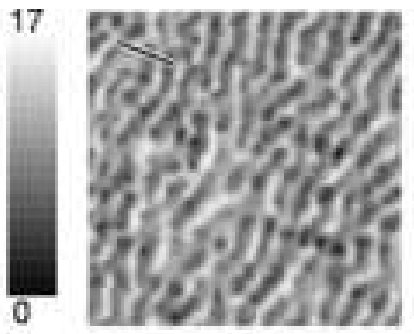

(b)

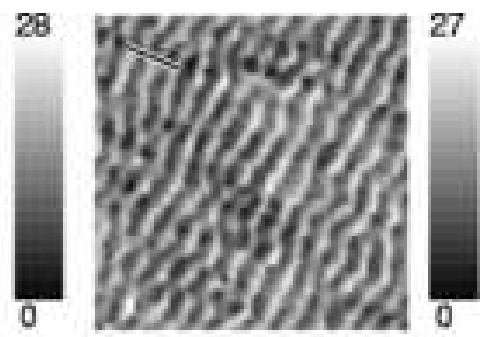

(c)

Figure 6.14: $\theta=30^{\circ}, a=6, \mu=1.5 ; \sigma=2$ (a); $\sigma=3$ (b); and $\sigma=5$ (c). Within region III, higher $\sigma$ result to lower $\theta_{r}$.

In the case of smaller angles, e.g. $\theta=30^{\circ}$ (Fig. 6.14), the hole region expands to $\sigma=2$, i.e, ripple formation is shifted to higher $\sigma$, at this typical time. Which implies ripple formation is shifted to later times (beyond typical experimental fluences) for lower angles of incidence. This indicates that there might be a lower critical angle $\theta_{r}$, below which no ripple formation happens even at long times. Such an effect has been observed in experiments 14] of Xe-ion sputtered Si, where below $\theta_{r}=40^{\circ}$ no ripples were found at finite but long ion fluences. For even smaller angles like $\theta=20^{\circ}$, we indeed do not observe ripple formation within the times (i.e. fluences) we can reach in our simulations. Note that a general statement about the existence 
of such an angle $\theta_{r}(\mu, \sigma)$ would require simulations up to very large times for all parameters studied here, which is beyond the numerical capacities. Hence, we remain with the statement that our numerical results indicate that such critical angles indeed exist, without the possibility to determine them precisely.

Region IV: consists of a mixture of dots and short ripples, which eventually give way to the dot "phase" (region V), as $\sigma$ increases. Hence, this region is an intermediate stage between regions III, V, and VI.

Region V: consists of dots. These dots are formed on some ripple-like structures oriented parallel to the ion beam direction, as discussed below in more detail. Noting that our model is a solid on solid model on a square lattice, the dots are not unsimilar to the QDs predicted by theory 70, 174] and observed in experiments [25, 26].

Region VI: consists of non-oriented structures exhibiting a typical length scale, but only a slight orientational preference parallel to the ion beam. This region, as mentioned above, is probably difficult to access in experiments.

\subsection{2 k-Space}

A closer look at the dot profiles of region V in Fig. 6.10 reveals the presence of some underlying large-scale structures. We now discuss this region and its adjacent regions III and $\mathrm{V}$ in more detail. The underlying structure is clearly seen in a 2D structure factor, $S(\mathbf{k})=\langle h(\mathbf{k}) h(-\mathbf{k})\rangle$. Where $h(\mathbf{k})$, the fourier transform of the height profile $h(\mathbf{r}, t)$, with mean $\langle h\rangle=\sum_{\mathbf{r}} h(\mathbf{r}, t) / L^{2}$, is given by Eq. (6.2). The 2D structure factor shown in Fig. 6.15 has been obtained from an average of 600 independent runs, for parameter along $\mu=4$ (top row), and along $\sigma=3.0$ (bottom row). In these diagrams the structure factor for the k-vector $\left(k_{x}, k_{y}\right)=(0,0)$ is displayed in the center and the corners represent the values for $\left(k_{x}, k_{y}\right)=2 \pi( \pm 1 / 8, \pm 1 / 8)$.

The case of $\mu=4.0$, when moving from small values of $\sigma$ to larger values (i.e. left-right in Fig. 6.9) is shown in the top row of Fig. 6.15 Small values of $\sigma$ fall within region VI, where we see a typical wavelength, but only a slight preferential orientation. This translates to a ring visible in the $S(\mathbf{k})$ plot. Note that there is a slight preference for an orientation parallel to the ion beam, being visible via two peaks in $S(\mathbf{k})$ at wave vectors perpendicular to the ion beam (see also the real-space profile, of region VI, in Fig. 6.10). When increasing $\sigma$, one moves into regionV. Here a line perpendicular to the ion beam emerges in the structure factor rather abruptly around $\sigma=1$. This line represents the underlying 1D structures parallel to the ion beam, being visible in Fig. 6.10. Note that the dots emerge on top of these structures; in the $2 \mathrm{D}$ structure factor their signal is too weak to be visible. 

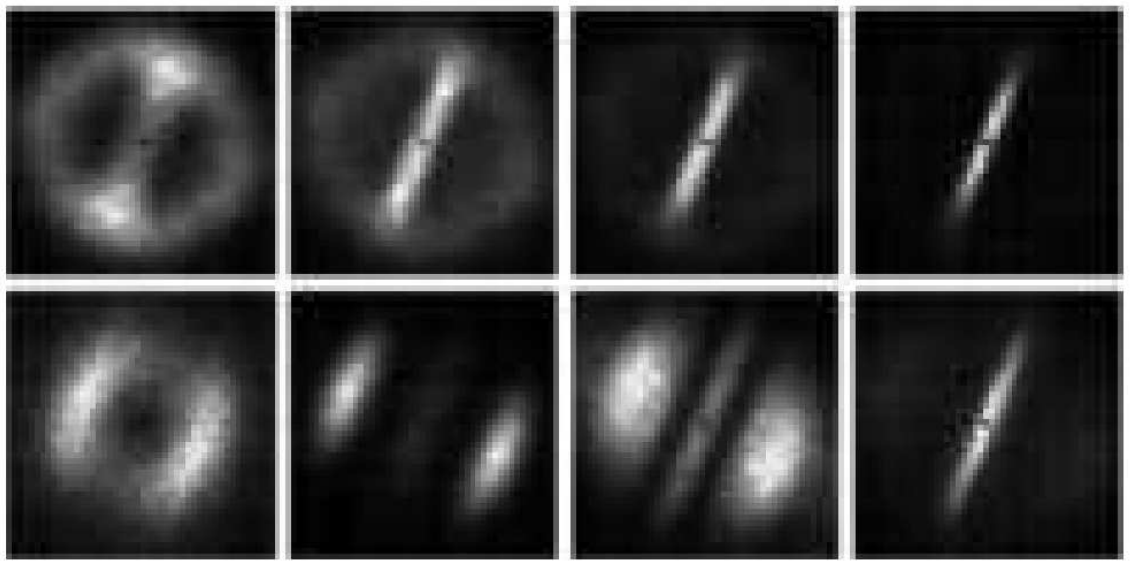

Figure 6.15: $2 \mathrm{D}$ structure factor $(\mathrm{L}=128)$. Top, along $\mu=4$ in Fig. 6.9 leftright: $\sigma=0.5,1.0,1.5$, and 3.0. Bottom, along $\sigma=3$, left-right: $\mu=0.5,1.0$, 1.5, and 3.0. In this diagram, the structure factor for the $k$-vector $\left(k_{x}, k_{y}\right)=(0$, $0)$ is displayed in the center, and the corners represent the values for $\left(k_{x}, k_{y}\right)=$ $2 \pi( \pm 1 / 8, \pm 1 / 8)$.

On the other hand, along $\sigma=3.0$, we initially see an orientation (bottom row of Fig. 6.15), spread around ion beam direction (for instance, at $\mu=$ 1.0 ), with a more restricted range of $k$ which is typical of the thin wobbly ripples. As $\mu$ increases, we move to the region $\mathrm{V}$ as discussed above, but we do not observe an abrupt change, because for a large range of values of $\mu$, ripples, dots and the underlying structures with parallel orientation, to ion beam direction, coexist (see below). To study the crossover from one region to the other in a quantitative way, it might be more instructive to look at order parameters which are numbers rather than the full 2D structure factor. We first define a quantity $Q=S^{m}\left(\mathbf{k}_{\perp}\right) / S^{m}\left(\mathbf{k}_{\|}\right)$, where $S^{m}\left(\mathbf{k}_{\|(\perp)}\right)$ is the maximum, for $\mathbf{k}$ parallel (perpendicular) to ion beam direction. This quantity detects the change of orientational order by comparing the relative intensities of the bright spots in Fig. 6.15 along the two directions. A separation of the $2 \mathrm{D}$ structure factor into regions, for the purpose of this analysis, is shown in Fig. 6.16.

Along $\mu=4$ from region VI into region $\mathrm{V}$ we expect a rather abrupt change of the behavior from the visual inspection of the $2 \mathrm{D}$ structure factor (top row of Fig. 6.15). The behavior of $Q$ when changing $\sigma$ at $\mu=4$ is displayed in the inset of Fig. 6.17. For small values of $\sigma$, i.e. in region VI, there is only a slight preference of structures parallel to the ion beam 


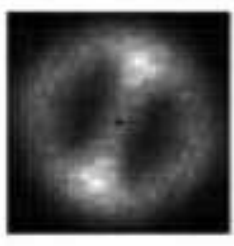

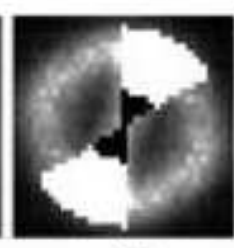

(1)

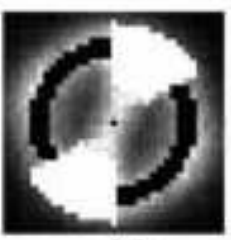

(r)

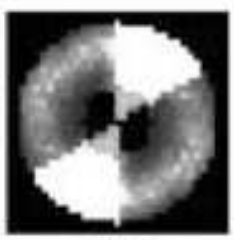

(b)

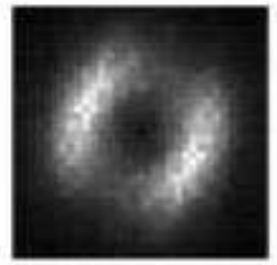

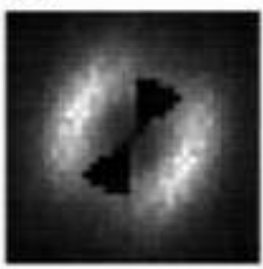

(

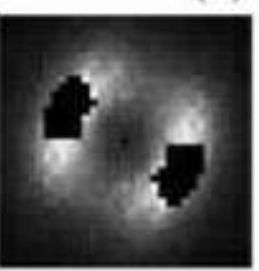

(II)

Figure 6.16: Separation of the 2D S(k) (first image top and bottom) into regions (in black from second image), for the purpose of our analysis. The letters l, r, b, denote line, ring and background, respectively; and the symbols, $\perp$ and $\|$, denote the perpendicular and parallel directions, respectively; as referred to in the text. The white regions are excluded from the analysis.

(corresponding to $\mathbf{k}_{\perp}$ ) which leads to small values $Q$. When going beyond $\sigma=1$, i.e. when moving into region $\mathrm{V}$, the domination of the longitudinal structures increase, leading to a growth of $Q$.

We, furthermore, want to go beyond studying the height of peaks of $S(\mathbf{k})$ by examining the presence or absence of a typical length scale in the system. This length scale is visible in the $2 \mathrm{D}$ structure factor via bright spots (oriented) or a bright ring. For this purpose, we also look at the relative weights

$$
q_{x / y}=\frac{\int_{A_{x}} S(k) d \mathbf{a}}{\int_{A_{y}} S(k) d \mathbf{a}},
$$

of certain areas in the $2 \mathrm{D}$ structure factor, where the integration $d \mathbf{a}$ extends over areas $A_{x} / A_{y}$ of $\mathbf{k}$-points. The indices $x, y=l, r, b$, refer to $\mathbf{k}$ values on the line, ring and background (outside line and ring) respectively, as shown in the upper part of Fig. 6.16. The white regions, in this figure, are excluded from our analysis, because they contain a superposition of $\mathbf{k}$ 's on both the line, and the ring. A plot of $q_{r / b}$ is shown in Fig. 6.17, other combinations (not shown) yield results within formation not going beyond the study of $Q$. For $q_{r / b}$ we observe an abrupt change around $\sigma=1$ with the ring becoming 


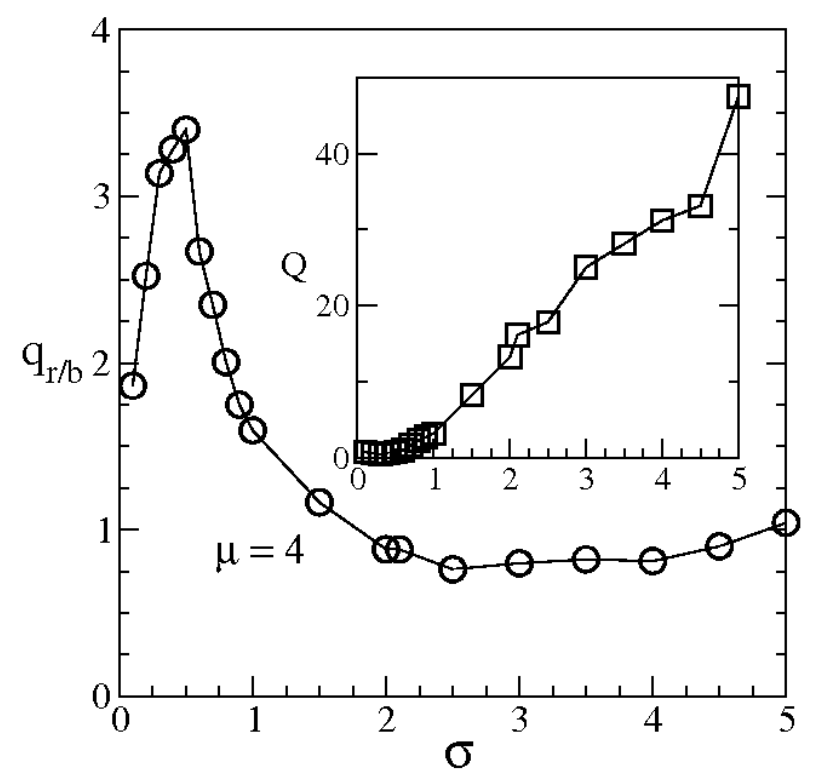

Figure 6.17: Plot of the quantities $q_{r / b}$ and $Q$ (inset) as functions of $\sigma$, along $\mu=4.0$, as described in the text. The lines are to guide the eyes.

almost indistinguishable $\left(q_{r / b} \approx 1\right)$ from the background for $\sigma>1$, signaling the disappearance of a typical length scale around $\sigma=1$. Note that $q_{r / b}$ stays almost constant for the full region $\mathrm{V}$, in contrast to the behavior of $Q$.

Now, we turn to the behavior along the $\sigma=3$ line, i.e. when moving from region I, into region III, then IV and finally $\mathrm{V}$. The behavior of $Q$ as a function of $\mu$ (bottom row of Fig. 6.15) is displayed in the inset of Fig. 6.18. The crossover, around $\mu \approx 2$, from the ripple region (II) where $k_{\|}$wave vectors dominate $(Q<1)$ to the region $\mathrm{V}$ where $k_{\perp}$-structures dominate is clearly visible. The same result is obtained, when again studying not only peaks, but integrated structure factors over certain selected areas. Hence, we also study k-vectors parallel and perpendicular to the ion beam $(x, y=\|, \perp)$, as shown in the lower part of Fig. 6.16. Also $q_{\perp / \|}$ exhibits a strong growth for $\mu>2$, i.e. when moving into region III.

Finally, a study of $q_{l / r}, q_{l / b}$ and $q_{r / b}$ as functions of $\sigma$, shown in Fig. 6.19, again confirms the loss of a certain length scale when moving into region $\mathrm{V}$. This is again visible via the disappearance of weight on the ring in the $2 \mathrm{D}$ structure factor with respect to the background [see Fig. 6.15. In any case, we have mainly looked at snapshots at time $t=3$, corresponding to typical experimental fluences of $10^{15}$ ions $/ \mathrm{cm}^{2}$ (as already stated above). Hence, the 


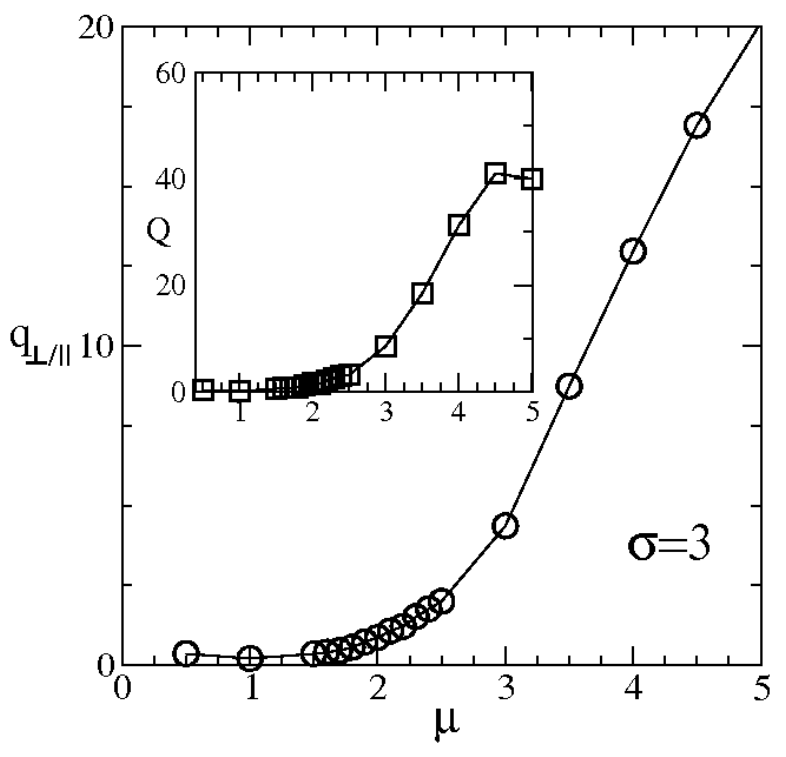

Figure 6.18: Plot of the quantities $q_{\perp / \|}$ and $Q$ (inset) as a function of $\mu$, along $\sigma=4.0$, as described in the text. The lines are to guide the eyes.

location of the exact boundaries between the different regions, is not our main focus. We only want to demonstrate, that indeed order-parameterlike functions can be defined. The full information is contained in the 2D structure factors.

Next, we consider the predictions of the continuum theory, as pertains to this study. In the asymptotic limit, the morphology of sputtered surfaces may be described by the noisy KS Eq. 3.23. Note that erosion of surface material tends to maximize the exposed area, so-called negative surface tension, hence, for the sputtering phenomena, this instability constraints the coefficients $\nu_{x}$ and $\nu_{y}$ to be negative. At early times, the local slopes are small enough in most of the regions for us to ignore the nonlinearities; we are therefore left with a noisy Bradley-Harper equation [41]. Henceforth, in our calculation of the coefficients of the continuum equations, we set $J p=1$ (see Ref. [39]). A plot of the coefficients $\nu$ for our parameter range, along $\sigma=3$, is shown in Fig. 6.20. $\left|\nu_{x}\right|$ and $\left|\nu_{y}\right|$ are nonzero almost always, which implies the presence of two length scales. Hence, according to the linear continuum theory, ripples parallel and perpendicular to the ion beam direction are always present in the system; the one observed is the one for which $|\nu|$ is highest; i.e, with the 


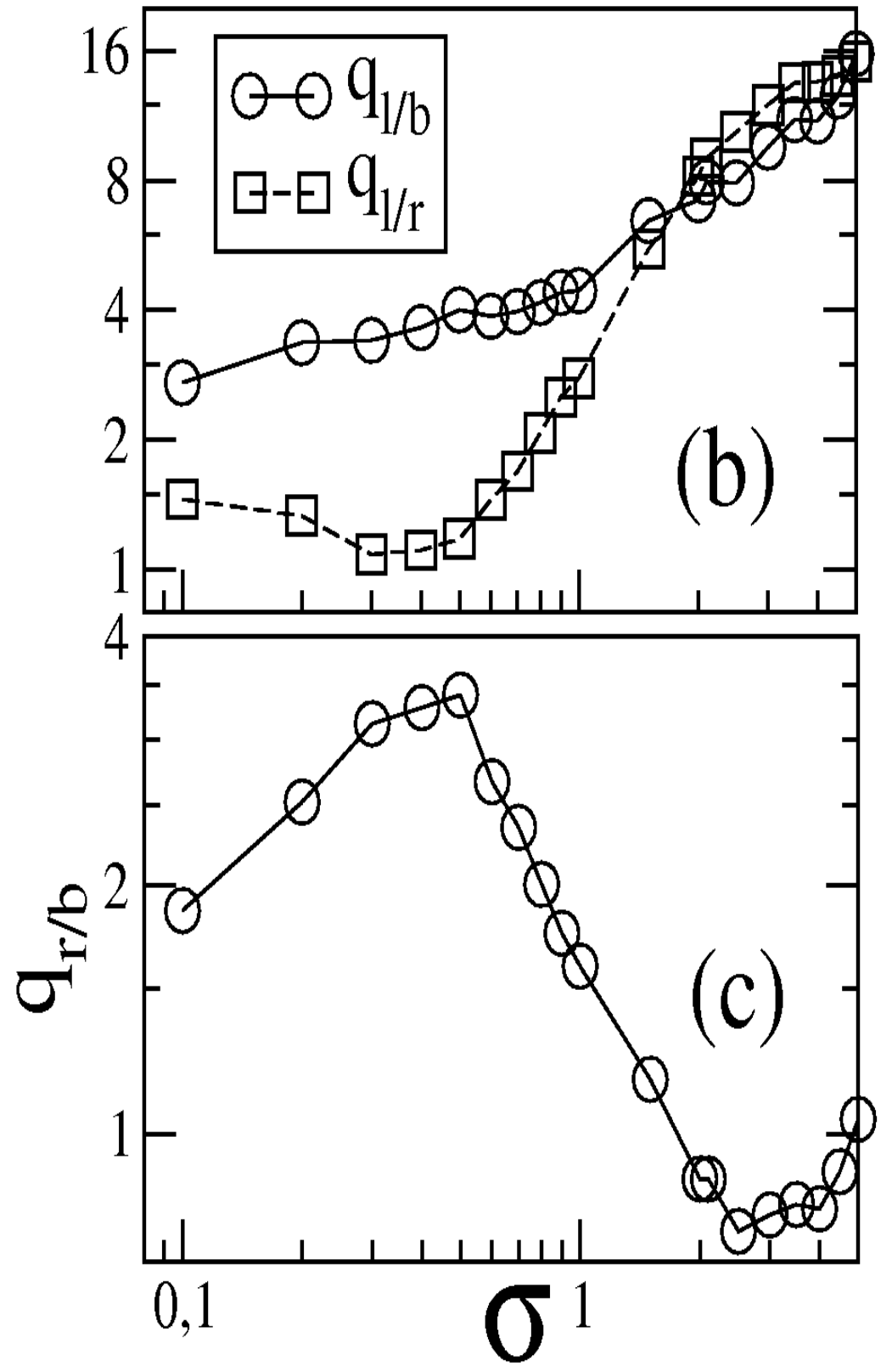

Figure 6.19: Plot of the quantities $q_{l / b}$ and $q_{l / r}$ (a), and $q_{r / b}(\mathrm{~b})$, as functions of $\sigma$, along $\mu=4.0$. The lines are to guide the eyes. 


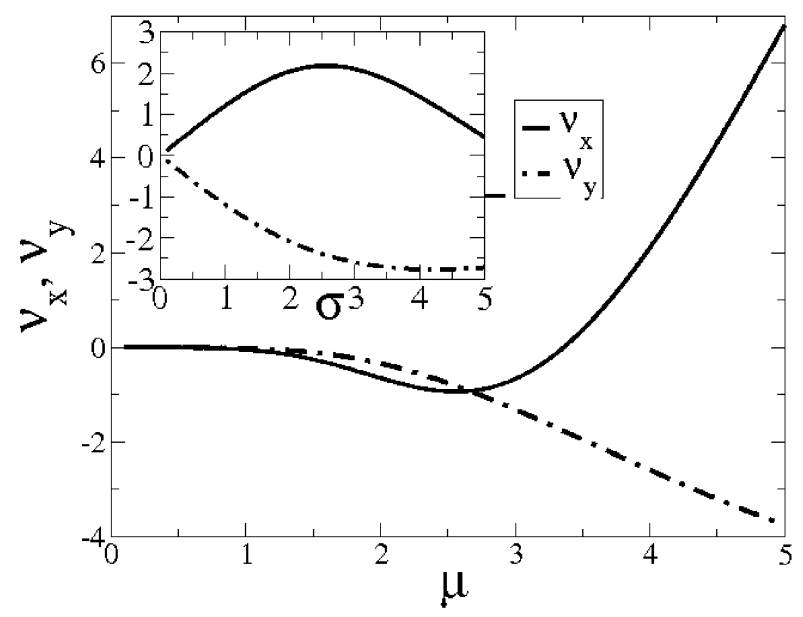

Figure 6.20: Plot of the surface tension coefficients $\nu_{x}$ and $\nu_{y}$ [of Eq. (3.23)] versus $\mu$, along $\sigma=3.0$, for our simulation parameters. In the inset is the plot of $\nu_{x}$ and $\nu_{y}$ versus $\sigma$, along $\mu=4.0 . \theta=50^{\circ}, a=6.0$.

highest amplitude-growth rate

$$
R=|\nu| k^{2}-D k^{4} \text {, where } \nu=\min \left(\nu_{x}, \nu_{y}\right) .
$$

We now consider the behavior along the above discussed $\sigma=3$ and $\mu=$ 4 lines within the linear theory. In Fig. 6.20 the values of $\nu_{x}$ and $\nu_{y}$ are shown as functions of $\mu$ (main plot) and $\sigma$ (inset) along these lines. For the case $\mu=4$ (see inset), we observe that $\nu_{x}>0$ for all values of $\sigma$, hence the preferential orientation is always parallel to the ion beam, as observed. To understand the crossover from region VI to region $\mathrm{V}$, perhaps one has to consider nonlinear terms. For the $\sigma=3$ line, we observe $\left|\nu_{y}\right| \approx\left|\nu_{x}\right|$ for $\mu<$ 1 , which is compatible with the behavior in region I, where no preferential orientation is observed. For $1 \lesssim \mu \lesssim 2.5,\left|\nu_{x}\right|>\left|\nu_{y}\right|$ which implies the dominance of ripples with $k_{\|}$(i.e, with wavelength $\lambda=2 \pi \sqrt{2 D /\left|\nu_{x}\right|}$ ), as we have seen in region III and partly in the crossover region IV. In region IV ripples are still present, but dominate less.

For $\mu$ above this range, the structures with $k_{\perp}$ dominate. However, the positive value of $\nu_{x}$ for higher $\mu$ is contrary to our results, since it implies a preferential smoothening along the parallel direction. We have not observed such smoothening, here probably nonlinear effects are more important. If we consider the maximum of $\left|\nu_{x}\right|$ and $\left|\nu_{y}\right|$ for the region where $\nu_{x}<0$ and/or $\nu_{y}<0$, then we see that this maximum is obtained in region $\mathrm{V}$ for large $\mu$. 
Since larger $|\nu|$ implies a higher tendency to maximize the exposed surface area, i.e. increased roughening, one should expect rougher surfaces in region $\mathrm{V}$. Indeed we observe that region $\mathrm{V}$ is rougher than e.g. region III, as we shall see in the next section.

\subsubsection{Roughness, and growth exponent}

In this section we want to examine the fluctuations of the interface width. But, before we proceed, we should recall that regions I and II eventually evolve to a similar rippled topography as in region III; region IV is an intermediate stage; and VI is hardly experimentally accessible. Therefore, we are interested in two different scaling regions, i.e, arising from ripple-yielding, as well as dot yielding parameters. We use the finite-size scaling behavior of the surface width/roughness, as described in section 3.2 .

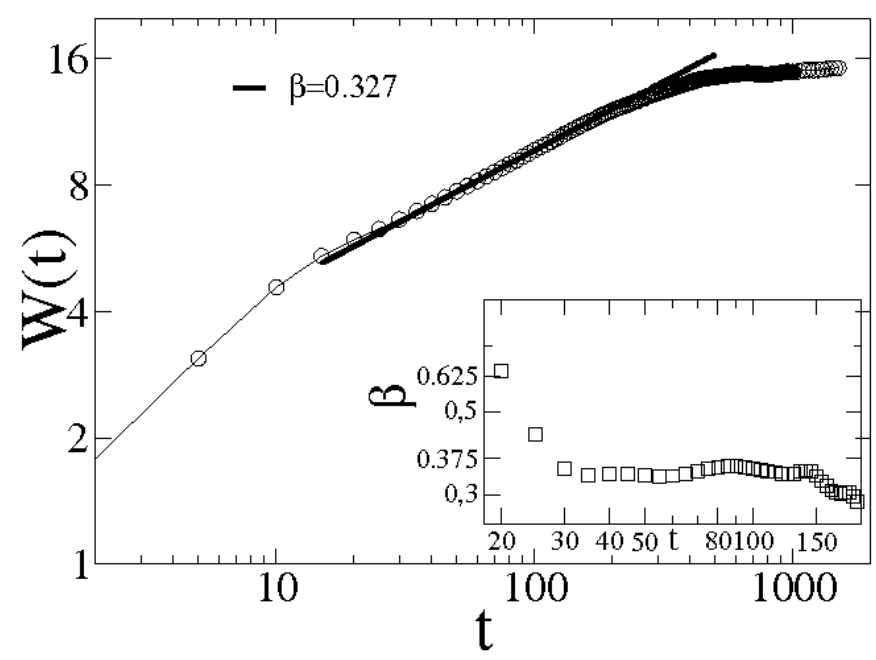

Figure 6.21: Surface width $W$ versus time, $\sigma=3, \mu=1.5$ (region $I I I$ ), $\theta=50^{\circ}$. In the inset is a plot of the consecutive $\beta$ s for $t=20-200$, where the lower and upper cutoffs are seen to occur at $t=35$ and 150 respectively.

In the following roughness analysis, we rescale the time unit such that $L^{2}$ particles are eroded in unit time, which is analogous to the measure of time in simulations of epitaxial growth on vicinal surfaces. We obtained our scaling exponents from an average of 600 independent runs. Fig. 6.21 is a plot of the surface width $W(t)$ for $L=128$, we determine the actual scaling region of $\beta$ from the consecutive slopes [1] of $W(t)$ for $t>L^{z}$; shown in the inset 
of Figs. 6.21 and 6.22 where a fit to this region gives $\beta=0.327 \pm 0.001$, and $\beta=0.665 \pm 0.003$ for the topographic regions (see Fig. 6.9) III and $\mathrm{V}$ respectively; i.e., the long-time scaling behavior for sputtering conditions coinciding with the respective regions. Please note that the data shown in the insets of Figs. 6.21 and 6.22 were only used to determine the correct scaling region, for the actual fit in the main plots.

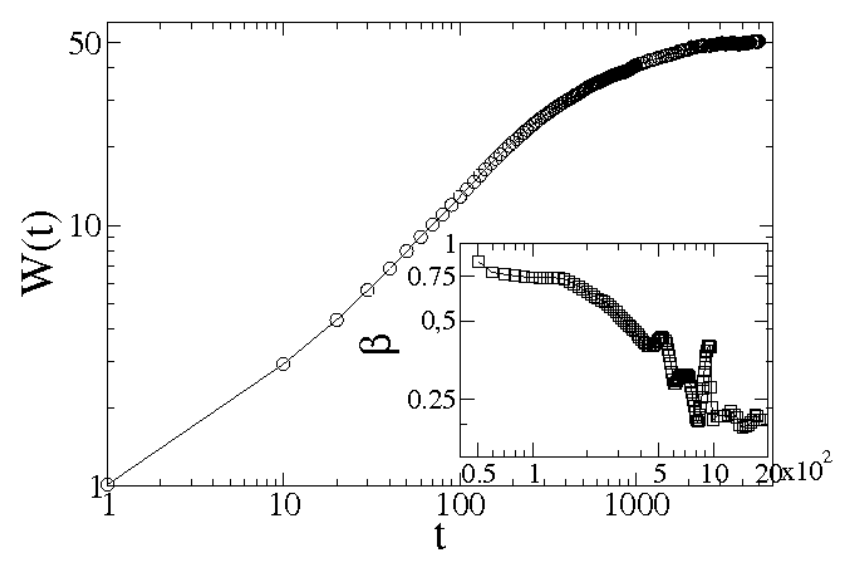

Figure 6.22: Surface width $W$ versus time, $\sigma=5, \mu=5$ (region $V$ ), $\theta=50^{\circ}$. The inset shows two scaling regions $t=40-140$ and $t=1000-1800$, which yields $\beta=0.665$ and 0.137 respectively.

Our exponents are different from the scaling, $\beta=0.25$, of the KPZ equation in $(2+1)$ dimensions [1, 112, which shows that for these parameter choices, either one or both of the negative surface tension coefficients, in the noisy KS equation, do not change to positive values in a renormalization group analysis. That is, if indeed, this atomistic model which is known to accurately describe the morphology of sputtered surfaces at early and long times [82, 85, 105], can be represented by the noisy KS equation, in the asymptotic limit, as expected for the sputtering phenomenon.

\subsection{Rotated Substrate}

In this section we consider the effect of sample rotation on the topographies observed in the previous section, as mentioned in the introduction to the chapter.

Sample rotation is known to affect the topography of concurrently sputtered surfaces. For instance, a number of experiments have shown that ripples 
can be removed or suppressed by simultaneous sputtering and sample rotation [29, 72]; so called Zalar rotation. This has been explained theoretically [50, 73], as described in section [3.7, with the requirement that the angular speed be high enough for the surface topography to be different from the non-rotated case. Such rotation leads to a remarkable smoothening of the pre-roughened surface. It has been shown that concurrent rotation and sputtering leads to oblique ion-incidence quantum dot formation [30, 74]. In the experiments of Ref. 30, described in chapter 2, oriented cones were observed for off-normal incidence without sample rotation, whereas, a highly regular hexagonal pattern of cones (or mounds) was found with sample rotation.

In section 6.2 we showed the possibility of dot formation without sample rotation. In this section, we present results which indicate that sample rotation removes the underlying one-dimensional structures observed in section [6.2, in the dot region, thus smoothening the substrate upon which the dots form. In addition to this, with rotation, we found that all other regions evolve to non-oriented structures with time. We have already shown, in section 5.1.2. how sample rotation may be implemented in the HKGK simulation model, following the insight gained from the continuum theory of rotated surfaces [50, 73]. As in the previous section, the continuum theory is employed to understand the results presented here.

\subsubsection{Profiles and discussion}

Here, we consider the topographic regions in Fig. 6.9, along $\sigma=1,3$, and 5, under simultaneous rotation; i.e, in order to see the effect of sample rotation on all the observed topographies. The obtained profiles are shown in Fig. 6.23. for samples rotated from the onset of sputtering (in all profiles shown in this section, $\left.k_{B} \mathrm{~T}=0.1 \mathrm{eV}\right)$.

As seen from this figure, no anisotroppy can be found with substrate rotation, as expected from the theory. However, hole formation is not suppressed, since we still get the same holes observed in section 6.2 in the hole region. This is not surprising, since the continuum theory predicts roughly equal erosion rates along both directions for parameters in the hole region (see Fig. 6.20), hence there is no anisotropy to be destroyed. Also, with rotation (as seen in Fig. 6.23), oriented ripple patterns in region III are replaced by non-oriented structures, and the one-dimensional structures in region $\mathrm{V}$ are no longer present. In order to see this more clearly we consider the surface in Fourier space, i.e, using the 2D structure factor. This is shown in Fig. 6.24,

According to the continuum theory (see section 3.7), although the broken symmetry that arises from tilting the ion beam to the surface normal is still present (i.e, $\nu_{x}$ may not equal $\nu_{y}$ ), it is no longer relevant in creating an 

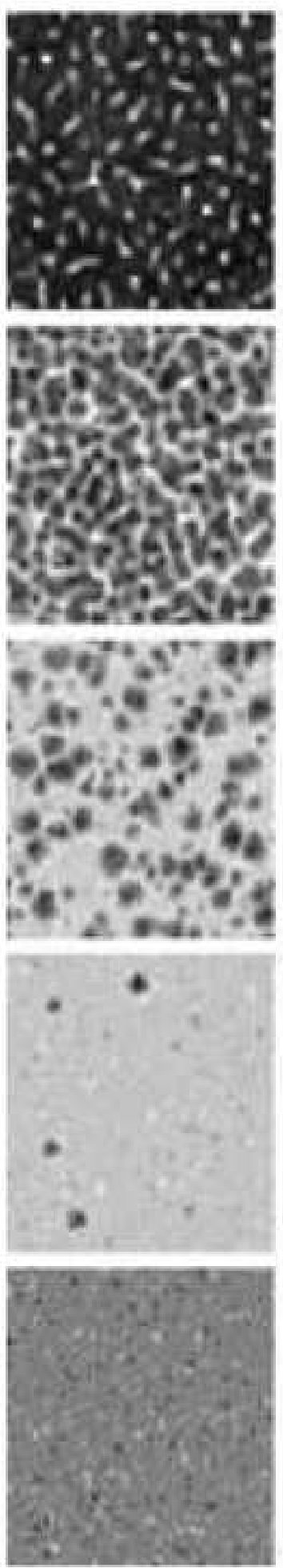

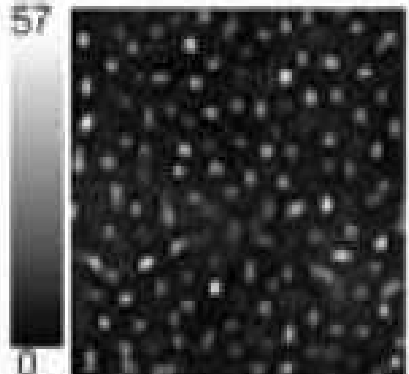

20
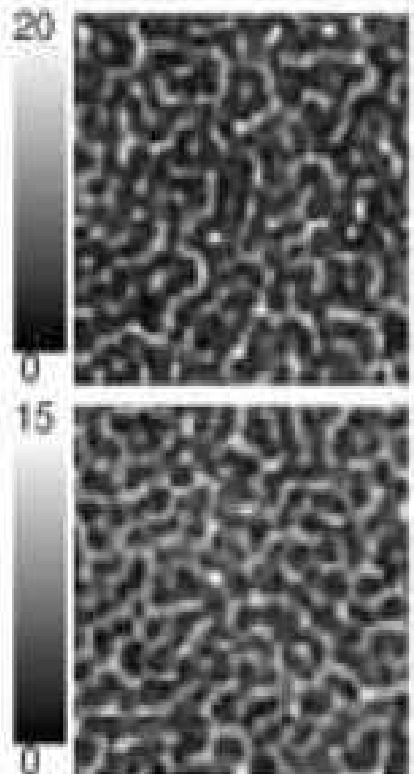

9

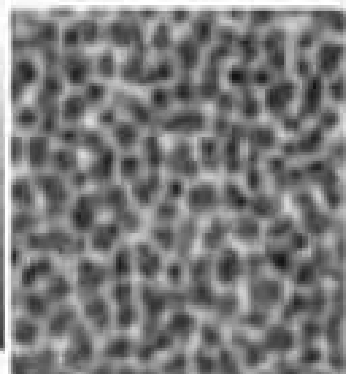

4

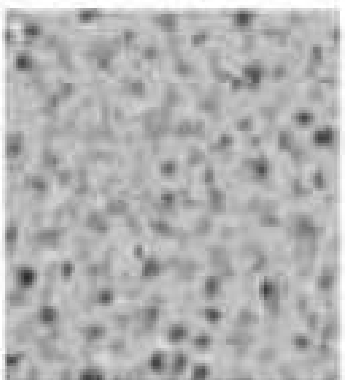

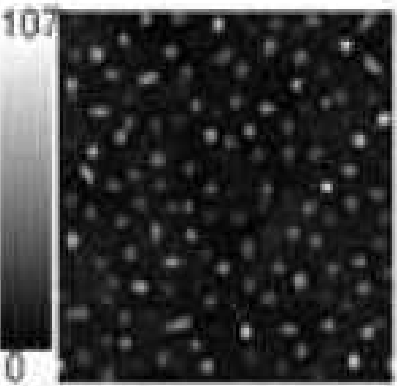

147

34
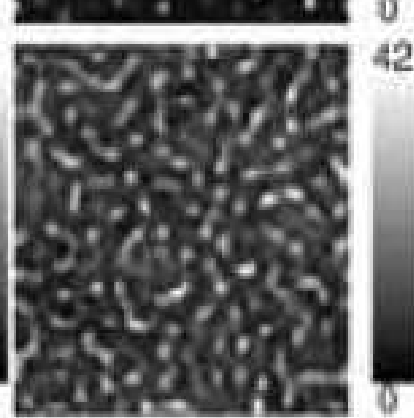

42
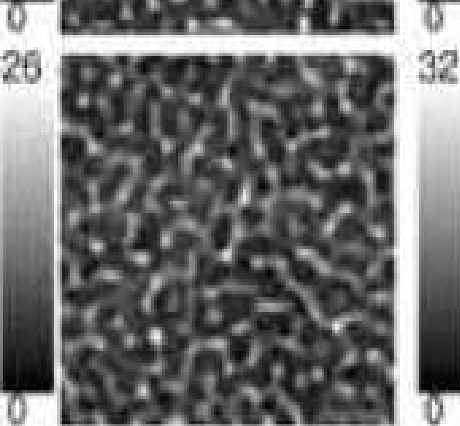

32
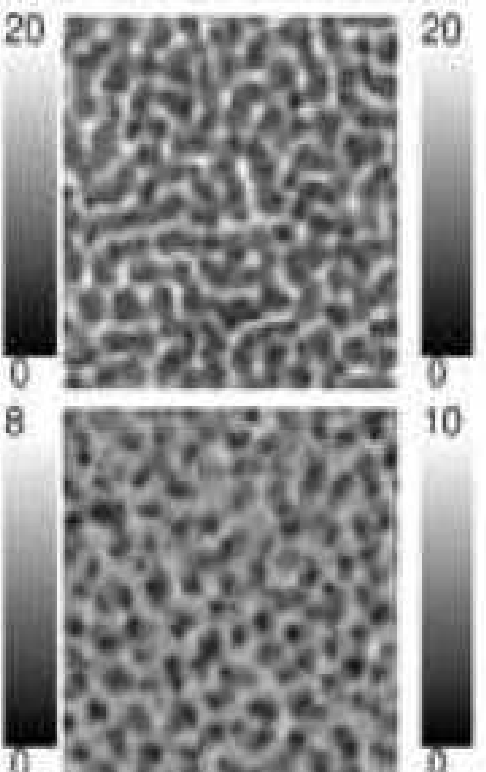

Figure 6.23: Profiles obtained from simultaneous sputtering and rotation. $t=$ $3, a=6, \theta=50^{\circ}$. Left - right columns: $\sigma=1,3$, and 5, respectively. Top row bottom row: $\mu=5,2,1.5,1$, and 0.5 , respectively. 

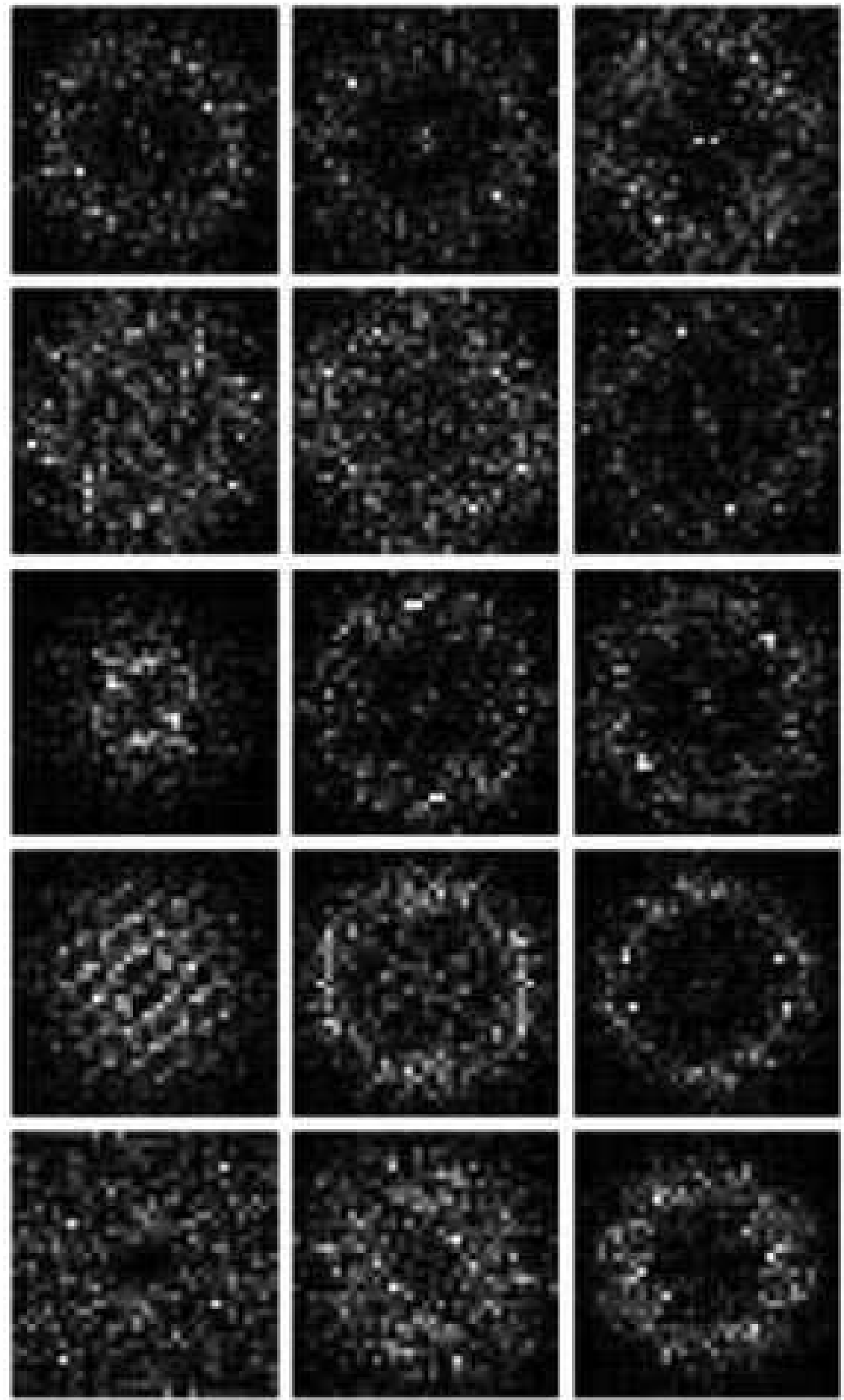

Figure 6.24: 2D structure factors of the profiles in Fig. 6.23, Left -right columns: $\sigma=1,3$, and 5, respectively. Top row - bottom row: $\mu=5,2,1.5,1$, and 0.5 , respectively. 
anisotropy in the system since there now exists an effective surface tension coefficient $\nu_{a v}=\nu_{x}+\nu_{y}$ which is the same along both directions (see Fig. 6.25 for a plot of these coefficients). The same argument applies to the nonlinear
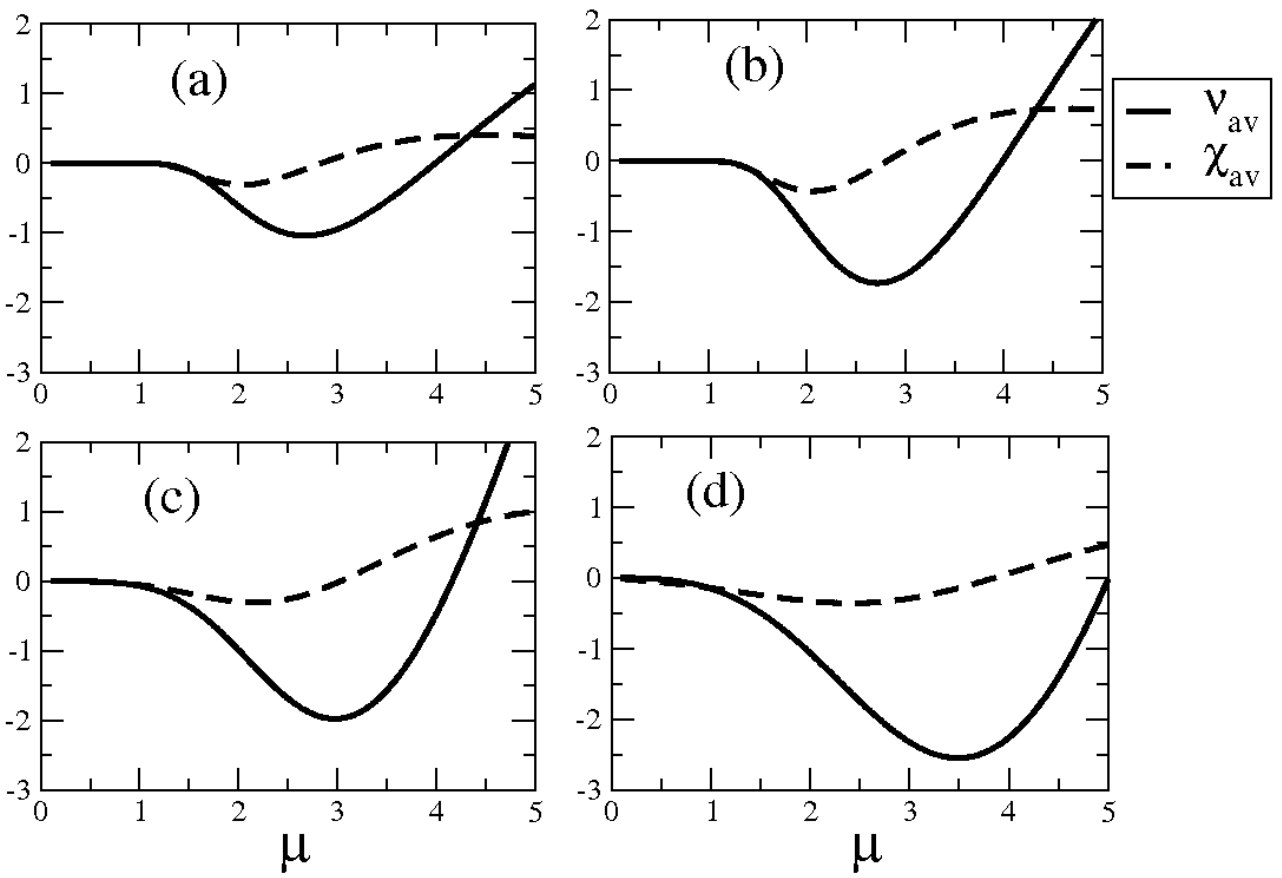

Figure 6.25: The coefficients, $\nu_{a v}$ and $\chi_{a v}$, of the iKS equation (3.36) for the rotated case, as functions of $\mu$. (a) $\sigma=0.5$, (b) $\sigma=1.0$, (c) $\sigma=3.0$, and (d) $\sigma=$ 5.0 .

coefficient $\chi_{a v}$. The result is that the surface roughens with time, with lower $\nu_{a v}\left(\nu_{a v} ; 0\right)$ corresponding to higher roughness; for instance, one sees that $\left|\nu_{a v}\right|$ increases in Fig. 6.25, as well as the increasing height difference on the greyscale charts on the profiles of Fig. 6.23. Surfaces in the parameter range for which $0>\nu_{a v} \gg-1$, are relatively smoother. With time, non-oriented structures emerge, as shown in Fig. 6.26, due to the isotropy; except for very low longitudinal straggle, $\sigma=1$, where no structure was found up to the longest simulation times. Since $\nu_{a v} \neq 0$, the surface is not randomly rough, which explains the presence of the non-oriented protrusions. Note that with increasing $\sigma$, the $\left|\nu_{a v}\right| \approx 0$ interval is reduced.

When the local surface slopes become significant (with prolonged sputtering), nonlinearities become relevant in (3.36). It has been shown that, where 
(a)

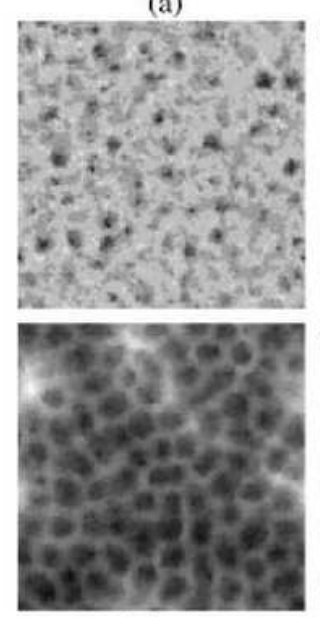

(c) (b)

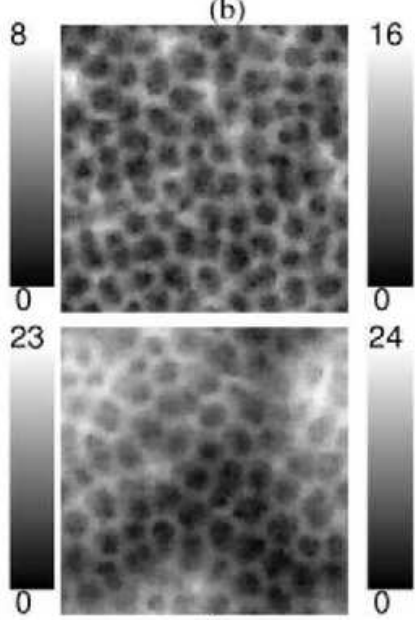

(d)

Figure 6.26: Time evolution of the rough topography equivalent to rotation of region I of Fig. 6.9, $\sigma=3, \mu=0.5$. (a) -(d): $t=3,40,90$, and 150, respectively.

ion induced effective surface diffusion is the dominant relaxation mechanism, and for $\theta=0$, crossover to the nonlinear regime either gives rise to dot formation (if $\chi_{a v}>0$ ), or hole formation (if $\chi_{a v}<0$ ). This is consistent with our results, where we found dots for the parameters at which $\chi_{a v}>0$ in Fig. 6.25 (i.e, for $\mu \gtrsim 3$ ). But, though $\chi_{a v}<0$ for the region where we found the holes, hole formation is not as widespread as Fig. 6.25 seems to indicate. Moreover, the hole topography eventually evolves to non-oriented structures at long times (see Fig. 6.27). For non-oriented structures, simulations at longer times reveal only slight changes in the structures; no dot formation (see Fig. 6.28). Also, prolonged sputtering of the dot region, with rotation, only seems to affect the height distribution of the dots. The profiles are shown in Fig. 6.29. Fig. 6.25 indicates a change of the effective negative surface tension coefficient $\nu_{a v}$ to positive values, which implies a smoothening, and dynamic scaling that falls into the KPZ universality class; i.e, as predicted by [50]. This smoothening is exhibited by our results, i.e, in the removal of the rough 1D structures by sample rotation. But this figure (i.e, the positive $\nu_{a v}$ in Fig. 6.25) also imply that the sputtering process itself no longer maximizes the exposed surface area, but instead, tends to minimize it. 


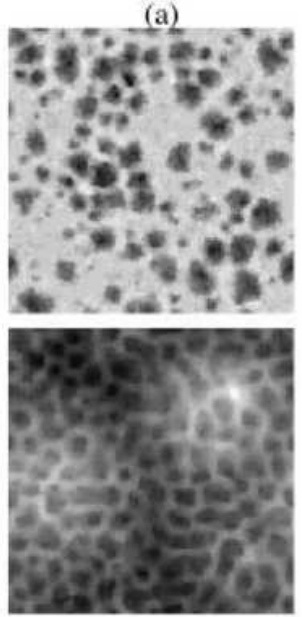

(c)

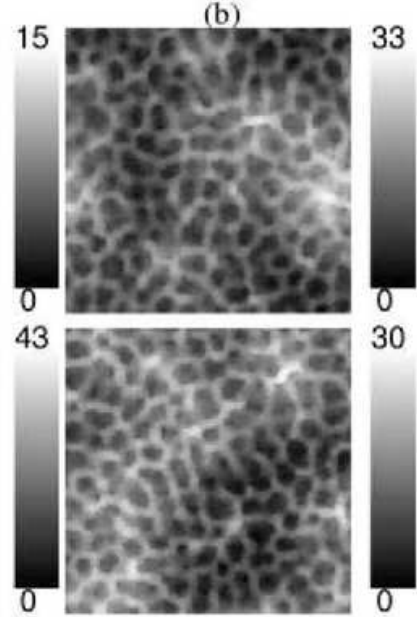

(d)

Figure 6.27: Time evolution of the hole topography in region II of Fig. 6.9, with sample rotation. $\sigma=1, \mu=1.5$. (a) - (d): $t=3,40,90$, and 150, respectively.

(a)

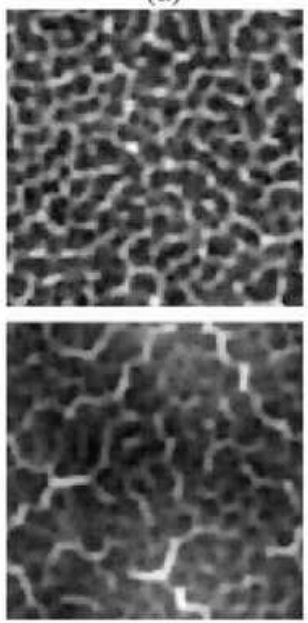

(c)

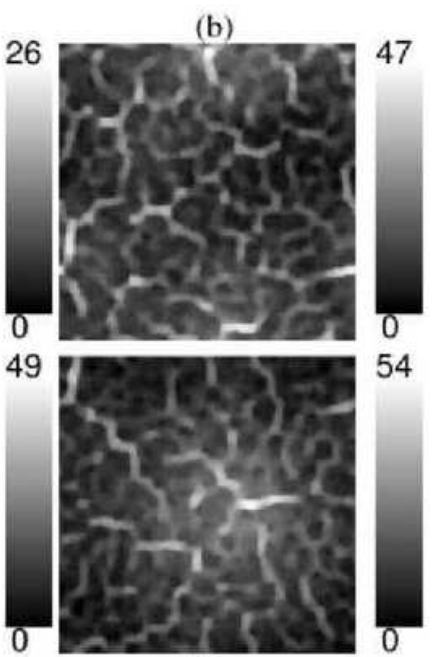

(d)

Figure 6.28: Time evolution of the non-oriented structure equivalent to rotation of region III in Fig 6.9, $\sigma=3, \mu=1.5$. (a) - (d): $t=3,40,90$, and 150, respectively. 


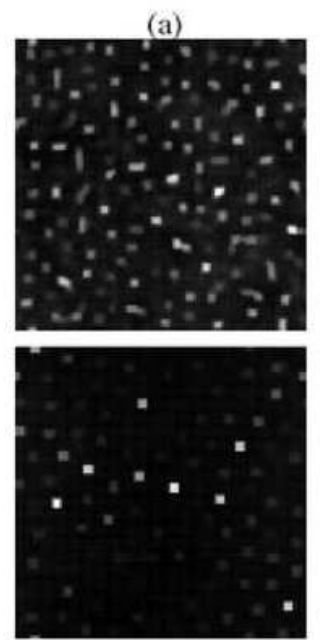

(c)

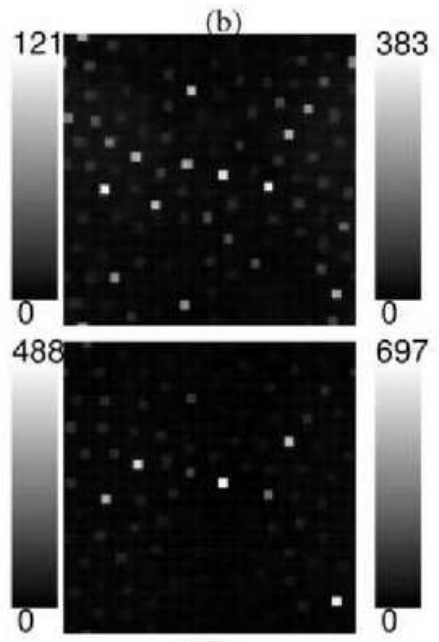

(d)

Figure 6.29: Time evolution of the dot structures equivalent to rotation of region $\mathrm{V}$ in Fig. 6.9, $\sigma=3, \mu=5$. (a) - (d): $\mathrm{t}=3,90,150$, and 250, respectively. 


\section{Every new beginning comes from some other beginning's end.}

Seneca

\section{7 \\ Conclusions}

When a solid surface at nanometer length scales is exposed to a uniform flux of ions (of the order of $10^{15}$ ions $\mathrm{cm}^{-2} \mathrm{sec}^{-1}$ ), for time scales of the order of minutes, surface patterns or structures of important technological applications may emerge. The mechanisms governing the surface morphology are well understood, from the continuum theory, as a result of the interplay between two competing processes. The roughening processes of surface erosion, which tends to maximize the exposed surface area. And the smoothening processes of surface material transport which is driven by the tendency to minimize the exposed area. In other words, sputtering energizes the surface, and thus leads to the erosion of surface material. Whereas, surface diffusion tends to minimize the surface energy. If the ions are incident normal to the surface, erosion is isotropic and the surface develops a cellular structure which, after prolonged sputtering, may give rise to surface protrusions (dots), or basins (holes), depending on the sputtering conditions. The dynamic scaling of the surface height fluctuations is expected to belong to the isotropic Kuramoto-Sivashinsky universality class.

With oblique incidence, however, surface particle removal is anisotropic, and, since the erosion velocity is tilt dependent and the sputter-yield is curvature dependent, the sputtering rate along a parallel direction to the ion beam direction is different from that along a perpendicular direction. Hence, for small incidence angles, surface particles self-organize into periodic patterns with wave-vector parallel to the ion beam direction. Whereas, for incidence angles close to grazing, the wave vector of the ripple-like structures is perpendicular to the ion beam direction. The dynamic scaling, in this case(i.e. $\theta>0$ ), is that of the anisotropic KS equation. The wavelength of the ripple structures can be calculated from the continuum theory. Initially, the ripple amplitude grows exponentially with time, but later stabilizes, when the local surface slopes become significant enough for nonlinearities to be rele- 
vant, either to a steady-state value or to a much slower growth rate. There are numerous experimental and computer-simulation corroboration of the predictions of the continuum theory.

Among the several predictions of the linear continuum theory is that, due to the tilt dependence of the erosion rate, ripples should propagate with a constant velocity, parallel to the surface, and along the ion beam direction. Also, the wavelength of these ripples is independent of time. Both of these predictions have been contradicted by experiments. In the experiments 22 , 38, the ripples have been found to coarsen with time, i.e. $\lambda=\lambda(t)$. But only power-law behaviors in the wavelength-time relationship have previously been found, to our knowledge. Also, the ripple velocity was found to be nonuniform with time.

We have studied the surface sputtering phenomena by means of Monte Carlo simulations, in relation to the continuum theory and experiments. When the model was introduced to the literature of surface sputtering phenomena, the crucial role of surface diffusion mechanisms, on the resulting surface morphology, was emphasized; while using the Hamiltonian and the Wolf-Villain surface diffusion models. It is believed that surface diffusion is a thermally activated process, and the surface material transport is not limited to a single iterative step (i.e unlimited mobility); driven only by the need to minimize the surface energy. Thus in this study we mainly focus on the thermally activated Arrhenius surface diffusion model. But, we first consider both unlimited mobility models in our study of ripple propagation and coarsening, where we found the $n=2$ Hamiltonian model to be qualitatively similar to the Arrhenius model (without Schwoebel barriers).

We summarize our results in the following sections.

\subsection{Ripple coarsening and motion}

We have obtained the formation and propagation of the ripples with both diffusion mechanisms used in turn. Furthermore, we have obtained the same trend in the behavior of ripple velocity and wavelength as observed experimentally and predicted theoretically. But, in addition to the experimental results, we find a drastic change in the ripple coarsening and propagation as temperature increases; for instance we found deviations from power-law into exponential or inverse-law behavior in the wavelength-time relationship, and in addition, the ripples first stop moving before vanishing completely. We find that, at very high effective temperatures, the behavior of the ripple velocity is characterized by two regions, separated at the transition time. In the first region it is constant and in the second region it decreases rapidly 
to zero. Between the two regions a power-law dependence can be observed for some small time interval. Whereas, around so-far experimentally realized temperature, the velocity-time relationship obeys a power-law.

Furthermore, at high effective temperatures, the wavelength increases exponentially with time in the thermodynamic diffusion model(and in the Arrhenius diffusion model without the Schwoebel term) and obeys an inverse law for the Arrhenius model including the Schwoebel barrier. An experimental verification of our higher temperature results has recently been provided in Ref. 85, where the ripple wavelength was found to increase exponentially with the sputtering time at elevated temperatures $\left(500-700^{\circ} \mathrm{C}\right.$, and $657^{\circ} \mathrm{C}$ in Ref. [113]) on ion patterned $\mathrm{Si}(111)$ surfaces.

In addition, we find further strong dependencies on the effective substrate temperature; as the temperature increases the magnitude of the velocity also increases. The transition time between constant and decreasing velocity was found to decrease with increasing temperature. Our results indicate an approach towards a saturation behaviour of velocity or wavelength with increasing effective substrate temperature, where the wavelength is expected to become time independent. However, this may happen at an unphysically high temperature.

Strong nonlinearities seem not to be responsible for the observed velocity dispersion, since we have observed the same behavior with this sputtering model which at most only has weak nonlinearities.

\subsection{Morphological regions and oblique inci- dence dot formation}

We have covered a range of experimentally relevant values of the collision cascade parameters in the HKGK model. And, in order to explain the results in the context of the continuum theory, we have considered the predictions of the theory beyond the circular-symmetric collision cascade case $\sigma=\mu=$ 1. We found a strong influence of the lateral straggle $\mu$ on the surface morphology. Below an upper bound $\mu \approx 2$, clear ripple formation is possible. Depending on the longitudinal straggle $\sigma$, ripples appear on different time scales, and through two different creation mechanisms. These ripples persist on intermediate time scales, but disappear again, as found in previous studies for one parameter combination, on longer times scales. Above the upper bound $\mu \approx 2$ nanosized islands were found for longitudinal straggle $\sigma \gtrsim 1.7$. These structures appear, from our studies, to be stable on longer time scales. Also, we found the possibility of different threshold $\theta_{r}$, above which ripple 
formation is possible, as in the experiment; our results indicating that high value of the longitudinal straggling result in lower value of $\theta_{r}$.

Comparison of our results with the continuum theory, reveal that dot formation is the result of the interplay between two (ripple-like) structures of perpendicular orientation: i.e, structures with wave vector either parallel or perpendicular to the ion beam direction. The tendency, due to the low incidence angle, is for the ripples with wave vector parallel to the ion beam direction to dominate. However, at high perpendicular straggle, ripplelike structures with wave vector perpendicular dominate by pinching off (or shortening), the ripples with wave vector parallel, into dots. This seems to suggest a possible structural difference between dots formed from off-normal incidence sputtering, where there is a broken symmetry as a result of tilting the ion beam to the surface normal, and dots formed from normal incidence sputtering, where there is no such broken symmetry.

We found that the scaling of the fluctuations in the surface height depends on the collision cascade parameters; whereby the roughness becomes saturated much earlier in the ripple region than in the dot region. Our results indicate the possibility of a yet unobserved dot topography; i.e $\theta>0$, without rotation.

\subsection{Rotated substrates}

Our simulations indicate that no new dots are formed with sample rotation. Rather, rotation destroys any anisotropy in the system. It has already been shown that the shape of the ion-induced collision cascade determines whether dots, or ripples, or other topographies occur; i.e, without sample rotation. For ripple yielding parameters, rotation destroys the anisotropy that leads to ripple formation, thus yielding non-oriented structures instead of ripples. For dot-yielding parameters, rotation enhances the isotropy in the system (due to the dots) by removing the underlying anisotropy (the 1D ripple-like structures oriented parallel to the ion beam direction). Thus, our results indicate the possibility of only two surface structures under prolonged simultaneous sputtering and sample rotation at off-normal incidence: dots, and non-oriented cell-like structures. According to a previous study, these dots are restricted to $\mu \gtrsim 3$ and $\sigma \gtrsim 1$. 


\subsection{Outlook}

The main focus of the study has been on $\theta<\theta_{c}$, but $\theta>\theta_{c}$ might yield some interesting results. Expecially, as regards dot formation. For instance, the dots emerge with the ripple-like structures oriented parallel to the ion beam direction. These structures are dominant in the high $\mu$ region, whereas the ripples oriented perpendicular to the ion beam direction are dominant in the low $\mu$ region (for $\theta<\theta_{c}$ ). However, for $\theta>\theta_{c}$ ripple structures, oriented parallel to the ion beam direction, are known to be dominant in the low $\mu$ region. Thus, if the dots always emerge with these structures, of parallel orientation, perhaps they are formed in the low $\mu$ region for $\theta>\theta_{c}$ with or without rotation. Another possibility in further studies, is to use other non-Gaussian energy distributions along the lines of Ref. 43] where an exponential distribution was found to be more realistic at grazing incidence.

Also, we have studied the two unlimited mobility surface diffusion models in the literature. Though not in great detail, nevertheless, we have found the same topographies, while alternating both. We found the presence of Schwoebel barriers to only affect the form of the wavelength-time relationship; i.e, inverse law instead of exponential law at high temperatures. A detailed study of the differences between the two models is promising, expecially to clarify the role of Schwoebel barriers in more detail. In the course of this study, a variation of the power $n$ in the Hamiltonian model (say $n=1$ model) gave some profiles that are different from the ones shown here. Thus one could take a look at this, and try to explain why it is so different from the usual topographies. Moreover, it will be interesting to consider the unlimited mobility models as well.

In the thermally activated Arrhenius surface diffusion model, the effect of Schwoebel barriers can be studied by simply including the relevant term in the energy equation. However, it is not that simple in reality, at least as far as metallic materials are concerned. For instance, on such metallic surfaces, a diffusion bias is often discussed relative to some crystallographic direction. It will be interesting to consider such spatially-directional constraints in further studies; possibly by introducing different energy barriers to step edges along different spatial directions.

Although there are very strong indications that the HKGK model, with a suitable choice of coefficients, might belong to the KS universality class, it will be worth while to derive its continuum representation, either using the master equation approach (along the lines of Ref. [88]), or some other method [1].

In future experiments, a systematic study covering the full parameter space, at least in the range $\sigma \leq \mu$ will be very interesting. So far, to our 
knowledge, this has not been done. Furthermore, one could investigate the small-time behavior, i.e. the ripple formation process, experimentally. In this way one could verify whether the two different creation mechanisms we have observed, hole-coalescence and creation from a rough surface, can be observed in experimental systems.

Finally, smoothening could be included in the sputtering behavior, along the lines of Ref. [114]. This has been discussed in the continuum theory [71, as effective surface relaxation. We have not implemented it explicitly in the sputtering mechanism, because it is implicit in our surface diffusion mechanism, manifested in the higher effective temperature, due to local heating (i.e, in thermal spikes) of the surface at the impact point. 


\section{Bibliography}

[1] A.-L. Barabási and H. E. Stanley, Fractal Concepts in Surface Growth (Cambridge University Press, Cambridge, 1995).

[2] Harold F. Winters and J. W. Coburn, Surf. Sci. Rep. 14, 161 (1992).

[3] Eun-Hee Cirlin, John J. Vajo, and T. C. Hasenberg, J. Vac. Sci. Technol. B 12, 269 (1994).

[4] Elliot A. Eklund, R. Bruinsma, and J. Rudnick, Phys. Rev. Lett. 67, 1759 (1991).

[5] G. Carter, Erosion and Growth of Solids Stimulated by Atom and Ion Beams, edited by G. Kiriakidis, G. Carter, and J. L. Whitton (Martinus Nijhoff, Hingham, MA, 1986), pp 70-79.

[6] J. L. Vossen and W. Kern, Thin-Film Processes (Academic, New York, 1978).

[7] G. Carter, J. Phys. D: Appl. Phys. 34, R1 (2001).

[8] T. M. Mayer, E. Chason, and A. J. Howard, J. Appl. Phys. 76, 1633 (1994).

[9] E. Chason and T. M. Mayer, Appl. Phys. Lett. 62, 363 (1993).

[10] E. Chason, J. Y. Tsao, K. M. Horn, S. T. Picraux, and H. A. Atwater, J. Vac. Sci. Technol. A 8 (3), 2507 (1990).

[11] E. Chason, T. M. Mayer, B. K. Kellerman, D. T. McIlroy, and A. J. Howard, Phys. Rev. Lett. 72, 3040 (1994).

[12] S. Habenicht, W. Bolse, K. P. Lieb, K. Reimann, and U. Geyer, Phys. Rev. B 60, R2200 (1999).

[13] K. Elst, W. Vandervorst, J. Alay, J. Snauwaert, and L. Hellemans, J. Vac. Sci. Technol. B 11, 1968 (1993).

[14] G. Carter and V. Vishnyakov, Phys. Rev. B 54, 17647 (1996).

[15] Z. X. Jiang and P. F. A. Alkemade, Appl. Phys. Lett. 73, 315 (1998). 
[16] J. Erlebacher, M. J. Aziz, E. Chason, M. B. Sinclair, and J. A. Floro, Phys. Rev. Lett. 82, 2330 (1999).

[17] T. K. Chini, M. K. Sanyal, and S. R. Bhattacharyya, Phys. Rev. B 66, 153404 (2002).

[18] J. Kim, D. G. Cahill, and R. S. Averback, Phys. Rev. B 67, 045404 (2003).

[19] T. K. Chini, F. Okuyama, M. Tanemura, and K. Nordlund, Phys. Rev. B 67, 205403 (2003).

[20] S. W. MacLaren, J. E. Baker, N. L. Finnegan, and C. M. Loxton, J. Vac. Sci. Technol. A 10, 468(1992).

[21] G. Carter, V. Vishnyakov, Yu. V. Martynenko, and M. J. Nobes, J. Appl. Phys. 78, 3559 (1995).

[22] S. Habenicht, K. P. Lieb, J. Koch, and A. D. Wieck, Phys. Rev. B 65, 115327 (2002).

[23] S. Jay Chey, Joseph E. Van Nostrand, and David G. Cahill, Phys. Rev. B 52, 16696 (1995).

[24] S. Jay Chey, Joseph E. Van Nostrand, and David G. Cahill, Phys. Rev. Lett. 76, 3995(1996).

[25] S. Facsko, T. Dekorsy, C. Koerdt, C. Trappe, H. Kurz, A. Vogt, and H. L. Hartnagel, Science 285, 1551(1999).

[26] R. Gago, L. Vázquez, R. Cuerno, M. Varela, C. Ballesteros, and J. M. Albella, Appl. Phys. Lett. 78, 3316 (2001).

[27] S. Facsko, H. Kurz, and T. Dekorsy, Phys. Rev. B 63, 165329 (2001).

[28] A. Zalar, Thin Solid Films 124, 223 (1985).

[29] E. -H. Cirlin, J. J. Vajo, R. E. Doty, and T. C. Hasenberg, J. Vac. Sci. Technol. A 9, 1395 (1991).

[30] F. Frost, A. Schindler, and F. Bigl, Phys. Rev. Lett. 85, 4116 (2000).

[31] M. Ritter, M. Stindtmann, M. Farle, and K. Baberschke, Surf.Sci. 348, 243 (1996). 
[32] S. Rusponi, G. Costantini, C. Boragno, and U. Valbusa, Phys. Rev. Lett. 81, 2735 (1998); and references therein.

[33] G. Costantini, F. Buatier de Mongeot, C. Boragno, and U. Valbusa, Phys. Rev. Lett. 86, 838 (2001).

[34] Thomas Michely and George Comsa, Nucl. Instrum. Methods Phys. Res. Sect. B 82, 207 (1993).

[35] M. V. Ramana Murty, T. Curcic, A. Judy, B. H. Cooper, A. R. Woll, J. D. Brock, S. Kycia, and R. L. Headrick, Phys. Rev. Lett. 80, 4713 (1998); M. V. Ramana Murty, T. Curcic, A. Judy, B. H. Cooper, A. R. Woll, J. D. Brock, S. Kycia, and R. L. Headrick, Phys. Rev. B 60, 16956 (1999).

[36] J. Naumann, J. Osing, A. J. Quinn, and I. V. Shvets, Surf. Sci. 388, 212 (1997).

[37] S. Rusponi, C. Boragno, and U. Valbusa, Phys. Rev. Lett. 78, 2795 (1997).

[38] S. Rusponi, G. Costantini, C. Boragno, and U. Valbusa, Phys. Rev. Lett. 81, 4184 (1998); and references therein.

[39] M. A. Makeev, R. Cuerno, and A.-L. Barabási, Nucl. Instr. and Meth. in Phys. Res. B 197, 185 (2002).

[40] P. Sigmund, Phys. Rev. 184, 383 (1969).

[41] R. Mark Bradley and J. M. E. Harper, J. Vac. Sci. Technol. A 6, 2390 (1988).

[42] R. Cuerno and A.-L. Barabási, Phys. Rev. Lett. 74, 4746 (1995).

[43] M. Feix, A. Hartmann, R. Kree, J. Muñoz-García, and R. Cuerno, Phys. Rev. B 71, 125407 (2005).

[44] K. Wittmaack, J. Vac. Sci. Technol. A 8, 2246 (1990).

[45] J. Erlebacher, M. J. Aziz, E. Chason, M. B. Sinclair, and J. A. Floro, J. Vac. Sci. Technol. A 18, 115 (2000).

[46] J. J. Vajo, R. E. Doty, and E.-H. Cirlin, J. Vac. Sci. Technol. A 6, 76 (1988).

[47] H.-N. Yang, G.-C. Wang, and T.-M. Lu, Phys. Rev. B 50, 7635 (1994). 
[48] G. Carter, Phys. Rev. B 59, 1669 (1999).

[49] M. Kardar, G. Parisi, and Y.-C. Zhang, Phys. Rev. Lett. 56, 889 (1986).

[50] R. Mark Bradley, Phys. Rev. E 54, 6149 (1996).

[51] J. Krug and H. Spohn, Phys. Rev. A 38, 4271 (1988).

[52] Y. Kuramoto and T. Tsuzuki, Prog. Theor. Phys. 55, 356 (1976); G. I. Sivashinsky, Acta Astronaut. 4, 1177 (1976); Y. Kuramoto, Chemical Oscillations, Waves, and Turbulence (Springer, Berlin, 1984); G. I. Sivashinsky, Acta Astronaut. 4, 1177 (1977); G. I. Sivashinsky and D. M. Michelson, Prog. Theor. Phys. 63, 2112 (1980).

[53] B. Nicolaenko, Physica D 20, 109 (1986).

[54] V. Yakhot, Phys. Rev. A 24, 642 (1981).

[55] S. Zaleski, Physica D 34, 427 (1989).

[56] K. Sneppen, J. Krug, M. H. Jensen, C. Jayaprakash, and T. Bohr, Phys. Rev. A 46, R7351 (1992).

[57] F. Hayot, C. Jayaprakash, and Ch. Josserand, Phys. Rev. E 47, 911 (1993).

[58] C. Jayaprakash, F. Hayot, and R. Pandit, Phys. Rev. Lett. 71, 12 (1993).

[59] V. S. L'vov and I. Procaccia, Phys. Rev. Lett. 69, 3543 (1992).

[60] I. Procaccia, M. H. Jensen, V. S. L'vov, K. Sneppen, and R. Zeitak, Phys. Rev. A 46, 3220 (1992); V. S. L'vov and I. Procaccia, Phys. Rev. Lett. 72, 307 (1994); C. Jayaprakash, F. Hayot, and R. Pandit, ibid. 72, 308 (1994).

[61] R. Cuerno and K. B. Lauritsen, Phys. Rev. E 52, 4853 (1995).

[62] D. E. Wolf, Phys. Rev. Lett. 67, 1783 (1991).

[63] R. Bruinsma, Surface Disordering: Growth, Roughening, and Phase Transitions, eds. R. Jullien et al. (Nova Science, New York, 1992).

[64] E. S. Tok, S. W. Ong, and H. C. Kang, Phys. Rev. E 70, 011604 (2004).

[65] J. T. Drotar, Y. P. Zhao, T. M. Lu, and G. C. Wang, Phys. Rev. E 59, 177 (1999). 
[66] S. Park, B. Kahng, H. Jeong, and A.-L. Barabási, Phys. Rev. Lett. 83, 3486 (1999).

[67] M. Rost and J. Krug, Phys. Rev. Lett. 75, 3894 (1995).

[68] J. Muñoz-García, M. Castro, and R. Cuerno, Phys. Rev. Lett. 96, 086101 (2006).

[69] S. Rusponi, G. Costantini, F. B. de Mongeot, C. Boragno, and U. Valbusa, Appl. Phys. Lett. 75, 3318 (1999).

[70] B. Kahng, H. Jeong, and A.-L. Barabási, Appl. Phys. Lett. 78, 805 (2001).

[71] M. A. Makeev and A.-L. Barabasi, Appl. Phys. Lett. 71, 2800 (1997).

[72] E. -H. Cirlin, Thin Solid Films 220, 197 (1992).

[73] R. Mark Bradley and E. -H. Cirlin, Appl. Phys. Lett. 68, 3722 (1996).

[74] F. Frost, Appl. Phys. A 74, 131 (2002).

[75] K.M. Beardmore, N. Grønbech-Jensen, Phys. Rev. E 57, 7278, 1998.

[76] A. Kobayashi and S. Das Sarma, Phys. Rev. B 37, 1039 (1988).

[77] J. M. Thijssen, Computational Physics, (Cambridge University Press, Cambridge, 1999).

[78] D. Marx and J. Hutter, Ab Initio Molecular Dynamics: Theory and Implementation, in Modern Methods and Algorithms of Quantum Chemistry (p. 301-449), Editor: J. Grotendorst, (NIC, FZ Jlich 2000).

[79] E. M. Bringa, R. E. Johnson, and R. M. Papaléo, Phys. Rev. B 65, 094113 (2002).

[80] I. Koponen, M. Hautala, and O.-P. Sievänen, Phys. Rev. B, 54, 13502 (1996).

[81] Details are provided in Table 1 of Ref. [80].

[82] A. K. Hartmann, R. Kree, U. Geyer, and M. Kölbel, Phys. Rev. B 65, 193403 (2002).

[83] M. Stepanova and S. K. Dew, Appl. Phys. Lett. 84, 1374 (2004). 
[84] J. F. Ziegler, J. P. Biersack, and K. Littmark, The Stopping and Range of Ions in Matter (SRIM), (Pergamon, New York 1985); see also http://www.srim.org/.

[85] A.-D. Brown and J. Erlebacher, Phys. Rev. B 72, 075350 (2005).

[86] R. Cuerno, H. A. Makse, S. Tomassone, S. T. Harrington, and H. E. Stanley, Phys. Rev. Lett. 75, 4464 (1995).

[87] G. Carter, B. Navinšek, and J. L. Whitton, in Sputtering by Particle Bombardment, edited by R. Behrisch (Springer-Verlag, Heidelberg, 1983), Vol II.

[88] K. B. Lauritsen, R. Cuerno, and H. A. Makse, Phys. Rev. E 54, 3577 (1996).

[89] J. Krug, M. Plischke, and M. Siegert, Phys. Rev. Lett. 70, 3271 (1993).

[90] J. M. Kim and S. Das Sarma, Phys. Rev. Lett. 72, 2903 (1994).

[91] D. Wolf and J. Villain, Europhys. Lett. 13, 389 (1990).

[92] S. Das Sarma and P. I. Tamborenea, Phys. Rev. Lett. 66, 325 (1991);

P. I. Tamborenea and S. Das Sarma, Phys. Rev. E 48, 2575 (1993).

[93] P. Punyindu Chatraphorn, Z. Toroczkai, and S. Das Sarma, Phys. Rev. B 64, 205407 (2001).

[94] J. Kertësz and D. E. Wolf, J. Phys. A 21, 747 (1988); D. E. Wolf and J. Kertësz, Europhys. Lett. 4, 651 (1987).

[95] C. Dasgupta, S. Das Sarma, and J. M. Kim, Phys. Rev. E 54, R4552 (1996); C. Dasgupta, J. M. Kim, M. Dutta, and S. Das Sarma, ibid. 55, 2235 (1997).

[96] F. Family, J. Phys. A 19, L441 (1986).

[97] S. F. Edwards and D. R. Wilkinson, Proc. R. Soc. London, Ser. A 381, 17 (1982).

[98] Z.-W. Lai and S. Das Sarma, Phys. Rev. Lett. 66, 2348 (1991).

[99] M. Plischke, J. D. Shore, M. Schroeder, M. Siegert, and D. E. Wolf, Phys. Rev. Lett. 71, 2509 (1993).

[100] S. Das Sarma and S. V. Ghaisas, Phys. Rev. Lett. 71, 2510(1993). 
[101] Y. W. Mo, J. Kleiner, M. B. Webb, and M. G. Lagally, Phys. Rev. Lett. 66, 1998 (1991); E. Kopatzki, S. Günther, W. Nichtl-Pecher, and R. J. Brehm, ibid. 284, 154 (1993).

[102] M. Siegert and M. Plischke, Phys. Rev. Lett. 73, 1517 (1994).

[103] For the general form of the hamiltonian $H$ as well as the phase transition that may occur due to the role of its coefficients, see: M. Siegert and M. Plischke, Phys. Rev. Lett. 68, 2035 (1992).

[104] P. Smilauer, M. R. Wilby, and D. D. Vvedensky, Phys. Rev. B 47, 4119 (1993).

[105] E. O. Yewande, A. K. Hartmann, and R. Kree, Phys. Rev. B 71, 195405 (2005).

[106] E. O. Yewande, R. Kree, and A. K. Hartmann, Phys. Rev. B 73, 115434 (2006).

[107] T. Shitara, D. D. Vvedensky, M. R. Wilby, J. Zhang, J. H. Neave, and B. A. Joyce, Phys. Rev. B 46, 6815 (1992).

[108] J. Melngailis, J. Vac. Sci. Technol. B 5, 469 (1987).

[109] W. Primak, Phys. Rev. 98, 1854 (1955).

[110] N.A. Marks, Phys. Rev. B 56, 2441 (1997).

[111] D. Saada, J. Adler, and R. Kalish Phys. Rev. B 59, 6650 (1999).

[112] J. G. Amar and F. Family, Phys. Rev. A 41, 3399 (1990).

[113] Ari-David Brown, Jonah Erlebacher, Wai-Lun Chan and Eric Chason, Phys. Rev. Lett. 95, 056101 (2005).

[114] P. Bedrossian, J. E. Houston, J. Y. Tsao, E. Chason, and S. T. Picraux, Phys. Rev. Lett. 67, 124 (1991). 
Emmanuel O. Yewande

Von-Ossietzky Straße 44

D-37085 Göttingen

Geburtsdatum: $\quad$ 19. Juli 1971

Geburtsort: Ibadan/Nigeria

Staatsangehörigkeit: Nigerianisch

\section{Bildungsgang}

03/1994 B.Sc. in Physik an der Universität Ibadan, Nigeria

06/1999 M.Sc. in Physik an der Universität Ibadan, Nigeria

08/2002 Diplom in verkürzte Angelegenheit Theorie, Abdus Salam International Centre for Theoretical Physics, Trieste, Italy

05/2006 Promotion in Physik an der Universität Göttingen

\section{Berufserfahrung}

11/1995-08/2001

Lehrer (Gymnasium), Oyo State teaching service commission, Nigeria

\section{Auszeichnung}

05/2001 Scholarship of the ICTP for the diploma program

\section{Publikationen}

- E. O. Yewande, R. Kree, and A. K. Hartmann, Physical Review B 73, 115434 (2006).

- E. O. Yewande, A. K. Hartmann, and R. Kree, Physical Review B 71, 195405 (2005).

- E. O. Yewande, Y. Moreno, F. Kun, R. C. Hidalgo, and H. J. Herrmann, Physical Review E 68, 026116 (2003).

\section{Vortragen}

- DPG, Berlin, 2005. Titel: Topography of simultaneously sputtered and rotated solid surfaces: a numerical study

- DPG, Regensburg, 2004. Titel: Non-linear effects in a simulation model of sputter-erosion

Göttingen, 22. März 2006. 